\title{
Spannungsinduzierte Wellenbildung in laserdeponierten Polymer/Metall-Systemen
}

\author{
Dissertation
}

zur Erlangung des mathematisch-naturwissenschaftlichen Doktorgrades

\author{
„Doctor rerum naturalium“ \\ der Georg-August-Universität Göttingen
}

im Promotionsprogramm ProPhys

der Georg-August University School of Science (GAUSS)

vorgelegt von

\section{Susanne Schlenkrich}

geb. Seyffarth

aus Salzgitter

Göttingen, 2014 


\section{Betreuungsauschuss}

Prof. Dr. Hans-Ulrich Krebs,

Institut für Materialphysik, Georg-August-Universität Göttingen

Prof. Dr. Konrad Samwer,

I. Physikalisches Institut, Georg-August-Universität Göttingen

\section{Mitglieder der Prüfungskommission}

Referent: Prof. Dr. Hans-Ulrich Krebs,

Institut für Materialphysik, Georg-August-Universität Göttingen

Korreferent: Prof. Dr. Konrad Samwer

I. Physikalisches Institut, Georg-August-Universität Göttingen

\section{Weitere Mitglieder der Prüfungskommission}

Prof. Dr. Vasily Moshnyaga,

I. Physikalisches Institut, Georg-August-Universität Göttingen

Prof. Dr. Michael Seibt,

IV. Physikalisches Institut, Georg-August-Universität Göttingen

Prof. Dr. Cynthia Volkert,

Institut für Materialphysik, Georg-August-Universität Göttingen

Prof. Dr. Claus Ropers,

IV. Physikalisches Institut, Georg-August-Universität Göttingen

Tag der mündlichen Prüfung: 10. Juni 2014 


\section{Inhaltsverzeichnis}

1 Einleitung 1

2 Theoretischer Hintergrund 5

3 Experimentelle Methoden $\quad 13$

3.1 Gepulste Laserdeposition (PLD) . . . . . . . . . . . . . . . . . . . 13

3.2 Rasterkraftmikroskop $(\mathrm{AFM}) \ldots \ldots \ldots \ldots \ldots$

3.3 Rasterelektronenmikroskop (REM) . . . . . . . . . . . . . . . 16

3.4 Transmissionselektronenmikroskopie (TEM) . . . . . . . . . . . . . 17

3.5 Fokussierte Ionenstrahltechnik (FIB) . . . . . . . . . . . . . . . . . 17

3.6 Größenausschluss-Chromatographie (SEC) . . . . . . . . . . . . . . . . 18

3.7 Röntgennahkantenabsorptionsspektroskopie (NEXAFS) . . . . . . . . . 19

3.8 Profilometrie . . . . . . . . . . . . . . . . . . . . . . . . . . 19

3.9 Indenter . . . . . . . . . . . . . . . . . . . . . . . . . . . . . . . 20

4 Eigenschaften verwendeter Polymere $\quad 21$

4.1 Eigenschaften von Polymethylmethacrylat (PMMA) . . . . . . . . . . . 21

4.2 Eigenschaften von Bisphenol-A-Dimethacrylat (BisDMA) und Polycarbonat $(\mathrm{PC}) \ldots \ldots \ldots \ldots \ldots . \ldots \ldots \ldots$

4.3 Eigenschaften gemischter Polymerschichten . . . . . . . . . . . . 34

5 Spannungsinduzierte Wellenbildung im Polymer/Metall-System 39

5.1 Variation der Cu-Schichtdicke . . . . . . . . . . . . . . . . . . . . . 39

5.2 Variation der PMMA-Schichtdicke . . . . . . . . . . . . . . . . 49

5.3 Einfluss einer Ar-Atmosphäre . . . . . . . . . . . . . . . . . . . 55

5.4 Ausblick und Anwendungen . . . . . . . . . . . . . . . . . 58

5.4 .1 SIEBIMM-Methode . . . . . . . . . . . . . 58

5.4.2 Anordnen der Wellen . . . . . . . . . . . . . . . 61

6 Strategien zum Erreichen glatter Polymer/Metall-Schichtpakete $\quad 69$

6.1 Beeinflussung des Elastizitätsmoduls der Polymerschicht . . . . . . . . 69

6.1.1 Beeinflussung des Elastizitätsmoduls durch UV-Bestrahlung . . 69

6.1.2 Beeinflussung des Elastizitätsmoduls durch thermische Behandlung 74

6.1.3 Beeinflussung des Elastizitätsmoduls durch Verwendung eines Vernetzers . . . . . . . . . . . . . . . . . . . 79 
6.2 Multischichtsysteme . . . . . . . . . . . . . . . . 83

6.2.1 Optimierung zur Herstellung periodischer Polymer/MetallSchichtpakete ................... . 83

6.2.2 PMMA/Cu-Multischichtsysteme . . . . . . . . . . . . . . 84

6.2.3 BisDMA/Cu-Multischichtsysteme . . . . . . . . . . . . 86

6.2.4 $\mathrm{PC} / \mathrm{Cu}-\mathrm{Multischichtsysteme} \mathrm{\ldots ...........} \mathrm{.} \mathrm{.} \mathrm{.} 89$

$\begin{array}{lll}7 & \text { Zusammenfassung } & 95\end{array}$

$\begin{array}{ll}\text { Literaturverzeichnis } & 105\end{array}$ 


\section{Einleitung}

Die Ausbildung von Wellen oder Falten ist ein Phänomen, welches uns im Alltag auf verschiedenste Weisen begegnet. So bildet zum Beispiel die menschliche Haut Falten, wenn sie im Alter an Spannkraft verliert [1,2]. Durch einen ganz anderen Prozess zeigen Gesteinsformationen Wellen an der Oberfläche [3]. Dabei lassen sich wellenartige Gebilde mit Wellenlängen von einigen Kilometern, z. B. Wasserwellen, Sanddünen, Gebirge beobachten, als auch mit wenigen Millimetern, z. B. Hautfalten oder die Rillen eines Fingerabdrucks. In noch kleineren Dimensionen von wenigen Mikro- oder sogar Nanometern lassen sich Wellen auf dünnen Schichten beobachten, die teilweise durch Selbstorganisation oder durch Strukturierung hergestellt werden können.

Die Erzeugung von Wellen auf einer Oberfläche durch den Beschuss von ArIonen wurde bereits im Jahre 1960 von Cunningham et al. untersucht [4]. Dabei lassen sich unterschiedliche wellenförmige Strukturen erzeugen, welche abhängig vom Auftreffwinkel, der Ionenenergie und der Bestrahldauer des eingestrahlten Ionenstrahls sind $[5,6]$. Die Entstehung der wellenartigen Strukturen kann dabei auf ein Wechselspiel zwischen Sputtererosion, Oberflächendiffusion und plastisches Fließen zurückgeführt werden $[7,8]$.

Wellenstrukturen lassen sich auch durch die Deposition einer dünnen Schicht auf einer weichen Unterlage erzeugen, indem man diese Druckspannungen aussetzt. In der Literatur wurde diese Art der Wellenbildung erstmals Anfang der 80-er Jahre von Martin, anhand von dünnen Metallschichten auf einem Poly(dimethylsiloxan)Substrat (PDMS), untersucht [9]. Hierbei wurde das PDMS-Substrat vor der MetallBeschichtung aufgeheizt, so dass sich beim Abkühlen die Schichten zusammenziehen und es aufgrund guter Haftung untereinander zur Ausbildung von Wellen auf der Oberfläche kommt. Dieses Verfahren hat sich seitdem bei Probensystemen bestehend aus einer Polymer- und Metallschicht in der Literatur zur Erzeugung von Wellen etabliert [10-12]. Darüber hinaus können die Spannungen, welche für die Ausbildung der Wellen verantwortlich sind, auch durch die Verwendung einer Zugapparatur 
erzeugt werden [13-15]. Hierbei konnte die Gruppe um Stafford et al. zeigen, dass die ausgebildete Wellenlänge materialspezifisch ist und von den Elastizitätsmoduln der beiden Komponenten abhängt $[13,16]$. Zudem ist es möglich die Wellen gezielt anzuordnen, wobei dies zum einen durch ein Vorstrukturieren des Substrates und zum anderen durch das Anlegen eines anisotropen Spannungsfeldes erzielt werden kann $[11,16]$.

Diese welligen Oberflächen besitzen eine Vielzahl an Anwendungen. So konnte die Gruppe um Fery et al. zeigen, dass sich Kolloidteilchen gezielt durch die Wellenstrukturen anordnen lassen $[17,18]$. Auch zeigen die Wellen eine Art Lotus-Effekt, indem sie die Benetzbarkeit von Flüssigkeiten stark beeinflussen [19]. Desweiteren lassen sich beliebige Formen und Muster auf Oberflächen erzeugen, was durch das gezielte Schwellen des Polymers in Bereichen, welche mittels AFM strukturiert wurden, hervorgerufen wird [20].

Zur Herstellung von Polymer/Metall-Schichtsystemen eignet sich die gepulste Laserdeposition besonders gut, da mit diesem Verfahren ein breites Spektrum an Materialklassen deponiert werden kann [21,22]. Dabei zeigte sich in ersten Experimenten, dass es bei der Deposition einer dünnen Metallschicht auf ein Polymer zur Ausbildung großer Wellen mit Wellenlängen von einigen Mikrometern kommt. Dies ist beispielhaft in einer REM-Querschnittsaufnahme für ein PMMA(450 nm)/Cu(50 nm)-Schichtpaket mit 11 Doppelschichten in Abbildung 1.1 dargestellt.

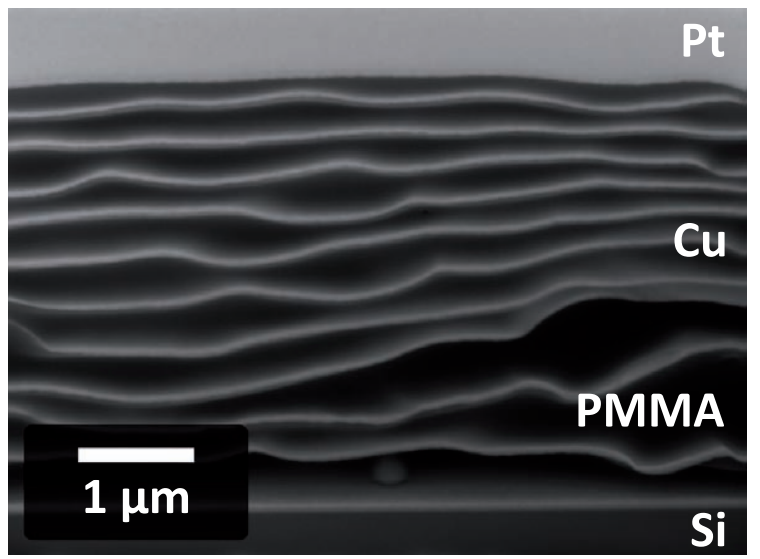

Abbildung 1.1: REM-Querschnittsaufnahme eines PMMA(450 nm)/Cu(50 nm)-Schichtpakets mit 11 Doppelschichten. Das PMMA/Cu-Schichtpaket zeigt eine starke Wellenbildung, die auf die ablationsbedingten hohen Spannungen innerhalb der Metallschicht (bei der gepulsten Laserdeposition) zurückzuführen sind. Die laserdeponierten PMMA-Schichten besitzen eine geringe Härte, wodurch sie sehr weich sind und können somit die hohen Spannungen nicht kompensieren, was zu der zu untersuchenden Wellenbildung führt. 
Die Ausbildung der Wellen liegt zum einen an der weichen PMMA-Schicht und zum anderen an der verspannten Metallschicht. Die laserdeponierten PMMA-Schichten besitzen aufgrund des Ablationsprozesses eine stark verkürzte Kettenlänge, wodurch die Schichten sehr weich sind [23]. Zusätzlich weisen laserdeponierte Metallschichten starke Verspannungen aufgrund der hohen kinetischen Energien der deponierten Teilchen auf [24]. Einerseits werden die hochenergetischen Ionen (ca. $100 \mathrm{eV}$ ) in die PMMASchicht implantiert und andererseits kommt es zu einem Verdichten der gewachsenen Metallschicht („shot peening“) [25-27]. Da die PMMA-Schicht auf dem Substrat eine gute Haftung aufweist und keine Ausdehnung zulässt, können die Spannungen nur durch die Ausbildung von Wellen an der Oberfläche kompensiert werden.

Das Ziel dieser Arbeit ist es, die spannungsinduzierte Wellenbildung in laserdeponierten Polymer/Metall-Systemen systematisch zu untersuchen und zu verstehen.

Dabei soll zum einen studiert werden, von welchen Parametern, wie z.B. der Metallund der Polymer-Schichtdicke, die Wellenlänge abhängt, und ob es möglich ist, die Wellen gezielt anzuordnen. Desweiteren soll untersucht werden, inwiefern die Beziehung zwischen der Wellenlängen und den Elastizitätsmoduln der beiden Komponenten benutzt werden kann, um den Elastizitätsmodul der deponierten Schichten zu bestimmen.

Zum anderen sollen Strategien zum Erreichen glatter, periodischer Polymer/MetallSchichtpakete vorgestellt werden. Hierbei soll gezeigt werden, wie durch eine gezielte Steigerung des Elastizitätsmoduls der Polymerschichten die Möglichkeit besteht glatte Polymer/Metall-Schichtpakete zu deponieren.

Diese Arbeit ist wie folgt gegliedert: In Kapitel 2 werden die Wellen mit Hilfe der Biegebalkentheorie beschrieben. Hierbei wird anhand eines Modells gezeigt, dass sich die Wellenlänge durch die Elastizitätsmoduln beider Materialkomponenten ausdrücken lässt. Anschließend werden die experimentellen Herstellungs- und Untersuchungsmethoden in Kapitel 3 beschrieben. In Kapitel 4 werden die verwendeten Polymere analysiert, insbesondere das unterschiedliche Ablationsverhalten und die mechanischen Eigenschaften werden dabei untersucht. Anschließend wird in Kapitel 5 zum einen gezeigt, welchen Einfluss die Variation sowohl der $\mathrm{Cu}$ - als auch der PMMA-Schichtdicken auf die Ausbildung der Wellen im Polymer/Metall-System haben. Zum anderen sollen mögliche Anwendungen der Proben mit welligen Oberflächen vorgestellt werden. In Kapitel 6 werden Strategien zum Erreichen glatter, periodischer Polymer/MetallSchichtsysteme vorgestellt und anschließend umgesetzt. Eine Zusammenfassung der gewonnenen Ergebnisse dieser Arbeit wird in Kapitel 7 gegeben. 



\section{Theoretischer Hintergrund}

Eine Doppelschicht, in dieser Arbeit bestehend aus einer dicken PMMA-Schicht und einer dünnen $\mathrm{Cu}$-Schicht, hergestellt mit der gepulsten Laserdeposition, zeigt Wellenbildung an der Oberfläche. Aufgrund der hohen kinetischen Energie der ablatierten Teilchen ( $100 \mathrm{eV}$ ) kommt es zur Implantation der Cu-Atome in die PMMASchicht. Dies hat zur Folge, dass aufgrund der Materialverdichtung innerhalb der Schicht kompressive Spannungen entstehen, wodurch es zur Ausbildung der Wellen kommt. Desweiteren bewirkt die Implantation eine gute Haftung der beiden Materialien aneinander, so dass die PMMA-Schicht gezwungen ist, sich genauso zu verformen, wie es die Cu-Schicht vorgibt. Das heißt, es wirken zwei Kräfte in diesem System, zum einen die Kraft $F_{P}$, welche durch die kompressiven Spannungen ausgeübt wird, und zum anderen eine Kraft $F_{Z}$ in z-Richtung, welche aufgrund der Haftung der beiden Materialien erzeugt wird. In Abbildung 2.1 ist die Kräfteverteilung im PMMA/CuSchichtsystem schematisch dargestellt.

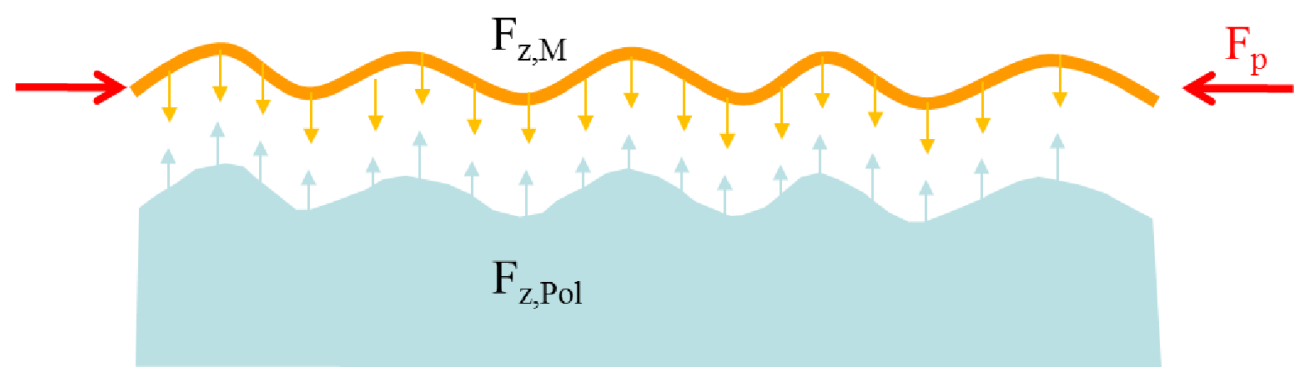

Abbildung 2.1: Schematische Darstellung der Kräfteverteilung in einem PMMA/Cu-Schichtsystem. Durch das Auftreten von Kompressionskräften $F_{P}$ durch Ionenimplantation und Kräften $F_{Z}$ in vertikaler Richtung aufgrund guter Haftungseigenschaften zwischem dem Polymer und dem Metall resultiert die zu untersuchende Wellenbildung.

In der Literatur ist die Wellenbildung zum einen anhand von zwei-dimensionalen Modellen durch die Näherung einer dünnen Platte beschrieben worden [28, 29]. In einer vereinfachten Darstellung lassen sich die Wellen mit Hilfe der Biegebalkentheorie beschreiben, was die Theorie der Wellenbildung auf ein eindimensionales Problem 
reduziert. Dazu betrachtet man einen Balken, der an zwei Punkten gelagert ist. Wirkt auf diesen die Schwerkraft $F_{Z}$, so kommt es zu einem Durchbiegen in z-Richtung (siehe Abbildung 2.2). Die entstandene Kurve wird als Biegelinie $w(x)$ bezeichnet, welche es im Folgenden zu bestimmen gilt. Dafür wird die Breite $b$ des Balkens als sehr klein angenommen. Desweiteren nähert man in einem ersten Schritt, dass der Biegebalken aus mehreren Balkensegmenten besteht. Die Bernoulli-Hypothese besagt, dass die Balkenquerschnitte während der Deformation eben und senkrecht zur verformten Balkenachse bleiben [30].

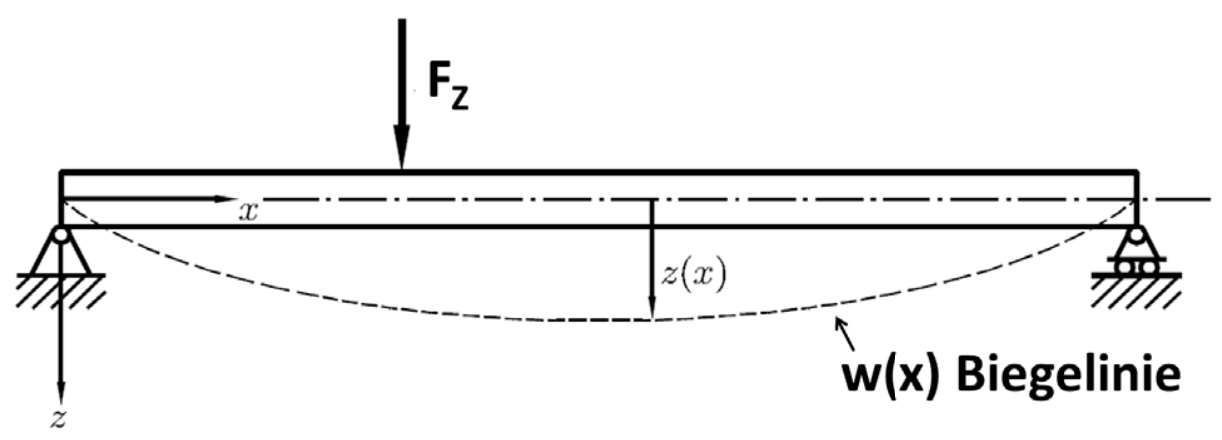

Abbildung 2.2: Mit Hilfe der Biegebalkentheorie soll die Wellenbildung im Polymer/Metall-System näher betrachtet und beschrieben werden. Die Metallschicht soll dazu als ein an zwei Punkten gelagerter Biegebalken angesehen werden (nach [31]).

Betrachtet man nun ein einzelnes Balkensegment in Abbildung 2.3, so wird dieses im oberen Teil gestaucht und im unteren Teil gedehnt. In der Mitte des Balkens gibt es einen Bereich, der den gestauchten Teil von dem gedehnten Teil trennt. Dieser Bereich wird als neutrale Faser bezeichnet und zeichnet sich dadurch aus, dass hier während der gesamten Verformung die gleiche Länge beibehalten wird [32].

In Abbildung 2.3 wird die neutrale Faser durch die gestrichelte Linie dargestellt. Desweiteren bezeichnet $\rho$ den Krümmungsradius, und der Kehrwert von $\rho$ beschreibt die Krümmung, welche näherungsweise der zweiten Ableitung der Biegelinie entspricht, wenn man eine schwache Durchbiegung annimmt.

$$
w(x)=\frac{1}{\rho}
$$

Als nächstes werden die auf den Balken wirkenden Kräfte $F_{Z}$ und $F_{P}$, die im Polymer/Metall-System wesentlich sind, betrachtet. Abbildung 2.4 zeigt beispielhaft welche Auswirkungen die angreifenden Kräfte auf ein Balkensegment haben. 


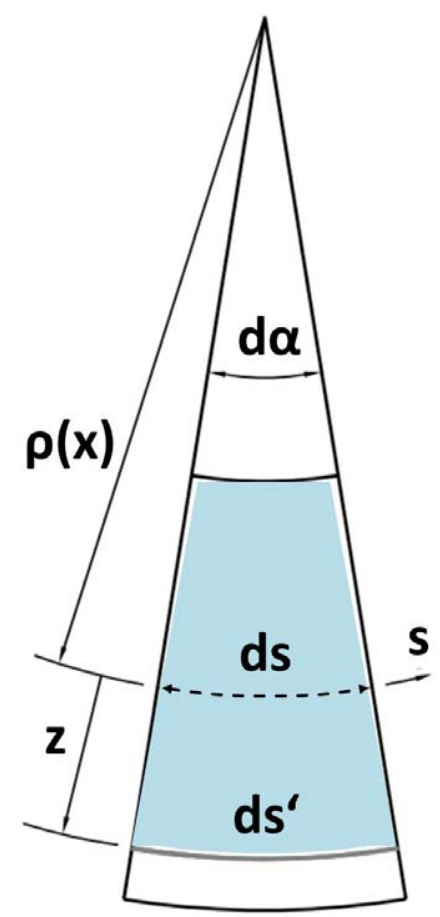

Abbildung 2.3: Teilstück eines Biegebalkens, welcher im oberen Teil gestaucht und im unteren Teil gedehnt wird. Der Bereich, der die Stauchung von der Dehnung trennt, wird neutrale Faser genannt (nach [31]).
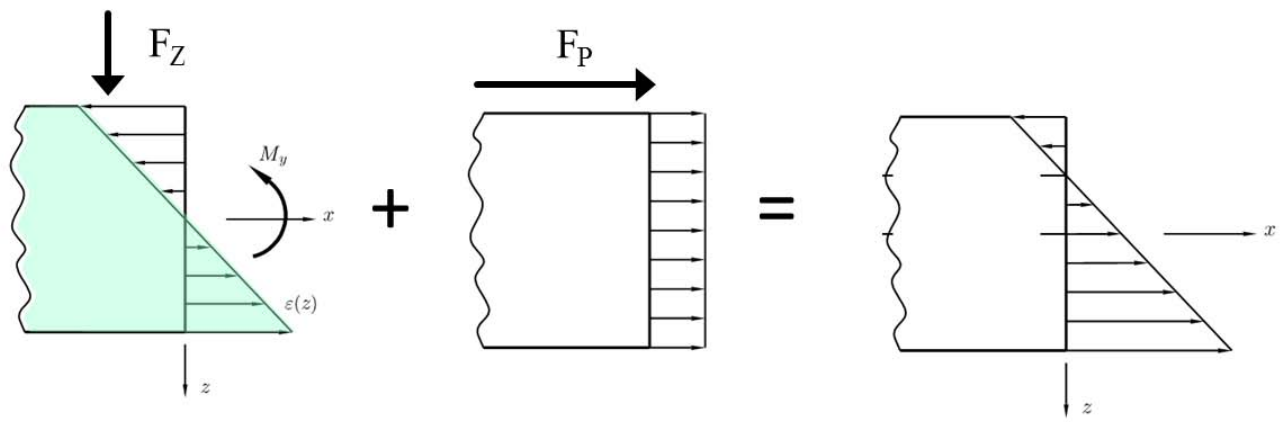

Abbildung 2.4: Schematische Darstellung der Verformung eines Balkensegments durch das Angreifen der beiden Kräfte $F_{Z}$ und $F_{P}$ (nach [31]).

Die Überlagerung der beiden Kräfte $F_{Z}$ und $F_{P}$, durch die einerseits die jeweiligen Bereiche eines Balkensegments gestaucht und gedehnt werden, und andererseits Druckspannungen dafür sorgen, dass das Balkensegment auch in x-Richtung gestreckt wird, führt zu einer Verformung des Balkensegments wie im rechten Bereich der schematischen Darstellung in Abbildung $2.4 \mathrm{zu}$ erkennen ist. Die im Balkensegment 
wirkende Spannung $\sigma$ ist nach Hooke definiert durch die Multiplikation der Dehnung $\epsilon$ mit dem Elastizitätsmodul $E$.

$$
\sigma=E \epsilon
$$

Im Falle der senkrecht angreifenden Kraft $F_{Z}$ ergibt sich für die Biegespannung $\sigma(z)$ folgender Ausdruck:

$$
\sigma(z)=\frac{M_{y}}{I_{y}} z \quad \text { mit } \quad I_{y}=\frac{h^{3} b}{12}
$$

Hierbei ist $M_{y}$ das Biegemoment und $I_{y}$ das Flächenträgheitsmoment (für ein Rechteck mit der Höhe $h$ und der Breite $b$ ). Für das Biegemoment, welches definiert ist aus der Kraft mal dem Hebelarm, erhält man:

$$
M_{y}=\frac{E I_{y}}{\rho}=E I_{y} w
$$

Dabei lässt sich das Biegemoment auch durch die zweite Ableitung der Biegelinie ausdrücken, wenn man Gleichung (2.1) hinzuzieht. Für die horizontal angreifende Kraft lässt sich die Spannung durch die anliegende Kraft $F_{P}$ auf die Fläche $A$ des Balkensegments ausdrücken:

$$
\sigma(z)=\frac{F_{P}}{A}
$$

Für das dazugehörige Biegemoment ergibt sich:

$$
M_{P}=\sigma_{P} w
$$

Aus Gleichung (2.3) und (2.5) erhält man aufgrund der Überlagerung der zwei angreifenden Kräfte nun folgende Gleichung für die Biegespannung:

$$
\sigma(z)=\frac{F_{P}}{A}+\frac{M_{y}}{I_{y}} z
$$

Für das Biegemoment der zwei angreifenden Kräfte unter Verwendung von Gleichung (2.4) und (2.6) ergibt sich: 


$$
M=\sigma_{P} w+E I_{y} w
$$

Diese Differentialgleichung der Biegelinie, welche das Verhalten der Biegelinie in Abhängigkeit der zwei angreifenden Kräfte $F_{Z}$ und $F_{P}$ beschreibt, soll im Folgenden gelöst werden. Dabei werden durch ein- und zweimaliges Ableiten der DGL mit der Querkraft Q und der Streckenlast q neue Parameter eingeführt.

$$
\begin{aligned}
& M=Q=\sigma_{P} w+E I_{y} w \\
& M=q=\sigma_{P} w+E I_{y} w
\end{aligned}
$$

Die Streckenlast $q$ ist dabei definitionsgemäß als eine Kraft pro Wegstrecke zu verstehen und kann auch ausgedrückt werden als:

$$
q=\sigma_{z} b
$$

Dadurch wird aus Gleichung (2.10):

$$
\sigma_{z} b=\sigma_{P} w+E I_{y} w
$$

Zur Lösung der DGL (2.12) soll eine unendlich lange Strebe auf einem elastischem Medium, welches nach unten unendlich ausgedehnt ist, angenommen werden [33]. Die Breite $b$ der Strebe bzw. des Mediums wird als sehr klein angenommen, sodass die Biegespannung in y-Richtung durch $\sigma_{y} \quad 0$ genähert werden kann und die Höhe der Strebe sei $h$. Die Verbiegung der Strebe wird dabei als sinusförmig angenommen, somit ergibt sich für die Auslenkung $w(x)$ folgender Lösungsansatz:

$$
w(x)=w_{m} \sin \frac{\pi x}{l}
$$

Dabei bezeichnet $w_{m}$ die Amplitude der Welle und $l$ die halbe Wellenlänge. Aufgrund der Verbiegung der Strebe muss sich auch das elastische Medium verbiegen, für die Biegespannung gilt: 


$$
\sigma_{z}=2 \pi \frac{w}{l} E_{P}
$$

Hierbei entspricht $E_{P}$ dem Elastizitätsmodul des elastischen Mediums, welches in dem Polymer/Metall-System dem der Polymerschicht entspricht. Unter Verwendung von Gleichung (2.3), wodurch sich das Flächenträgheitsmoment der Strebe auch umschreiben lässt als $I_{y}=\frac{h^{3} b}{12}$ und Auflösen der Gleichung (2.12) nach $\sigma$ erhält man:

$$
\sigma=\frac{E_{M} \pi^{2}}{12}\left(\frac{h_{M}}{l}\right)^{2}+\frac{2 E_{P}}{\pi}\left(\frac{l}{h_{M}}\right)
$$

Hierbei bezeichnet $h_{M}$ die Schichtdicke der Strebe, welches in dem Polymer/MetallSystem der Metallschicht entspricht. Gleichung (2.15) ist in Abbildung 2.5 graphisch dargestellt. Dabei fällt auf, dass die Spannung nur von dem Bruch $\frac{l}{h}$ abhängt, wobei der erste Term der Gleichung den asymptotischen Abfall und der zweite Term die Gerade beschreibt. Die Gleichung wird also insgesamt durch die durchgezogene Linie dargestellt, welche ein Extremum besitzt, welches durch Nullsetzung der 1. Ableitung der Spannung $\sigma$ nach $\frac{l}{h}$ bestimmt werden kann:

$$
\frac{d \sigma}{d\left(\frac{l}{h}\right)}=0
$$

Daraus ergibt sich als kritische Wellenlänge:

$$
\lambda_{k r i t}=2 \pi h_{M}\left(\frac{E_{M}}{3 E_{P}}\right)^{\frac{1}{3}}
$$

und die kritische Spannung ergibt sich zu:

$$
\sigma_{k r i t} \quad 0,548\left(E_{M}\right)^{\frac{1}{3}}\left(E_{P}\right)^{\frac{2}{3}}
$$

Gleichung (2.17) zeigt, dass die Wellenlänge nur von den Elastizitätsmoduln der beiden Komponenten und von der Schichtdicke $h_{M}$ der Metallschicht abhängt. Anhand dieses Zusammenhangs ist es möglich, bei Kenntnis der Wellenlänge und des Elastizitätmoduls einer Komponente, den Elastizitätsmodul der anderen Komponente zu bestimmen. 


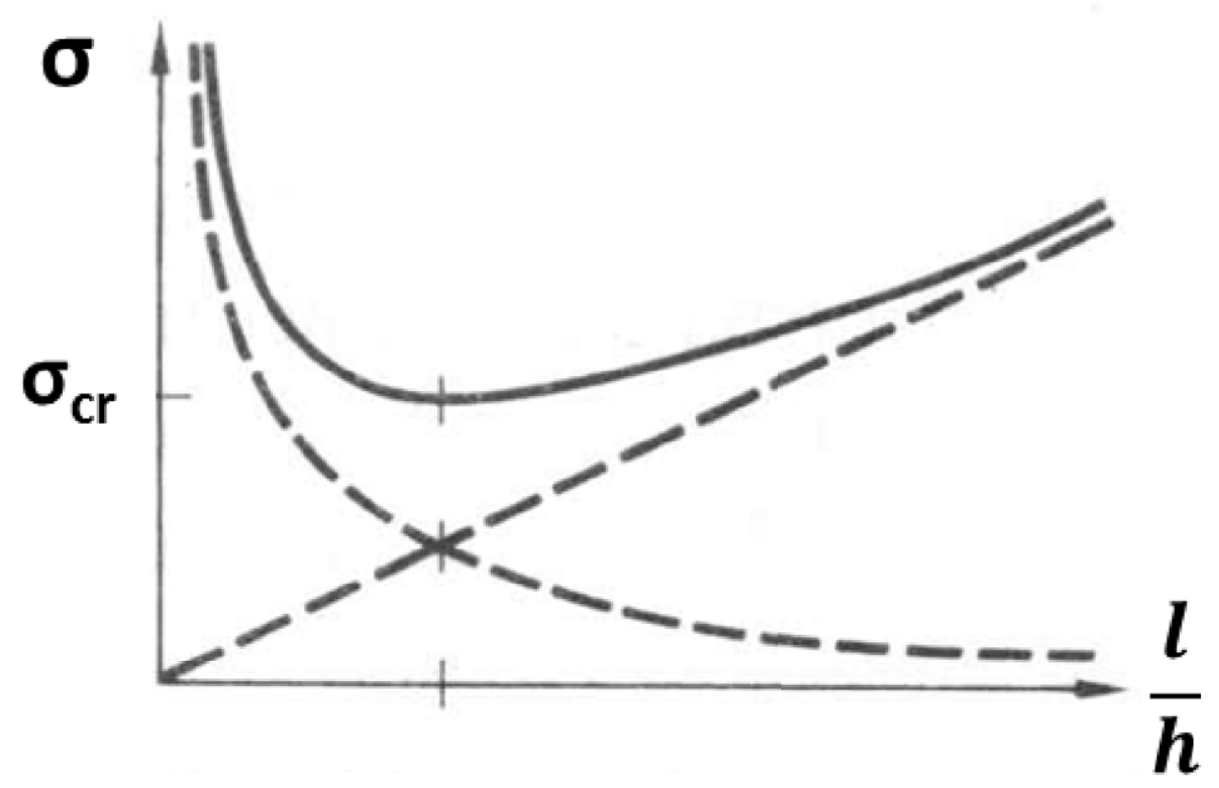

Abbildung 2.5: Graphische Darstellung der Gleichung (2.15). Der Kurvenverlauf der Spannung $\sigma$ in Abhängigkeit vom Verhältnis aus der halben Wellenlänge $l$ und der Schichtdicke $h$ durchläuft ein Minimum (nach [33]).

In der Literatur wird Gleichung (2.17) von vielen Gruppen zur Bestimmung von Elastizitätsmoduln verwendet [11,13,34,35]. Diese Methode wird dabei mit dem Kürzel „SIEBIMM“ (strain-induced elastic buckling instability for mechanical measurements) bezeichnet. Historisch wurden theoretische Studien zur Berechnung von Oberflächenwelligkeiten schon in den 1940-er Jahren an Verbundplatten durchgeführt [33,36,37].

Die Formel muss erweitert werden, wenn das Doppelschichtsystem auf einem starren Untergrund haftet. In dieser Arbeit beispielsweise befindet sich die Polymer/MetallSchicht auf einem Siliziumsubstrat. Dadurch kommt der Schichtdicke der Polymerschicht eine höhere Gewichtung zu, wodurch sich Gleichung (2.14) folgendermaßen verkompliziert:

$$
\sigma_{z}=-\frac{w_{m}}{h_{P}} E_{P} \Theta^{2} f(\Theta) \sin \frac{\pi x}{l}
$$

Hierbei ist $\Theta=\frac{\pi h_{P}}{l}$ mit $h_{P}$ der Schichtdicke der Polymerschicht und $f(\Theta)$ ist mit $\nu_{P}$ als der Poissonzahl der Polymerschicht gegeben durch:

$$
f(\Theta)=\frac{2}{\Theta} \frac{\left(3-\nu_{P}\right) \sinh \Theta \cosh \Theta+\left(1+\nu_{P}\right) \Theta}{\left(\left(1+\nu_{P}\right)\left(3-\nu_{P}\right)^{2} \sinh ^{2} \Theta-\left(1+\nu_{P}\right)^{3} \Theta^{2}\right)}
$$


Durch Berücksichtigung der Polymerschichtdicke erweitert sich Gleichung (2.15) wie folgt:

$$
\sigma=\frac{E_{M}}{12}\left(\frac{h_{M}}{h_{P}}\right)^{2} \Theta^{2}+E_{P}\left(\frac{h_{P}}{h_{M}}\right) f(\Theta)
$$

Diese Gleichung lässt sich nicht mehr so einfach lösen wie zuvor, was zeigt, dass die Berücksichtigung des Substrates die Herleitung eines formellen Zusammenhangs für die Wellenbildung sehr komplex werden lässt. In dieser Arbeit gilt also im folgenden zu berücksichtigen, dass die SIEBIMM-Methode mit der Formel (2.17) nur angewendet werden darf, wenn ein Substrateinfluss auszuschließen ist. 


\section{Experimentelle Methoden}

In diesem Kapitel werden die verwendeten Herstellungs- und Untersuchungsmethoden vorgestellt. Zunächst wird die gepulste Laserdeposition beschrieben. Desweiteren werden die verwendeten Mikroskope zur Untersuchung der Oberflächenmorphologie vorgestellt. Abschließend wird ein Einblick in die fokussierende Ionenstrahltechnik gegeben, mit der Probenquerschnitte erstellt wurden.

\subsection{Gepulste Laserdeposition (PLD)}

In dieser Arbeit wurde zur Probenherstellung die gepulste Laserdeposition (engl. pulsed laser deposition, PLD) verwendet. Dieses Verfahren zeichnet sich insbesondere durch den stöchiometrischen Materialübertrag und sein breites Spektrum an Materialklassen, wie Metalle, Oxide, Keramiken oder Polymere, aus, die als dünne Schichten hergestellt werden $[21,22]$. Desweiteren lassen sich mit Hilfe dieser flexiblen Herstellungsmethode auch komplexe Schichtsysteme aus verschiedenen Materialklassen erzeugen.

Dabei wird ein gepulster Laserstrahl hoher Energie und Intensität auf ein Festkörpertarget, welches sich in einer Ultrahochvakuum (UHV-) Kammer befindet, fokussiert. Dies hat zur Folge, dass sich die Targetoberfläche innerhalb weniger Nanosekunden sehr stark erhitzt ( $6000 \mathrm{~K}$ ) und sich ein quasi-zweidimensionales Plasma ausbildet [25]. Die einfallende Laserstrahlung wird vom Plasma absorbiert, wodurch dieses hochgradig ionisiert wird. Durch Stoßprozesse im Plasma homogenisieren sich die kinetischen Energien und Geschwindigkeitsvektoren der Teilchen, so dass sich als hauptsächliche Expansionsrichtung die Oberflächennormale einstellt. Die Elektronen im Plasma sind aufgrund ihrer geringen Masse viel beweglicher als die Ionen und ziehen somit eine Raumladungszone auf, durch welche die Ionen beschleunigt werden. Die Ionen erreichen dabei eine mittlere kinetische Energie von etwa $100 \mathrm{eV}$ und die neutralen Atome treffen mit etwa $510 \mathrm{eV}$ auf dem gegenüberliegendem Substrat auf. Diese hochenergetischen Ionen haben zur Folge, dass es zum Absputtern oder auch zur 
Implantation in die bereits aufgewachsene Schicht kommen kann [38]. Dadurch hebt sich die Herstellung mittels PLD von anderen Verfahren wie zum Beispiel Sputterdeposition $\left(E_{\text {kin }} \quad 5-10 \mathrm{eV}\right)$ und thermischen Verdampfen $\left(E_{k i n}<1 \mathrm{eV}\right)$ ab.

Desweiteren kommt es bei der Laserablation auch zum Abtrag von makroskopisch flüssigen Tröpfchen (engl. droplets). Diese entstehen durch „hydrodynamisches Sputtern" und den „subsurface heating“- Effekt [39,40]. Hierbei wird beim hydrodynamischen Sputtern davon ausgegangen, dass sich zapfenförmige Strukturen aufgrund des Laserbeschuss ausbilden und diese sich dann im Laufe der Zeit durch den weiteren Beschuss ablösen und in Form von Tropfen auf dem Substrat auftreffen. Der Effekt des subsurface heating beschreibt das Aufreißen der Targetoberfläche in kleine Tröpfchen, da die Temperatur unter der Targetoberfläche deutlich höher ist als an der Oberfläche.

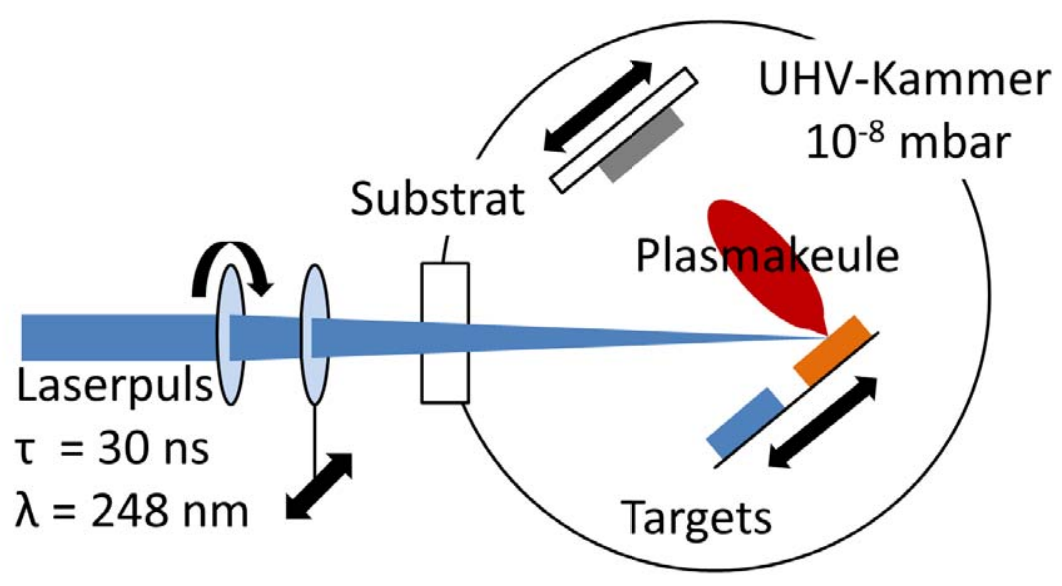

Abbildung 3.1: Schematischer Aufbau der gepulsten Laserdeposition. In einer Ultrahochvakuumkammer trifft ein fokussierter Laserstrahl auf das zu ablatierende Material und verdampft dieses, so dass sich eine Plasmakeule ausbildet, welche senkrecht von der Targetoberfläche expandiert. Auf einem Substrat auf der gegenüberliegenden Seite werden die Atome und Ionen aufgefangen und bilden eine Schicht aus.

In Abbildung 3.1 ist ein schematischer Aufbau der PLD-Anlage dargestellt. Hierbei wurde ein KrF-Excimerlaser LPX 110i der Firma LAMBDA PHYSIK mit einer Wellenlänge von $248 \mathrm{~nm}$ und einer Pulsdauer von $30 \mathrm{~ns}$ verwendet. Die Repetitionsrate für die Metallschichten liegt bei $10 \mathrm{~Hz}$ und für die Polymerschichten bei $5 \mathrm{~Hz}$. Desweiteren befinden sich zwei Linsen im Strahlengang, welche den Strahl auf das Target fokus- 
sieren, um Metallschichten bei einer Energiedichte von ca. $3 \mathrm{~J} / \mathrm{cm}^{2}$ herzustellen. Für Polymerschichten wird eine Linse aus dem Strahlengang automatisch herausgefahren und der Strahl somit defokussiert. Dadurch erhält man einen großen Brennfleck auf dem Target und eine niedrige Energiedichte von $77 \mathrm{~mJ} / \mathrm{cm}^{2}$ wie sie für die Deposition von glatten Polymerschichten notwendig ist. Als Substratmaterial wurden planare Si(111)Substrate verwendet. Als Ausgangsmaterial für die Polymerschichten wurden sowohl PMMA- als auch PC-Platten der Firma Goodfellow verwendet. Als Cu-Target wurden UHV-Dichtungsringe verwendet. Der Target-Substrat-Abstand betrug stets $40 \mathrm{~mm}$ und in der Kammer herrschte ein Basisdruck von $<10^{-8}$ mbar.

\subsection{Rasterkraftmikroskop (AFM)}

Die Rasterkraftmikroskopie eignet sich zur Untersuchung von Oberflächen auf der Nanometerskala. Dieses Verfahren wurde erstmals beschrieben von Binnig [41]. Eine scharfe Messspitze wird zeilenweise in einem definierten Raster über die Probenoberfläche geführt. Hierbei beträgt der Spitzenradius der Nadel $10-20 \mathrm{~nm}$, welche an einer Blattfeder, dem sogenannten Cantilever befestigt ist. Zur Abbildung der

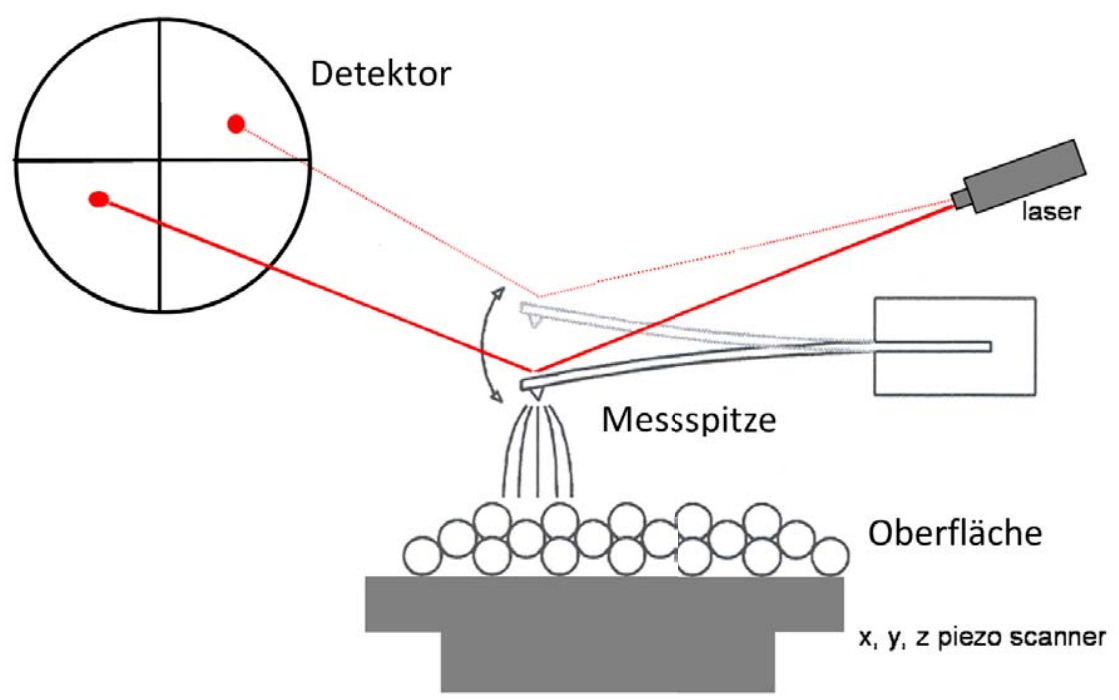

Abbildung 3.2: Schematischer Aufbau eines Rasterkraftmikroskops (Spitze und Cantilever stark verkleinert). 
Probenoberfläche werden die wirkenden Kräfte zwischen der Messspitze und der Probe ausgenutzt. Dies sind zum einen Reibungs- und van der Waals-Kräfte. Um die Probe zerstörungsfrei vermessen zu können, wurde der tapping-mode verwendet. Hierbei wird der Cantilever zu Schwingungen nahe seiner Resonanzfrequenz angeregt, so dass er im unteren Umkehrpunkt die Oberfläche leicht berührt. Aufgrund der Oberflächenstruktur verbiegt sich der Cantilever, welches mit Hilfe von optischen Sensoren gemessen werden kann. Dazu wird ein Laserstrahl auf die spiegelnde Rückseite des Cantilevers gerichtet und die Reflexion von einem Photodetektor aufgefangen. Mit Softwareunterstützung wird dies in eine Oberflächeninformation umgewandelt. Somit erhält man eine dreidimensionale Karte der Oberflächenstruktur. Abbildung 3.2 veranschaulicht die Funktionsweise des Rasterkraftmikroskops.

In dieser Arbeit wurde ein MFP-3D Stand Alone der Firma Asylum Research verwendet. Der maximale Scanbereich betrug $90 \quad 90 \mu^{2}$, und jedes Bild hat eine Auflösung von 512512 Punkten. Es wurden Si-Canitlever der Firma nano and more verwendet. Zur Auswertung der Aufnahmen wurde die Software Gwyddion verwendet.

Desweiteren wurde in Zusammenarbeit mit Matthias Büchsenschütz-Göbeler ein AFM der Firma Bruker vom Typ Icon verwendet. Für die AFM-Indentermessungen wurden Silizium-Cantilever mit einem Spitzenradius von $<10 \mathrm{~nm}$ verwendet mit einem Öffnungswinkel von 20 .

\subsection{Rasterelektronenmikroskop (REM)}

Um die Oberflächenmorphologie über einen größeren Bereich untersuchen zu können wurde ein Rasterelektronenmikroskop verwendet. Hierbei wird die Probenoberfläche von einem fokussierten Elektronenstrahl abgerastert. Dabei wechselwirken Probe und Elektronenstrahl miteinander und es wird ein Spektrum von Elektronen mit unterschiedlichen Energien emittiert [42]. Zur Bilddarstellung werden bevorzugt die Sekundärelektronen aus oberflächennahen Atomschichten detektiert, welche ein tiefenscharfes und kontrastreiches Abbild der Probenoberfläche wiedergeben.

In dieser Arbeit wurden folgende Geräte verwendet. Zum einen entstanden Aufnahmen mit dem im FIB integrieten REM (siehe Kapitel 3.5). Desweiteren wurde ein REM vom Typ Supra 35 der Firma Leo (ehemals Cambridge Instruments) sowie ein REM vom Typ Nova NanoSEM 650 der Firma FEI verwendet. 


\subsection{Transmissionselektronenmikroskopie (TEM)}

Um die Multischichtsysteme in Bezug auf Struktur- und Schichtdickenanalyse untersuchen zu können, wurden mit der fokussierten Ionenstrahltechnik (FIB, siehe Kapitel 3.5) Querschnittslamellen aus den zu untersuchenden Proben präpariert und mit dem Transmissionselektronenmikroskop untersucht. Die Funktionsweise eines TEMs ist vergleichbar mit einem Lichtmikroskop, allerdings erfolgt die Bilderzeugung mit Hilfe hochenergetischer Elektronen [43]. Dabei werden elastisch gestreute Primärelektronen zur Bildgebung detektiert. Dabei kann sowohl ein Realbild als auch ein Beugungsbild der Probe je nach eingestelltem Strahlengang erzeugt werden.

In dieser Arbeit wurde ein TEM vom Typ CM30 der Firma Philips verwendet.

\subsection{Fokussierte lonenstrahltechnik (FIB)}

Die fokussierte Ionenstrahltechnik bietet zum einen die Möglichkeit die Probenoberfläche abzubilden als sie auch gezielt zu bearbeiten. Hierbei stellt das Hauptanwendungsgebiet im Wesentlichen die Materialbearbeitung dar, wodurch sowohl Querschnitte einen Einblick in komplexe Schichtsysteme (wie z. B. Multischichten) geben können, als auch TEM-Lamellen präpariert werden können [44].

Hierbei wird die Probenoberfläche mit schweren Ionen abgerastert, wobei es zum Materialabtrag („Sputtern“) kommt, welches eine gezielte Strukturierung der Probenoberfläche ermöglicht. Desweiteren wechselwirken die schweren Ionen mit der Probe wodurch es auch zur Emission von Elektronen, zum Aufheizen der Probe und zur Implantation von Ionen kommt. Um die Probenoberfläche während der Strukturierung beobachten zu können, befindet sich zusätzlich noch ein Rasterelektronenmikroskop (REM) im Gerät integriert (sog. „dual-beam“-FIB).

In dieser Arbeit wurde ein Zweistrahl-Gerät vom Typ Nova 600 NanoLab der Firma FEI verwendet, welches eine flüssige $\mathrm{Ga}^{+}$-Ionenquelle, ein integriertes REM, einen 5-Achsen Probentisch und einen 3-Achsen-Mikromanipulator (Omniprobe) besitzt. Dabei befindet sich die Elektronenquelle in einem Winkel von 52 zur Ionenquelle ausgerichtet. Hierbei kann eine Probenstelle unabhängig vom Strahlwinkel gleichzeitig von beiden Strahlen bearbeitet werden, wenn sich die Probe in der euzentrischen Höhe befindet. Dabei wird im ersten Schritt die zu untersuchende Probenstelle mit Hilfe des Elektronenstrahls ausgesucht und anschließend mit dem Ionenstrahl bearbeitet. Über 
die Wahl der Beschleunigungsspannung und der Stromstärke des Ionenstrahls kann die Menge des Materialabtrags gezielt eingestellt werden, allerdings hängt dies auch vom Probenmaterial, der Kristallsturktur- und Orientierung, sowie der Oberflächengeometrie ab. Desweiteren kann durch Einlassen einer gasförmigen $\left(\mathrm{CH}_{3}\right)_{3} \mathrm{Pt}\left(\mathrm{C}_{P} \mathrm{CH}_{3}\right)$ Verbindung mit Hilfe des Elektronen- oder Ionenstrahls eine chemische Reaktion hervorgerufen werden, so dass eine lokale Deposition von Platin an einer vorher bestimmten Position auf der Probe stattfindet. Die Pt- Deposition wird zum einen als Schutzfunktion genutzt, um die Probenoberfläche vor Ionenkontamination zu schützen, und desweiteren können mit Hilfe der Pt-Deposition präparierte Probenquerschnitte an Mikromanipulatoren befestigt werden und somit innerhalb der Kammer transferiert werden.

\subsection{Größenausschluss-Chromatographie (SEC)}

Zur Bestimmung von Molekulargewichten und Molmassenverteilungen von Polymeren ist die Gel-Permeations-Chromatographie (GPC) oder GrößenausschlussChromatographie (engl. size exclusion chromatography, SEC) die am häufigsten angewandte Methode. In Zusammenarbeit mit dem Institut für physikalische Chemie der Universität Göttingen wurden systematisch die in dieser Arbeit untersuchten Polymere vermessen. Dazu werden zunächst die laserdeponierten Polymerschichten in Tetrahydrofuran (THF) gelöst, und anschließend durch ein poröses Trenngel geleitet. Dabei benötigen kleinere Moleküle eine längere Zeit für das Durchlaufen der Säulen, da ihnen ein größeres Volumen zur Verfügung steht als größeren Molekülen. Hierbei werden unterschiedliche Ankunftszeiten detektiert, welche anschließend mit Standards auf die Molekulargewichte kalibiert werden.

In dieser Arbeit wurde ein WATERS-System mit drei PSS-SDV-Säulen mit einer Parikelgröße von $5 \mu \mathrm{m}$ und Porendurchmessern von $10^{4} \mathrm{~nm}, 10^{2} \mathrm{~nm}$ und $10 \mathrm{~nm}$ verwendet. Hierbei wurde ebenfalls THF als Eluent mit einer Durchflussmenge von $1 \mathrm{~mL} / \mathrm{min}$ verwendet. Desweiteren wurde mit vier verschiedenen Eichstandards (POLYMER STANDARD SERVICE, PSS, $800 \mathrm{~g} / \mathrm{mol}<M_{P}<1,8 \quad 10^{6} \mathrm{~g} / \mathrm{mol}$ ) kalibriert. 


\subsection{Röntgennahkantenabsorptionsspektroskopie (NEXAFS)}

Die Röntgennahkantenabsorptionsspektroskopie (Near-Edge X-ray Absorption Fine Structure, NEXAFS) bietet die Möglichkeit die verwendeten Polymere hinsichtlich ihrer Bindungsstruktur zu untersuchen. Hierbei wurden in Zusammenarbeit mit dem LaserLaboratorium Göttingen e.V. systematisch die in dieser Arbeit verwendeten Polymere untersucht. Dafür wurden die Polymerschichten auf $50 \mathrm{~nm}$ dicken SiliziumnitridMembranen deponiert mit Schichtdicken zwischen $250 \mathrm{~nm}$ und $500 \mathrm{~nm}$. Dadurch war es möglich die Proben in Transmission zu untersuchen. Die Messapparatur wird in der Arbeit von Peter Grossmann [45] genau beschrieben.

\subsection{Profilometrie}

Zur Bestimmung der Schichtdicke einer laserdeponierten Probe wurde ein Profilometer verwendet. Hierbei wurde die Probe vor der Beschichtung an einer Stelle mit einem Kapton-Klebestreifen abgedeckt, so dass nach der Beschichtung durch das Entfernen des Klebestreifens eine Kante erzeugt wird. Mit Hilfe einer Verfahreinheit wird die Probe unter einer diamantbesetzten Spitze bewegt, und ein Höhenprofil der Probe wird erstellt. Die Spitze ist dabei mechanisch mit einer Spule eines Lineartransformators (LVDT) gekoppelt, wodurch die strominduzierten Signale der Spitzenbewegung in Höheninformationen umgewandelt werden. Desweiteren lassen sich Scangeschwindigkeit und -länge einstellen und je nach Materialklasse wird die Auflagekraft der Spitze zwischen $50 \mu \mathrm{N}$ und $150 \mu \mathrm{N}$ variiert. Durch die Bestimmung der Schichtdicke lässt sich die Depositionsrate der verwendeten Materialklasse bestimmen.

In dieser Arbeit wurde ein Profilometer der Firma Veeco vom Typ Dektak 150 verwendet. 


\subsection{Indenter}

Um die mechanischen Eigenschaften der Proben zu untersuchen wurde ein Indenter der Firma Fischer vom Typ HV 100 C XYm verwendet. Desweiteren wurde in Zusammenarbeit mit Inga Knorr ein Nanoindenter der Firma Agilent vom Typ G200 verwendet. Bei der Indentierung wird ein Prüfkörper bekannter Geometrie in die zu untersuchende Probenoberfläche gedrückt und die Kraft- und Wegdaten werden aufgezeichnet. Aus diesen Daten lassen sich mechanische Größen wie die Härte und der Elastizitätmodul der Proben bestimmen. 


\section{Eigenschaften verwendeter Polymere}

In diesem Kapitel werden die Eigenschaften der in dieser Arbeit verwendeten Polymere vorgestellt. In der Theorie hat sich gezeigt, dass der Elastizitätsmodul der Polymerkomponente Einfluss auf die Rauigkeit des Polymer/Metall-Schichtsystems hat. Daher wurden laserdeponierte Polymerschichten mit verschiedenen Elastitzitätsmoduln untersucht. Die Polymethylmethacrylat-Schichten (PMMA) sind dabei von besonderem Interesse aufgrund des geringen Elastizitätsmoduls, wie im Folgendem gezeigt wird. Desweiteren werden Polycarbonat- (PC) und Bisphenol-A-Dimetharcrylat-Schichten (BisDMA) untersucht, welche im Vergleich zu den PMMA-Schichten höhere Elastizitätsmoduln auszeichnen.

\subsection{Eigenschaften von Polymethylmethacrylat (PMMA)}

Polymethylmethacrylat gehört zu der Gruppe der Thermoplaste. Sein Monomer besitzt die Summenformel $\mathrm{C}_{5} \mathrm{H}_{8} \mathrm{O}_{2}$. Die Strukturformel einer Monomereinheit, welche in Abbildung 4.1 dargestellt ist, zeigt neben der Kohlenstoff-Hauptkette eine MethylesterSeitengruppe mit einer $\mathrm{C}=\mathrm{O}-$ Carbonyleinheit.

Bei der Herstellung einer PMMA-Schicht koppelt die Energie der Laserstrahlung (ca. $5 \mathrm{eV}$ ) einer Wellenlänge von $248 \mathrm{~nm}$ innerhalb des bestrahlten Targets lediglich mit der Doppelbindung des PMMA-Monomers. Die Laserstrahlung dringt dabei ungefähr $150 \mu \mathrm{m}$ tief ins Material ein [46]. Die geringe Anzahl an Doppelbindungen sorgt dafür, dass nur ein geringer Teil der UV-Strahlung im Polymertarget absorbiert wird, und der Abtrag zunächst sehr gering ist. Somit müssen erst photochemische Prozesse im Target ablaufen, um eine ausreichend hohe Depositionsrate zu erzielen [47,48]. Durch die 


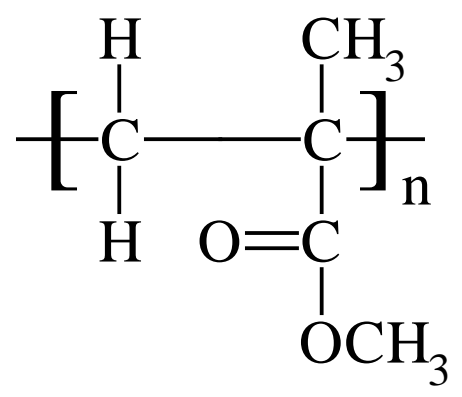

Abbildung 4.1: Strukturformel von PMMA. Die Absorption der UV-Strahlung ist vor der Inkubation des Targets aufgrund lediglich einer Doppelbindung (Carbonyl-Einheit, $\mathrm{C}=\mathrm{O}$ ) sehr gering.

einfallende UV-Strahlung kann die absorbierte Energie benachbarte Einfachbindungen brechen, was, wie in der Strukturformel zu erkennen ist, zum Abreißen von Teilen der Seitengruppe führen kann. Desweiteren kann es zur Spaltung der Hauptkette kommen, wodurch weitere Absorptionszentren für die UV-Strahlung in Form von $\mathrm{C}=\mathrm{C}$ Doppelbindungen entstehen. Dieser Prozess ist in der Literatur mit dem Begriff der Inkubation bereits bekannt, und wurde von Lippert et al. grundlegend untersucht [49-51].
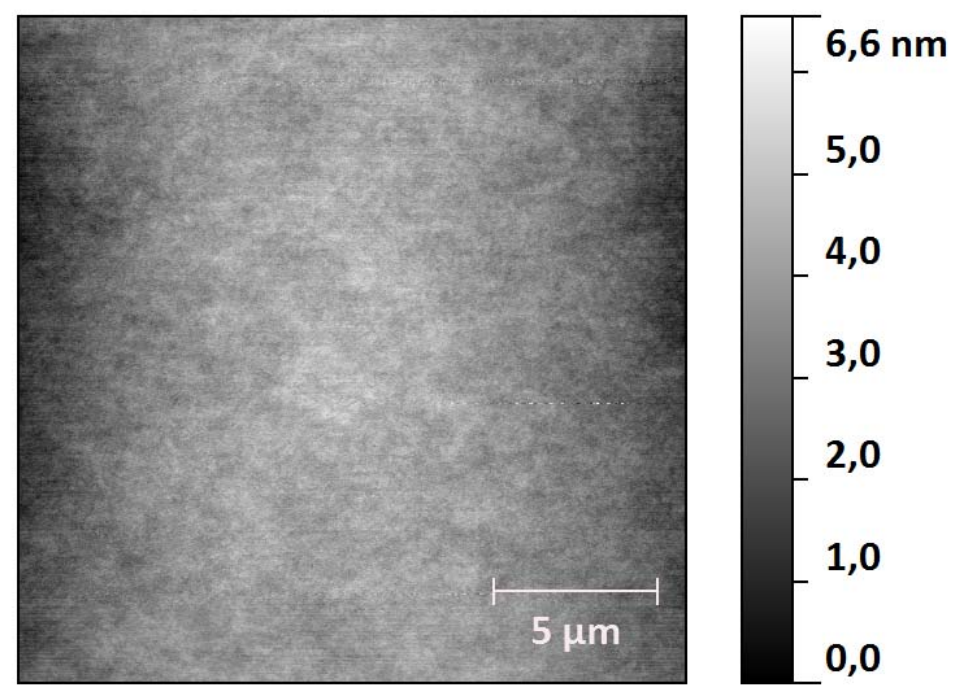

Abbildung 4.2: AFM-Messung einer glatten 5,5 $\mu \mathrm{m}$ dicken PMMA-Schicht mit einer RMS-Rauigkeit von $0,3 \mathrm{~nm}$.

Stellt man nun mittels gepulster Laserdeposition bei einer niedrigen Energiedichte von $77 \mathrm{~mJ} / \mathrm{cm}^{2}$ eine 5,5 $\mathrm{m}$ dicke PMMA-Schicht her, so zeigt diese im AFM eine äußerst glatte Oberfläche mit einer Rauigkeit von nur 0,3 nm (siehe Abbildung 4.2). Um diese Rauigkeit besser einordnen zu können, sei hier erwähnt, dass herkömmliche 
Si-Substrate dieselbe Rauigkeit besitzen. Über eine Fläche von $20 \mu^{2}$ sind keine Droplets oder anderweitige Rauigkeiten zu erkennen, was den glatten Charakter dieser Schichten gut wiedergibt. Dies ist nur möglich durch die Verwendung einer niedrigen Laserenergiedichte, welche sich nahe an der Depositionsschwelle $\left(56 \mathrm{~mJ} / \mathrm{cm}^{2}\right)$ befindet. Bei der Verwendung einer höheren Laserenergiedichte steigt die Dropletdichte stark an, so dass man eine zweikomponentige Schicht vorliegen hat [52]. Die niedrige Laserenergiedichte konnte durch die Aufweitung des Brennflecks erzeugt werden, und die Depositionsrate betrug unter diesen Bedingungen 0,2 nm/Puls.

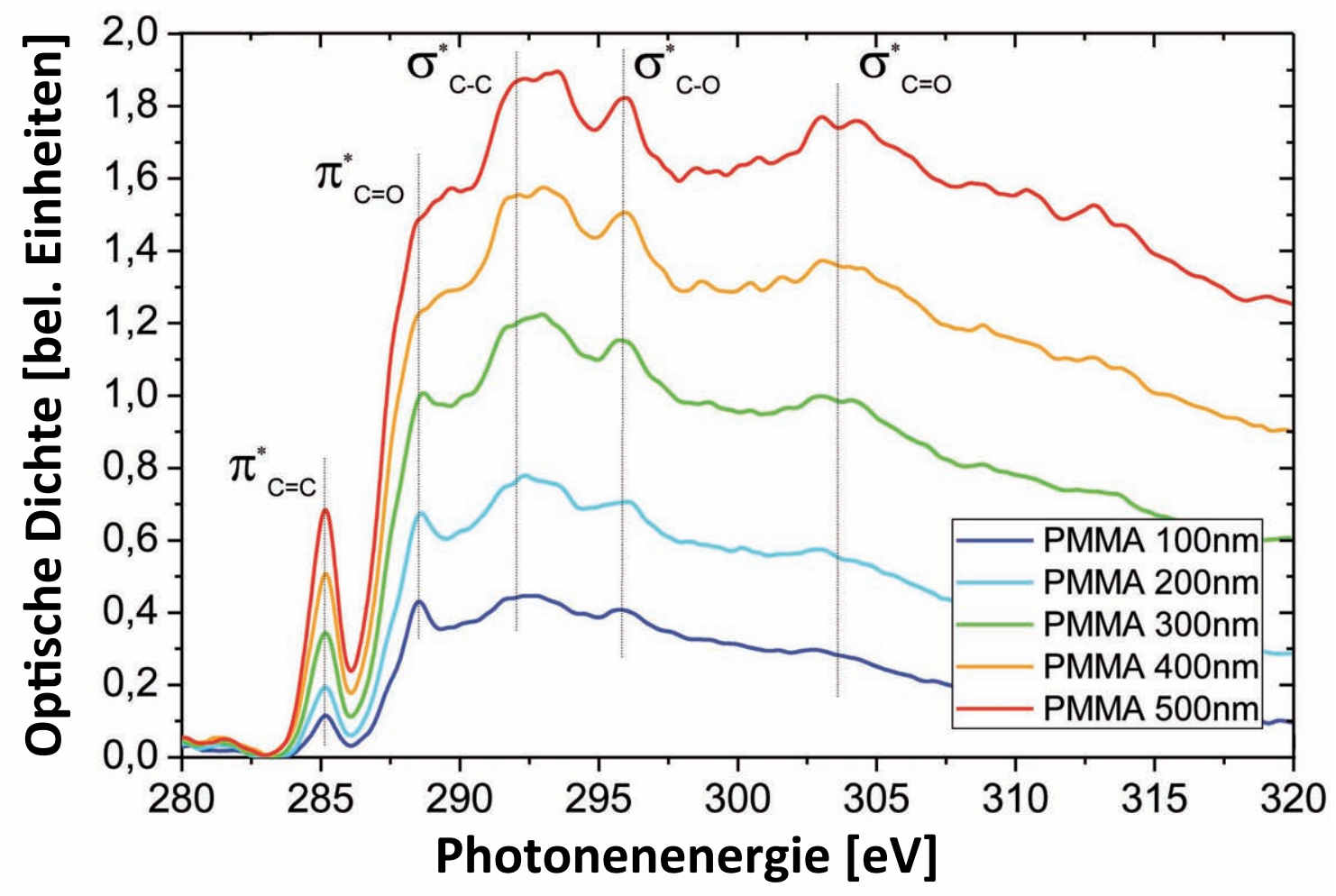

Abbildung 4.3: NEXAFS-Messungen von fünf PMMA-Schichten unterschiedlicher Schichtdicken $(100 \mathrm{~nm}, 200 \mathrm{~nm}, 300 \mathrm{~nm}, 400 \mathrm{~nm}$ und $500 \mathrm{~nm})$. Es zeigt sich, dass mit zunehmender Schichtdicke die Intensität für die $\mathrm{C}=\mathrm{C}$-Doppelbindungen zunimmt (Zusammenarbeit mit Peter Großmann [45]).

Um die chemischen Veränderungen am PMMA-Target während der Inkubation genauer zu untersuchen, wurden PMMA-Schichten unterschiedlicher Schichtdicken von $100 \mathrm{~nm}$ bis $500 \mathrm{~nm}$ nacheinander hergestellt. Dabei wurde mit der $100 \mathrm{~nm}$ dicken Schicht begonnen und die Schichtdicke wurde im folgenden kontinuierlich erhöht. Die hergestellten PMMA-Schichten wurden anschließend in Zusammenarbeit mit Peter Großmann im Laser-Laboratorium Göttingen mit RöntgennahkantenabsorptionsspektroskopieMessungen (Near-Edge X-ray Absorption Fine Structure, NEXAFS) untersucht. Die 
NEXAFS-Messungen zeigen, dass schon bei der $100 \mathrm{~nm}$ dicken PMMA-Schicht $\mathrm{C}=\mathrm{C}$ Doppelbindungen vorhanden sind und die $\mathrm{C}=\mathrm{O}$-Doppelbindungen bei einer Photonenenergie von $288 \mathrm{eV}$ noch existieren. Mit zunehmender Schichtdicke nimmt die Intensität für die $\mathrm{C}=\mathrm{C}$-Doppelbindungen $\mathrm{zu}$, die Intensität der $\mathrm{C}=\mathrm{O}$-Doppelbindung wird jedoch immer kleiner. Desweiteren wachsen mit zunehmender Schichtdicke auch die Intensitäten der C-C-Einfachbindung und der C-O-Einfachbindung. Die starke Abnahme der Intensität der $\mathrm{C}=\mathrm{O}$-Doppelbindungen bestätigt, dass es während der Inkubation verstärkt zum Abspalten von Seitengruppen und einer Zunahme von Absorptionszentren in Form von $\mathrm{C}=\mathrm{C}$-Doppelbindungen kommt. Insgesamt kann der Inkubationsprozess anhand der NEXAFS-Messungen also gut nachvollzogen werden. Zusätzlich haben die Messungen gezeigt, dass erst nach einer Anzahl von 2000 Laserpulsen der Inkubationsprozess abgeschlossen ist, da sich hier die Spektren nicht mehr allzu stark voneinander unterscheiden. Um reproduzierbare Ergebnisse zu erhalten, wurde das Target daher bei allen späteren Experimenten vor einer Schichtherstellung immer zunächst mit 2000 Laserpulsen eingeschossen.

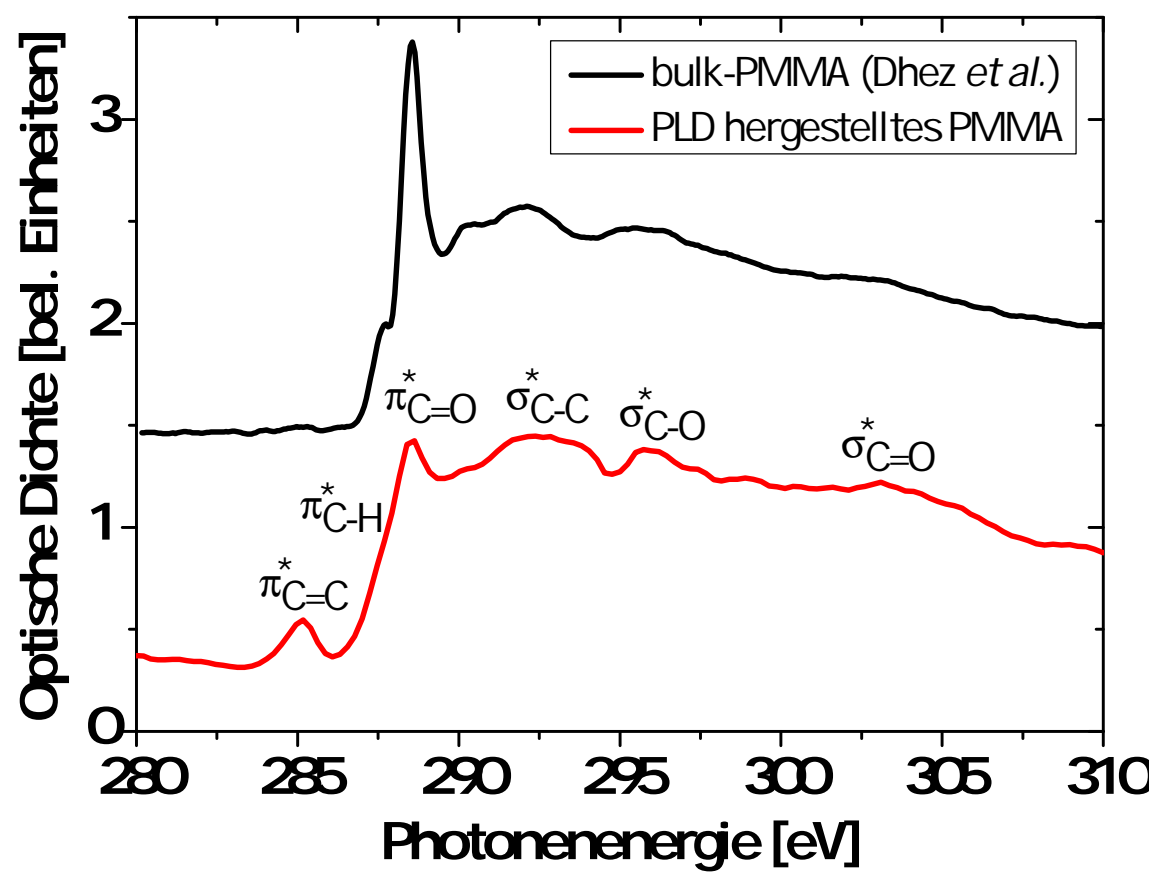

Abbildung 4.4: NEXAFS-Messungen einer $300 \mathrm{~nm}$ dicken laserdeponierten PMMA-Schicht im Vergleich mit bulk-PMMA [53]. Die Schicht weist alle typischen Resonanzen auf, welche auch das bulk-Material zeigt. Allerdings zeigt sich neben der Existenz von $\mathrm{C}=\mathrm{C}$-Doppelbindungen auch eine Verringerung der Intensität der Carbonyl-Einheit $(\mathrm{C}=\mathrm{O})$, was auf ablationsbedingte Modifikationen des PMMAs während der Inkubation schließen lässt (Daten aus [54]). 
Vergleicht man nun NEXAFS-Messungen aus der Literatur von bulk-PMMA (Daten aus [53]) mit einer $300 \mathrm{~nm}$ dicken laserdeponierten PMMA-Schicht, zeigt sich, dass bei der PMMA-Schicht alle typischen Resonanzen vorhanden sind (siehe Abbildung 4.4). Im Gegensatz zum bulk-Material weist das Spektrum der PMMA-Schicht den Peak der $\mathrm{C}=\mathrm{C}$-Doppelbindung auf, was den Inkubationsprozess und die resultierende Neubildung der $\mathrm{C}=\mathrm{C}$-Doppelbindungen bestätigt. Desweiteren lässt sich beim direkten Vergleich die bereits diskutierte Abschwächung der $\mathrm{C}=\mathrm{O}-$ Resonanz bei der PMMASchicht erkennen, welche durch das Abreißen von Teilen der Seitengruppe erklärt werden kann.

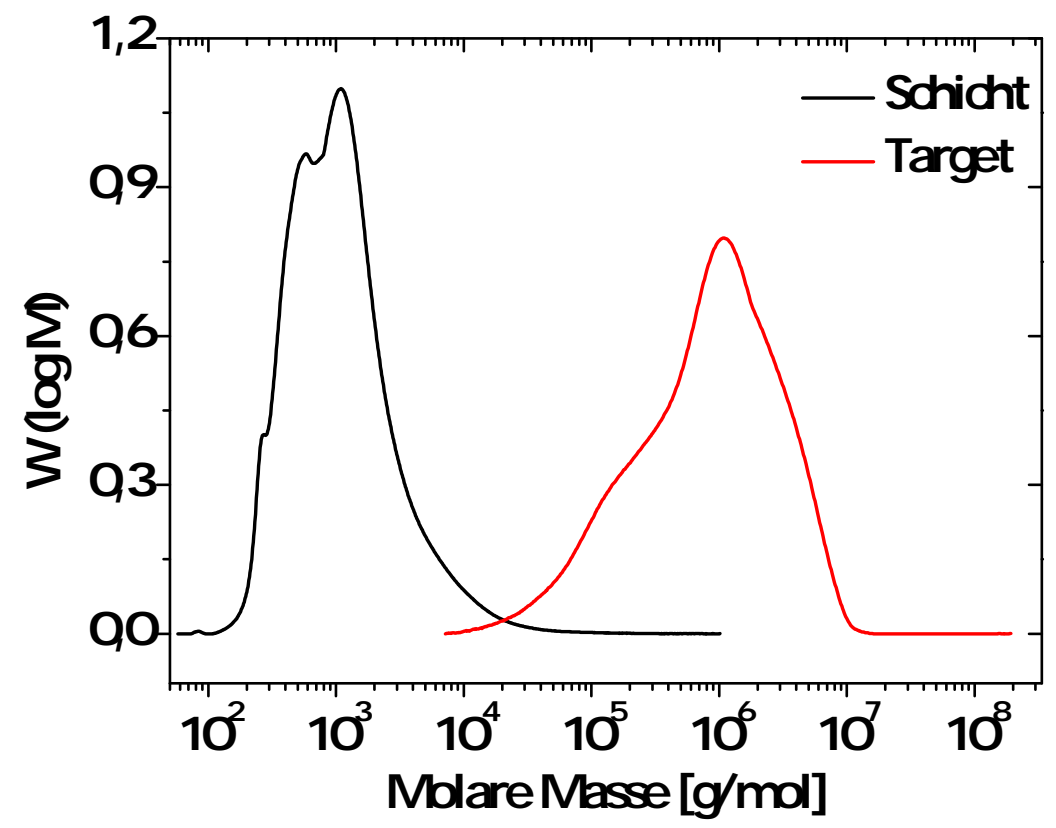

Abbildung 4.5: SEC-Messungen eines PMMA-Targets und einer PMMA-Schicht. Die mittlere Molmasse beträgt dabei für das PMMA-Target $M_{w}=1,4010^{6} \mathrm{~g} / \mathrm{mol}$ und für die PMMASchicht $M_{w}=1,9010^{3} \mathrm{~g} / \mathrm{mol}$ und ist somit um drei Größenordnungen kleiner als die des Ausgangsmaterials, welches sich durch den Ablationsprozess erklären lässt (Zusammenarbeit mit Robert Rotzoll).

Als Folge der Bildung der $\mathrm{C}=\mathrm{C}$-Doppelbindungen wird die Laserstrahlung effektiv in diesen Bindungen absorbiert, was zur Brechung dieser oder benachbarter Bindungen und damit zur Verkürzung der langen Polymerketten im Target führen kann. Nach der Ablation besteht daher die deponierte PMMA-Schicht selbst aus sehr kurzen Polymerketten. In Zusammenarbeit mit der Chemie (Robert Rotzoll) konnten die Kettenlängen sowohl vom Targetmaterial als auch von der hergestellten Schicht mit Hilfe der Größenausschluss-Chromatographie (size exclusion chromatography, SEC) 
bestimmt werden (siehe Abbildung 4.5). Als Targetmaterial wurde eine PMMA-Platte der Firma Goodfellow verwendet. Die SEC-Messungen zeigen, dass das Targetmaterial eine mittlere Molmasse von $M_{w}=1,4010^{6} \mathrm{~g} / \mathrm{mol}$ beträgt, wohingegen die hergestellte Schicht eine mittlere Molmasse von lediglich $M_{w}=1,9010^{3} \mathrm{~g} / \mathrm{mol}$ aufweist und somit um drei Größenordnungen kleiner ist. Die SEC-Messungen bestätigen somit, dass die Ketten während des Ablationsprozesses um einen Faktor von etwa 1000 verkürzt werden. Insgesamt ist das PMMA zwar modifiziert, weist jedoch noch für das PMMA charakteristische Eigenschaften auf, was neben obiger NEXAFS-Messungen auch anhand von Fouriertransformations-Infrarotspektroskopie (FT-IR) gezeigt werden konnte [55].

Die verkürzten Ketten haben auch eine Absenkung der Glasübergangstemperatur zur Folge, wie von O'Driscoll und Sanayei sowohl für PMMA als auch für weitere Polymere untersucht worden ist [56]. Diese Absenkung der Glasübergangstemperatur hat weiterhin auch Auswirkung auf die Viskosität der PMMA-Schichten. So kann mit Hilfe der Mark-Houwink-Gleichung die Viskosität $\eta$ in Beziehung mit der bestimmten mittleren Molmasse $M_{w}$ gebracht werden $[57,58]$.

$$
\eta=K M_{w}^{a}
$$

Hierbei bezeichnet $K$ eine Konstante und für $a$ wird ein Wert in der Literatur von $a=3$, 4 für PMMA angegeben [59]. Setzt man nun die mittlere Molmasse der Schicht ins Verhältnis zur mittleren Molmasse des Targetmaterials, so ergibt sich für die Viskosität beider Materialien folgendes Verhältnis:

$$
\frac{\eta_{\text {Schicht }}}{\eta_{\text {Target }}}=\frac{M_{w_{\text {Schicht }}}^{3,4}}{M_{w_{\text {Target }}}^{3,4}} \quad 1,8 \quad 1010
$$

In der folgenden Tabelle 4.1 sind beispielhaft Werte für die Viskosität von verschiedenen Materialien dargestellt. Dadurch lässt sich für das PMMA-Targetmaterial ein Wert für die Viskosität von $\eta_{\text {Target }} \quad 10^{12}$ Pas abschätzen. Über die obige Relation würde sich daher für die laserdeponierte Schicht folgende Viskosität ergeben:

$$
\eta_{\text {Schicht }} \quad 1,8 \quad 10^{10} \quad 10^{12} \mathrm{Pas} \quad 180 \mathrm{Pas}
$$

Dieser Abschätzung zurfolge sollte demnach die Viskosität der Schicht aufgrund der Kettenverkürzung um zehn Größenordnungen geringer sein als die des Ausgangsmateri- 
als. Eine Viskosität von lediglich 180 Pas ist dabei vergleichbar mit einem dickflüssigem Sirup [57] (siehe Tabelle 4.1).

\begin{tabular}{|l|c|}
\hline Material & Viskosität $\eta$ [Pas] \\
\hline Luft & $10^{5}$ \\
Wasser & $10^{3}$ \\
Polymer Latex & $10^{2}$ \\
Olivenöl & $10^{1}$ \\
Glyzerin & $10^{0}$ \\
Sirup & $10^{2}$ \\
Polymer Schmelzen & $10^{2} 10^{6}$ \\
Pech & $10^{9}$ \\
Plastik & $10^{12}$ \\
Glas & $10^{21}$ \\
\hline
\end{tabular}

Tabelle 4.1: Viskositäten verschiedener Materialien (Daten aus [57])

Desweiteren werden auch die mechanischen Eigenschaften der Schicht durch die verkürzten Ketten verändert, wie im Folgenden gezeigt wird. In Abbildung 4.6 sind die bei Indentierung gemessenen Kraft-Weg-Kurven einer PMMA-Schicht und des PMMA-Targets im Vergleich gezeigt. Zunächst wurden auf die Proben innerhalb von 20 s eine Kraft von $300 \mathrm{mN}$ aufgebracht. Anschließend wurde die Kraft für 5 s konstant gehalten und danach innerhalb von 20 s entladen. Die Kraft-Weg-Kurve für das PMMATarget zeigt am Anfang einen sehr kleinen elastischen Bereich und danach plastische Verformung. Aus der Entladungskurve konnte ein Wert für den E-Modul von $4 \mathrm{GPa}$ angegeben werden. Die Kraft-Weg-Kurve für die PMMA-Schicht zeigt schon bei einer geringen Krafteinwirkung ein tiefes Eindringen des Indenters an. Desweiteren weist die Entladungskurve eine negative Steigung auf, was die Bestimmung des E-Moduls somit nicht möglich macht. Daher wurde in Zusammenarbeit mit Timo Wuttke das Kriechverhalten der PMMA-Schichten untersucht. Es konnte gezeigt werden, dass ein viskoelastisches Verhalten vorliegt [60]. Jedoch war es nicht möglich mit Hilfe von Nanoindentermessungen den genauen E-Modul-Wert der PMMA-Schichten zu bestimmen. Es konnte lediglich eine obere Abschätzung von $300 \mathrm{MPa}$ vorgenommen werden, welches die untere Messgrenze des Nanoindenters darstellt.

Eine weitere Methode, die es möglich macht insbesondere weiche Polymerschichten zu vermessen, ist die Verwendung eines AFMs als Indenter. So zeigen z.B. Domke und Radmacher, dass mit einem AFM als Indenter sogar dünne Schichten aus Gelatine mit einem E-Modul von $20 \mathrm{kPa}$ vermessen werden können [61]. 


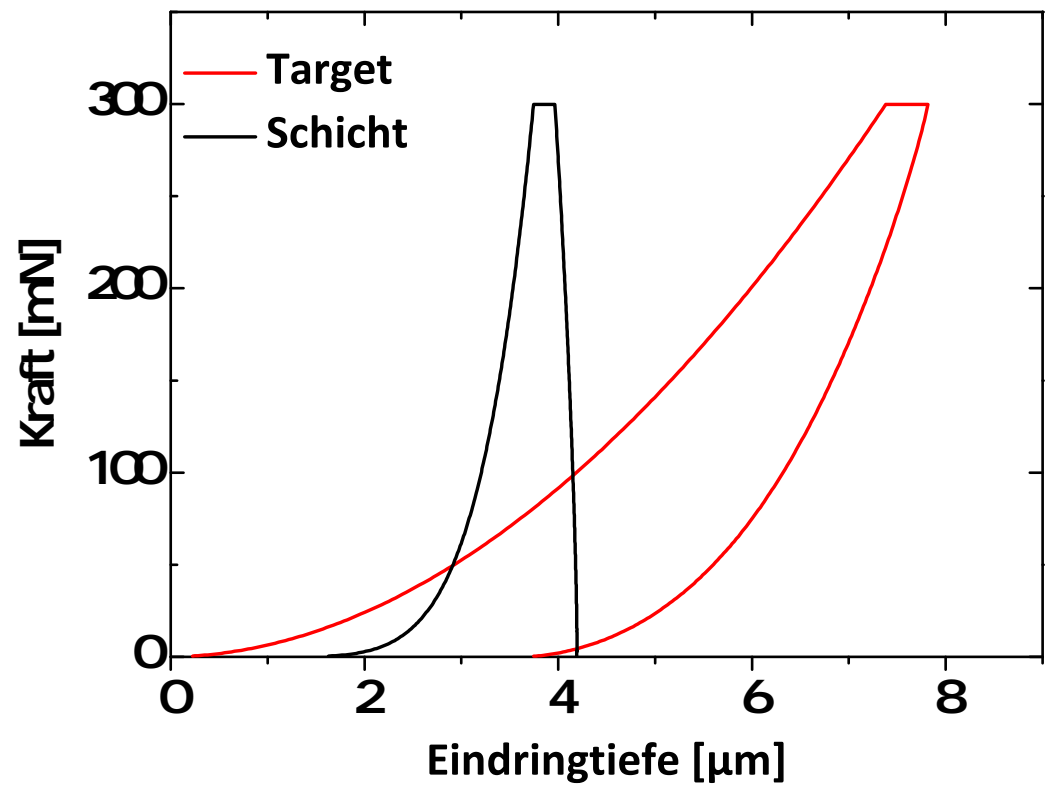

Abbildung 4.6: Kraft-Weg-Kurven für eine PMMA-Schicht und ein PMMA-Target. Die Kurve für das Targetmaterial zeigt den typischen Verlauf und man erhält einen E-Modul-Wert von 4 GPa. Für die Schicht zeigt die Entladungskurve eine negative Steigung, was die Bestimmung des E-Moduls nicht möglich macht.

In Zusammenarbeit mit Matthias Büchsenschütz-Göbeler wurden AFMIndentermessungen an $10 \mu \mathrm{m}$ dicken PMMA-Schichten auf Silizium-Substraten zur Bestimmung des E-Moduls durchgeführt. Hierbei wurde jeweils eine Kraft von $260 \mathrm{nN}$ auf die Schichten aufgebracht. In Abbildung 4.7 sind die Beladungskurven gezeigt, bei denen die PMMA-Schichten wegen des viskoelastischen Verhaltens mit verschiedenen Verformungsgeschwindigkeiten vermessen wurden. Betrachtet man die Messung der PMMA-Schicht mit der höchsten Verformungsgeschwindigkeit von $7810 \mathrm{~nm} / \mathrm{s}$, so zeigt die Beladungskurve zunächst einen linearen Anstieg, welcher das elastische Verhalten der PMMA-Schicht wiedergibt. Das viskoelastische Verhalten der PMMA-Schicht hat zur Folge, dass der Elastizitätsmodul stark frequenzabhängig ist. Somit konnte ein Wert für den E-Modul nur abgeschätzt werden. Anhand von mehreren Messungen bei diesen hohen Verformungsgeschwindigkeiten ergaben sich Werte in einem Bereich von $150 \mathrm{MPa}$. Bei niedrigeren Verformungsgeschwindigkeiten ist ein Abknicken der Beladungskurven zu beobachten und plastische Verformung findet statt. Je langsamer die Probe verformt wird, desto eher beginnt die Beladungskurve abzuknicken. 


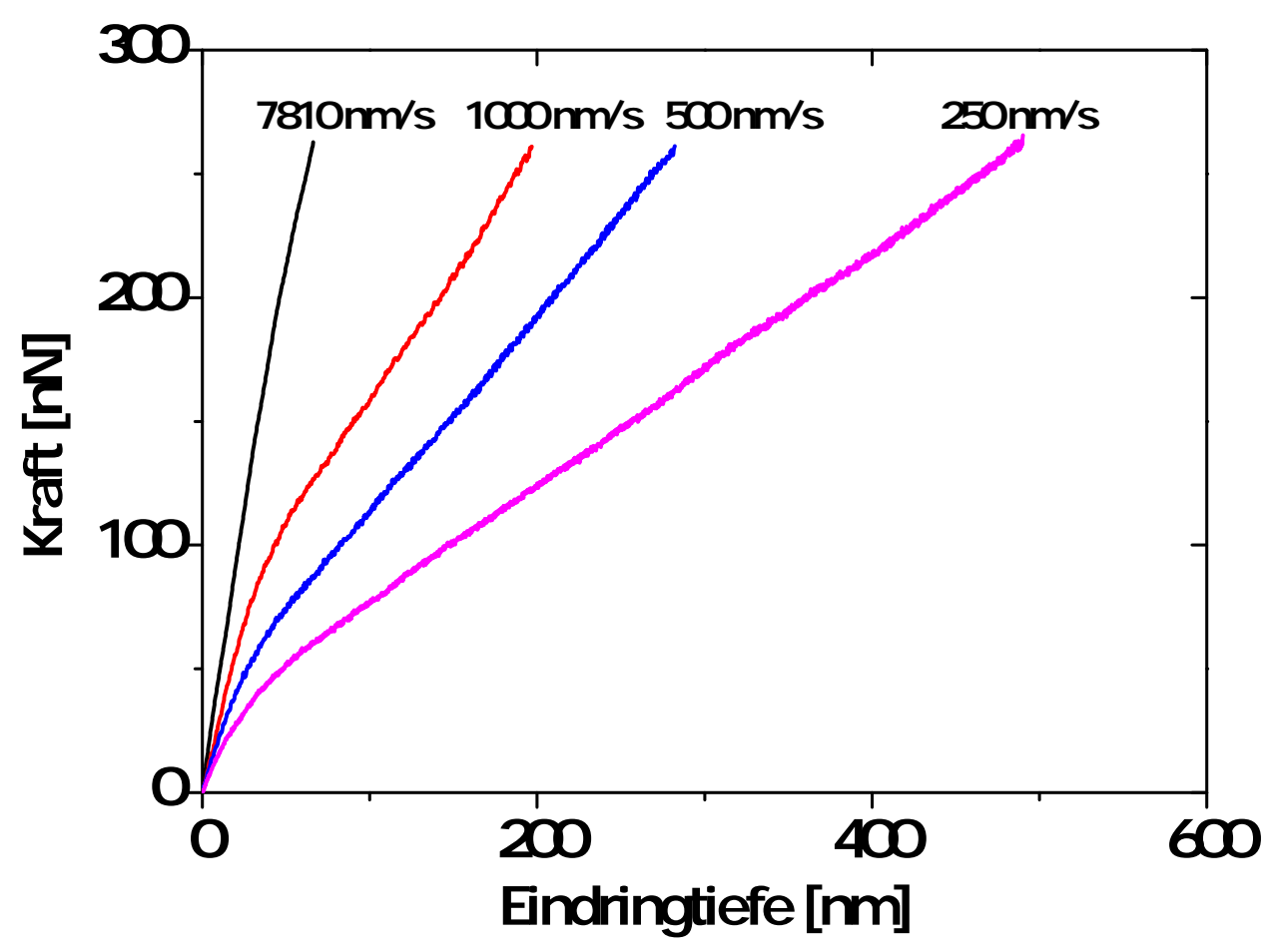

Abbildung 4.7: AFM-Indentermessungen an einer $10 \mu \mathrm{m}$ dicken PMMA-Schicht auf einem Si-Substrat. Verschiedene Verformungsgeschwindigkeiten zeigen, dass das Verhalten der PMMA-Schicht für schnelle Verformungsgeschwindigkeiten einen eher elastischen Charakter aufweist (Zusammenarbeit mit Matthias Büchsenschütz-Göbeler).

Um das Kriechverhalten der PMMA-Schichten auf langen Zeitskalen zu untersuchen, wurden Relaxationsmessungen durchgeführt. Hierbei wurde eine konstante Kraft von $735 \mathrm{nN}$ auf die PMMA-Probe ausgeübt und die Zeit gemessen, in der die Probe relaxiert. In Abbildung 4.8 ist sowohl die Messung an einer PMMA-Schicht als auch vergleichsweise an einer Poly(dimethylsiloxan)-Schicht (PDMS) gezeigt. Die PMMASchicht zeigt nach Aufbringen der Kraft einen schnellen Abfall der Kurve. Innerhalb weniger Sekunden fällt die Kraft aufgrund von Fließprozessen auf nahezu Null zurück. Schließlich übt die Probe keinen Widerstand mehr gegen den Cantilever aus. Die PMMA-Schicht verhält sich also auf langen Zeitskalen wie eine viskose Flüssigkeit. Im Gegensatz dazu zeigt die PDMS-Probe nach Aufbringen einer Kraft von $280 \mathrm{nN}$ auf einer ähnlichen Zeitskala von wenigen Sekunden nur einen minimalen Abfall der Kurve.

Zusammenfassend konnte in diesem Kapitel gezeigt werden, dass das PMMATarget ein schwacher Absorber ist, und dass die laserdeponierten PMMA-Schichten um drei Größenordnungen verkürzte Kettenlängen besitzen. Einer Abschätzung zur 

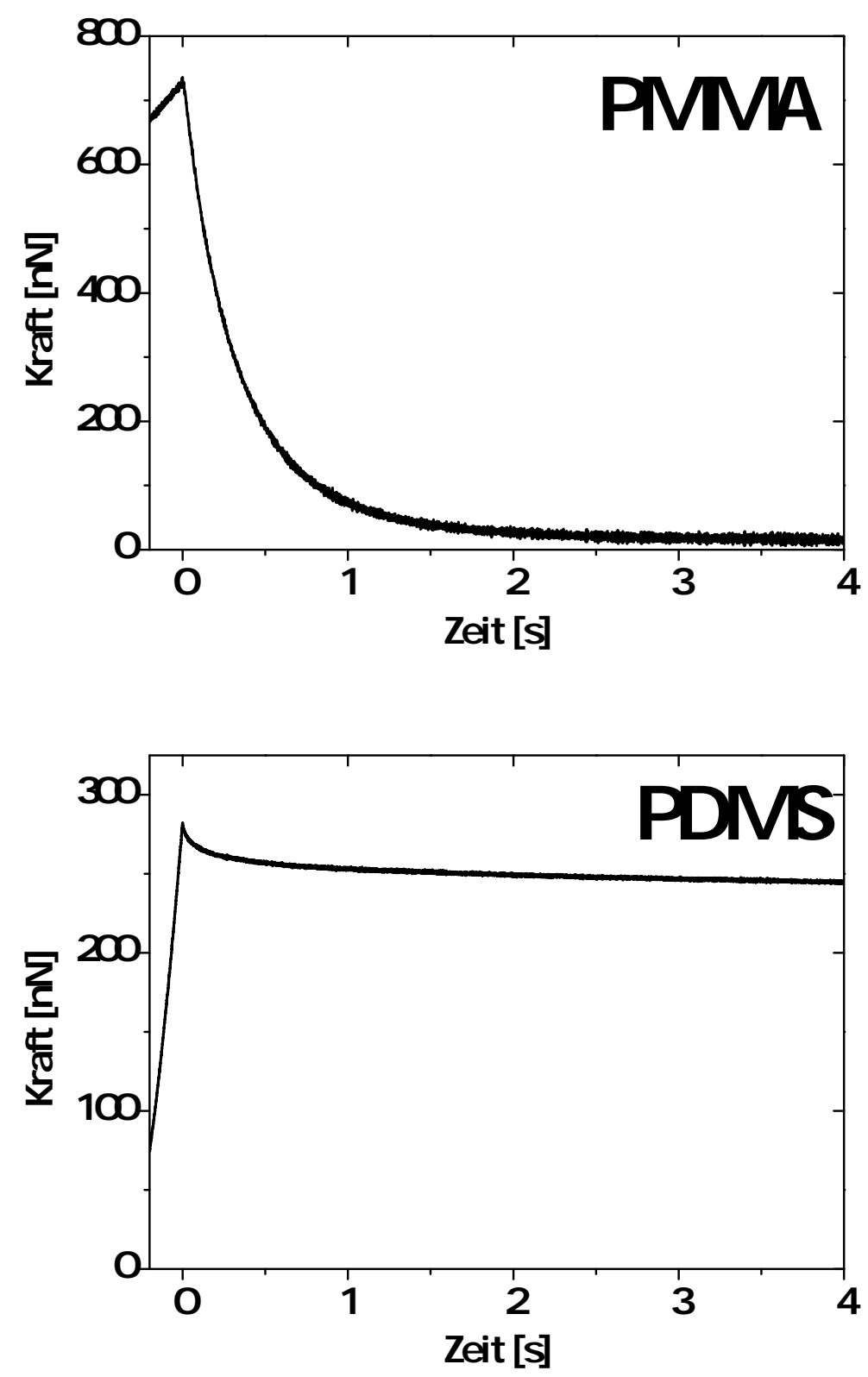

Abbildung 4.8: AFM-Indentermessungen einer $10 \mu \mathrm{m}$ dicken PMMA Schicht und zum Vergleich einer PDMS-Referenzprobe. Es zeigt sich, dass das PMMA auf langen Zeitskalen das Verhalten einer viskosen Flüssigkeit aufweist, da es der angelegten Kraft keinen Widerstand leistet. Im Vergleich zeigt das PDMS viskoelastisches Verhalten (Zusammenarbeit mit Matthias Büchsenschütz-Göbeler).

Folge ist demnach die Viskosität der Schichten um zehn Größenordnungen geringer als die des Ausgangsmaterials. Aus AFM-Indentermessungen wurde ein niedriger Elastizitätsmodul im unteren MPa-Bereich bestimmt. Desweiteren weisen die PMMASchichten ein viskoelastisches Verhalten auf, wobei sich die Schichten auf kurzen 
Zeitskalen wie ein elastischer Festkörper und auf langen Zeitskalen ( $>1$ s) wie eine viskose Flüssigkeit verhalten.

\subsection{Eigenschaften von Bisphenol-A-Dimethacrylat (BisDMA) und Polycarbonat (PC)}

In diesem Kapitel werden nun zwei weitere Polymere, Bisphenol-A-Dimethacrylat (BisDMA) und Polycarbonat (PC), vorgestellt. Aufgrund der Vielzahl an $\mathrm{C}=\mathrm{C}$ Doppelbindungen innerhalb der Phenolringe und diverser Carbonyl-Einheiten $(\mathrm{C}=\mathrm{O})$ zeichnen sich beide Polymere, deren chemische Strukturformel nach Abbildung 4.9 zudem sehr ähnlich sind, durch eine hohe Absorption der eingestrahlten Laserenergie aus.<smiles>C=C(C)C(=O)Oc1ccc(C(C)C(=O)Oc2ccc(C(C)(C)c3ccc(OC(=O)C(=C)C)cc3)cc2)cc1</smiles>

Abbildung 4.9: Strukturformel von BisDMA (links) und PC (rechts). Beide Polymere zeichnen sich durch eine Vielzahl von Doppelbindungen $(\mathrm{C}=\mathrm{O}$ und $\mathrm{C}=\mathrm{C})$ aus, was zu einem hohen Absorptionsgrad der UV-Strahlung führt.

Die NEXAFS-Spektogramme von acht nacheinander hergestellten PC-Schichten, wobei jeweils 250 Laserpulse auf das PC-Target abgegeben wurden, sind wie Abbildung 4.10 zeigt, allesamt nahezu identisch. Dies zeigt, dass in den Frühstadien des Ablationsprozesses beim PC keine Inkubation stattfindet. Dies bestätigen auch die von Anfang an stabilen Depositionsraten von 0,2 nm/Puls.

Weiterhin wird wie auch bei der Ablation von PMMA eine sehr niedrige Laserenergiedichte von $77 \mathrm{~mJ} / \mathrm{cm}^{2}$ gewählt, um glatte dropletfreie Schichten herzustellen. In Abbildung 4.11 sind zwei AFM-Messungen einer BisDMA-Schicht (links) und einer PC-Schicht (rechts) gezeigt. Beide Schichten sind glatt mit einer niedrigen RMSRauigkeit von 2,6 nm (BisDMA) und 2,9 nm (PC). Allerdings weisen beide Schichten im Vergleich zur PMMA-Schicht mehr Rauigkeiten aufgrund von Droplets auf. Für alle drei Polymere wurde stets die gleiche Laserenergiedichte verwendet, was der Grund 


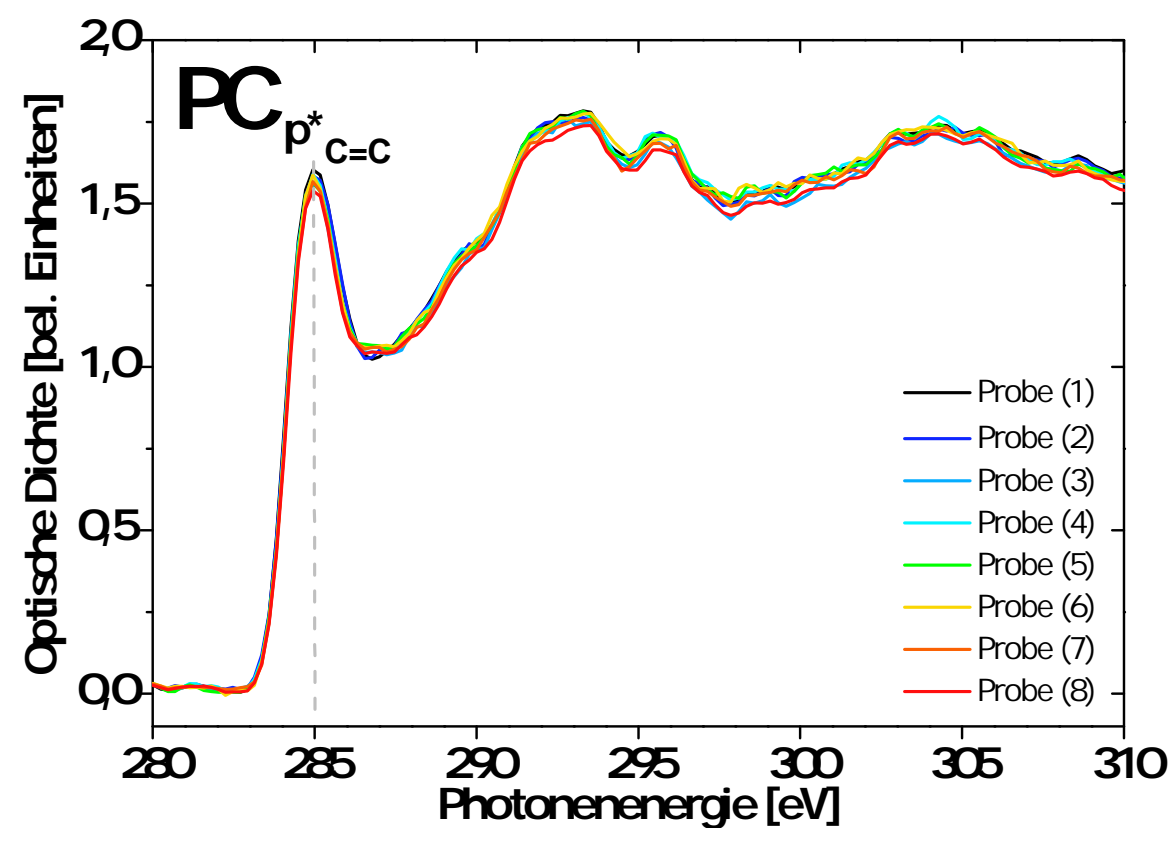

Abbildung 4.10: NEXAFS-Messungen mehrerer PC-Schichten, welche alle mit 250 Laserpulsen nacheinander hergestellt wurden. Die Ähnlichkeit aller Spektren zeigt, dass in den Frühstadien des Ablationsprozesses keine Inkubation stattfindet (Zusammenarbeit mit Peter Großmann).
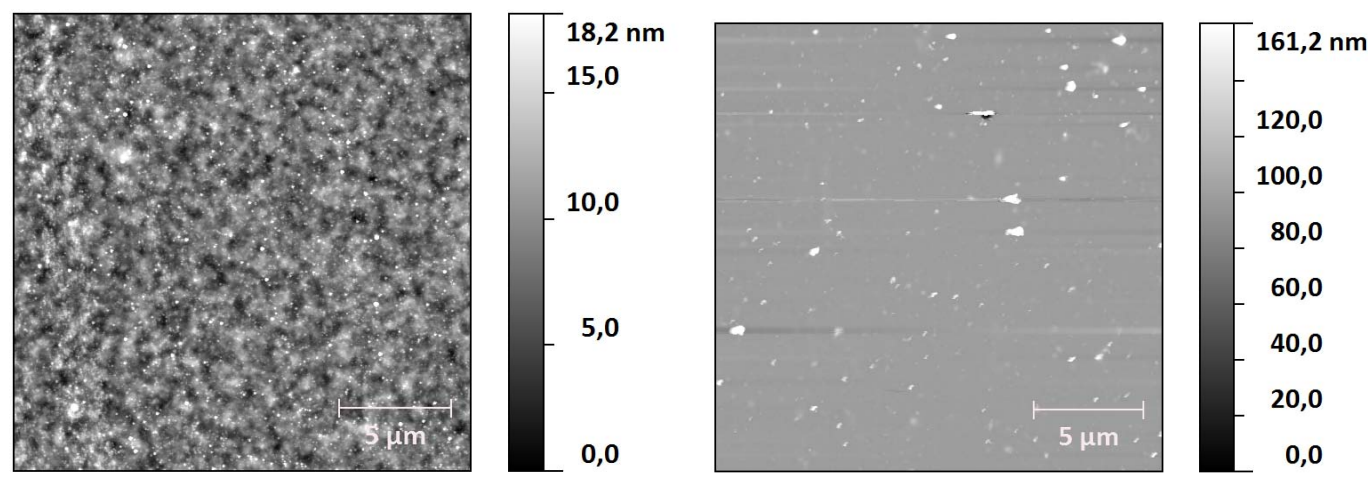

Abbildung 4.11: AFM-Messungen einer BisDMA-Schicht (links) und einer PC-Schicht (rechts). Beide Polymerschichten zeigen eine sehr geringe Oberflächenrauigkeit.

für die vermehrten Droplets sowohl auf den BisDMA- als auch auf den PC-Schichten sein kann, da diese Laserenergiedichte anscheinend nicht $\mathrm{zu}$ einer maximalen (aber ausreichenden) Reduzierung der Droplets führt.

Die BisDMA-Targets bestanden aus Pulver der Firma Sigma Aldrich, welche mit Hilfe einer Einbettpresse zu festen Targets gepresst wurden. Die mittlere Molmasse des Pulvers beträgt $M_{w}=3,710^{2} \mathrm{~g} / \mathrm{mol}$. Als Ausgangsmaterial für die PC-Schichten wurde eine PC-Platte der Firma Goodfellow verwendet. Für beide Polymere konnten 
keine Angaben über die Kettenlängen in den deponierten Schichten gemacht werden, da aufgrund der starken Vernetzung der Schichten, diese nicht vom Substrat gelöst werden konnten und somit keine SEC-Messungen möglich waren.
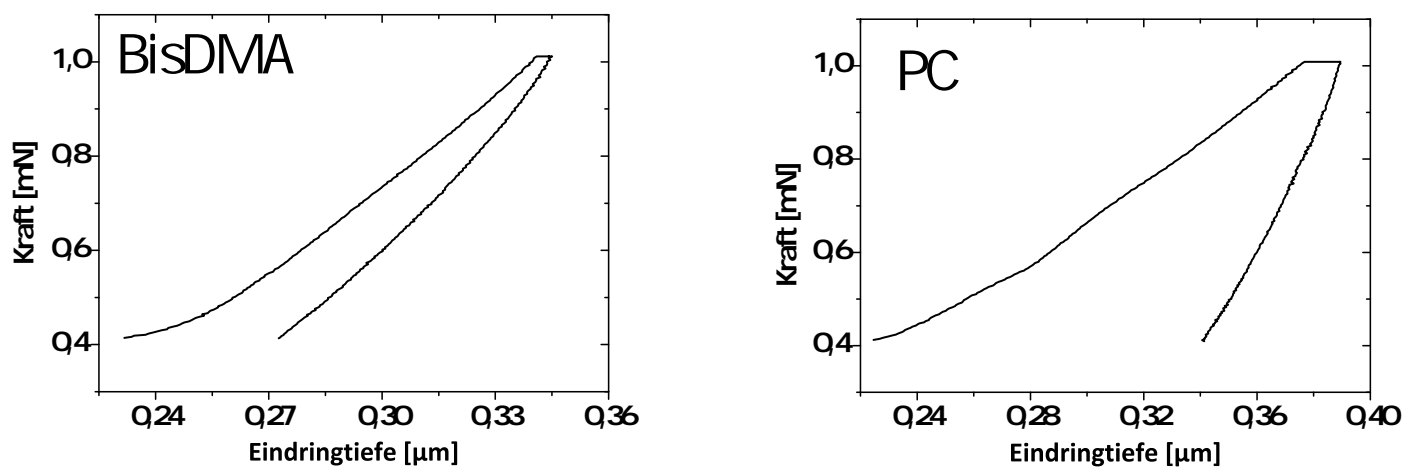

Abbildung 4.12: Kraft-Weg-Kurven für eine BisDMA-Schicht (links) und eine PC-Schicht (rechts). Aus beiden Kurvenverläufen lässt sich ein Wert für den Elastizitätsmodul von 6 GPa berechnen.

Der hohe Vernetzungsgrad der Schichten zeigt sich insbesondere in den mechanischen Eigenschaften der beiden Polymere. In Abbildung 4.12 sind Kraft-Weg-Kurven von Indentationsmessungen sowohl für die BisDMA-Schicht (links) als auch für die PCSchicht (rechts) gezeigt. Beide Schichten wurden mit einer Kraft von $1 \mathrm{mN}$ beladen. Im Vergleich zur PMMA-Schicht sind die Entladungskurven für die BisDMA- und die PCSchicht von positiver Steigung und machen es somit möglich den Elastizitätsmodul der Schichten zu messen. Für die BisDMA- und auch die PC-Schicht ergibt sich ein Wert für den Elastizitätsmodul von $6 \mathrm{GPa}$. Auch hier zeigt sich wieder, dass beide Polymere sich in ihren mechanischen Eigenschaften sehr ähnlich sind.

Zusammenfassend konnte gezeigt werden, dass bei den Polymeren BisDMA und PC schon im Targetmaterial viele $\mathrm{C}=\mathrm{C}$-Doppelbindungen vorliegen, wodurch eine gute Absorption der UV-Strahlung gegeben ist. Desweiteren besitzen beide Polymerschichten einen E-Modul von etwa $6 \mathrm{GPa}$, welcher im Vergleich zu den PMMA-Schichten deutlich größer ist. Aufgrund des aufwendigeren Herstellungsverfahren der BisDMATargets ist im weiteren Verlauf die Verwendung von PC bevorzugt worden, zumal die Eigenschaften beider Polymere insgesamt recht ähnlich sind. 


\subsection{Eigenschaften gemischter Polymerschichten}

Alle bisher vorgestellten Polymerschichten besitzen glatte Oberflächen. Jedoch zeigt sich, dass die PMMA-Schichten einen sehr niedrigen Elastizitätsmodul (einige MPa) besitzen im Vergleich zu den BisDMA- und PC-Schichten, welche einen Elastizitätsmodul von $6 \mathrm{GPa}$ aufweisen. In diesem Kapitel soll nun gezeigt werden, wie durch Kombination der verschiedenen Polymere die Eigenschaften der Schichten gezielt eingestellt werden können.

Für die Herstellung der Mischschichten aus PMMA und BisDMA bzw. PC befanden sich ein reines PMMA-Target und ein reines BisDMA- bzw. PC-Target in der UHVKammer, welche abwechselnd mit dem Laser beschossen wurden. Über die Anzahl der Laserpulse pro Target konnte der prozentuale Anteil des jeweiligen Polymers in den Mischschichten eingestellt werden.

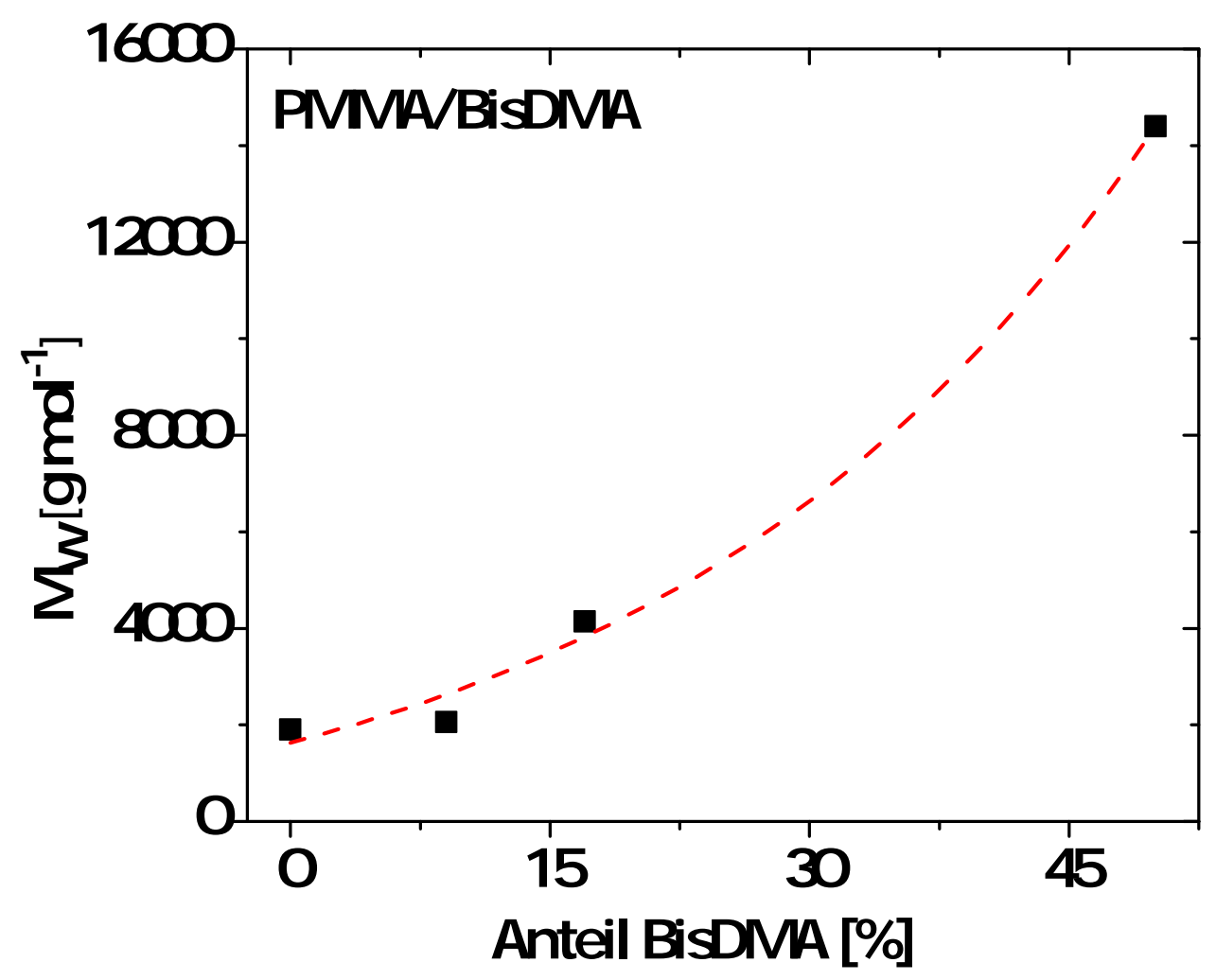

Abbildung 4.13: Abhängigkeit der mittleren Molmasse vom Anteil an BisDMA in den PMMA/BisDMA-Mischschichten. Es zeigt sich, dass mit zunehmendem Anteil an BisDMA die Kettenlänge der Mischschichten wächst. 
Zunächst war es von Interesse, die Veränderung der Kettenlänge mit steigendem BisDMA-Gehalt zu untersuchen. Dafür wurden SEC-Messungen an PMMA/BisDMAMischschichten mit unterschiedlichem BisDMA-Gehalt gemacht. In Abbildung 4.13 ist die Abhängigkeit der mittleren Molmasse vom Anteil BisDMA in den Mischschichten dargestellt. Es zeigt sich, dass mit zunehmendem Anteil an BisDMA die Kettenlänge der Mischschichten kontinuierlich ansteigt. Dabei steigt die mittlere Molmasse von anfangs $M_{w}=1,910^{3} \mathrm{~g} / \mathrm{mol}$ (reines PMMA) auf einen Wert von $M_{w}=1,4410^{4} \mathrm{~g} / \mathrm{mol}$ (PMMA/BisDMA 50 : 50), also um etwa eine Größenordnung. Dies ist ein erster Hinweis darauf, dass sich die beiden Polymere während der Deposition miteinander vernetzen.

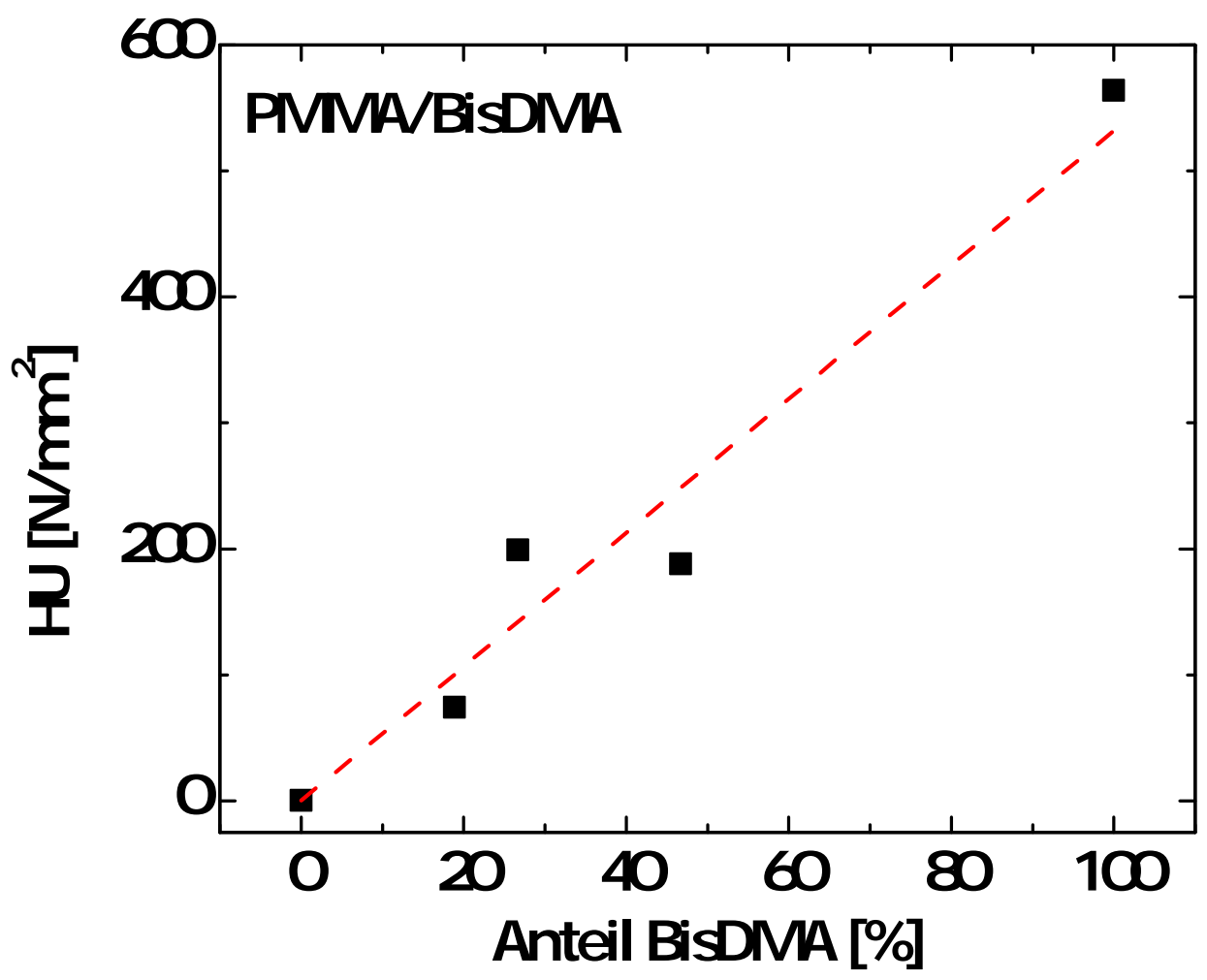

Abbildung 4.14: Abhängigkeit der Härte vom Anteil an BisDMA in den PMMA/BisDMAMischschichten. Es zeigt sich, dass mit zunehmendem Anteil an BisDMA die Härte der Mischschichten steigt (Zusammenarbeit mit Sara Lisa Jäckle).

Desweiteren wurden an diesen PMMA/BisDMA-Mischschichten Härtemessungen mittels Indentierung vorgenommen. In Abbildung 4.14 ist die Abhängigkeit der Härte vom Anteil an BisDMA in den Mischschichten aufgetragen. Mit zunehmendem 
Anteil an BisDMA steigt die Härte der Mischschichten linear an, was ein weiterer Hinweis für das Vernetzen der beiden Polymere ist. Dabei nimmt die Härte um drei Größenordnungen von $0,2 \mathrm{~N} / \mathrm{mm}^{2}$ auf $200 \mathrm{~N} / \mathrm{mm}^{2}$ zu bei gerade mal einem BisDMAAnteil von $50 \%$, und endet bei ca. $600 \mathrm{~N} / \mathrm{mm}^{2}$ für reine BisDMA-Schichten.

Weiterhin wurde der Elastizitätsmodul von PMMA/PC-Mischschichten untersucht. In Abbildung 4.15 ist die Abhängigkeit des E-Moduls vom Anteil an $\mathrm{PC}$ in den Mischschichten dargestellt. Hier zeigt sich, dass schon ein geringer Anteil von $1 \%$ PC dazu führt, dass der E-Modul der Mischschicht stark ansteigt und zwar vom MPaBereich in den GPa-Bereich. Bei etwa einem PC-Anteil von $30 \%$ läuft der E-Modul in eine Sättigung mit einem Wert von ca. 6 GPa. Der Anstieg des E-Moduls zeigt zum einen, dass sich auch PMMA und PC miteinander vernetzen, und zum anderen, dass sich mit den Mischschichten verschiedene E-Module gezielt einstellen lassen. Auch in der Literatur ist vielfach die Mischbarkeit von PMMA mit PC bestätigt worden [62,63], mit der Möglichkeit auch eine Steigerung des E-Moduls von PMMA/PC-Mischschichten zu erzielen [64].

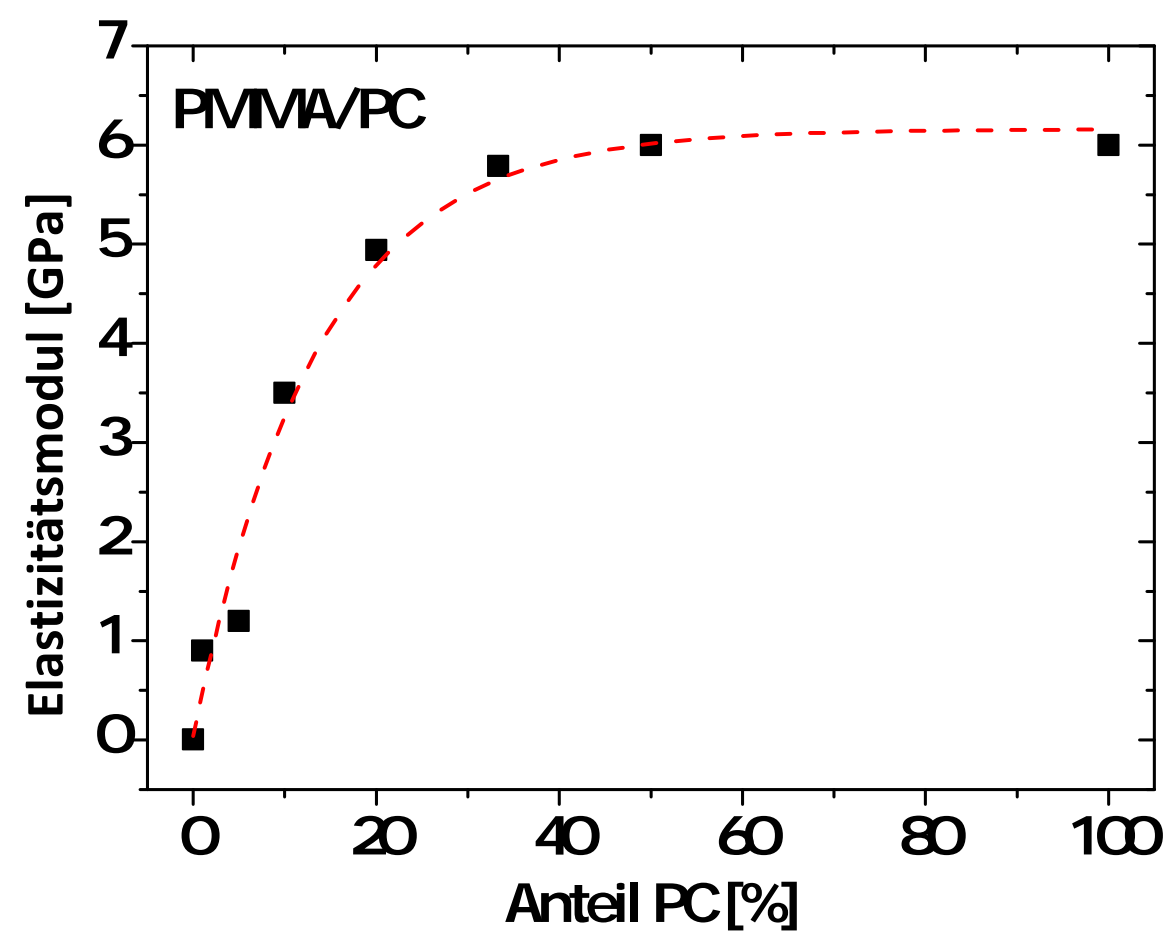

Abbildung 4.15: Elastizitätsmodul verschiedener PC/PMMA-Mischschichten in Abhängigkeit vom Mischungsverhältnis. Mit zunehmendem PC-Gehalt steigt der E-Modul der Schicht, so dass sich verschiedene E-Module gezielt einstellen lassen. 
Zusammenfassend konnte in diesem Kapitel gezeigt werden, dass durch die Kombination von PMMA mit den Polymeren BisDMA und PC es möglich ist die Kettenlängen und die mechanischen Eigenschaften (Härte, E-Modul) gezielt einzustellen. Dabei ist schon ein geringer Anteil an BisDMA bzw. PC ausreichend um die Kettenlänge und den E-Modul um ein Vielfaches zu steigern. Desweiteren lassen sich mit allen Polymeren glatte Schichten herstellen. 



\section{Spannungsinduzierte Wellenbildung im Polymer/Metall-System}

In diesem Kapitel werden die Grundlagen der spannungsinduzierten Wellenbildung im Polymer/Metall-System dargestellt. Es wird gezeigt, durch welche Parameter, wie zum Beispiel der Schichtdicke, die Wellenlänge auf der Oberfläche des Polymer/MetallSystems beeinflusst werden kann, und wie es gelingt die Wellen ganz gezielt anzuordnen. Zudem werden auch die Anfangsstadien des Wachstums der Metallschichten analysiert, um ein grundlegendes Verständnis des Entstehungsprozesses der Wellenbildung zu erhalten.

\subsection{Variation der $\mathrm{Cu}$-Schichtdicke}

In diesem Kapitel soll untersucht werden, in welchem Ausmaß die Wellenlänge von der deponierten $\mathrm{Cu}$-Schichtdicke abhängt, und ob bei allen Metallschichtdicken Wellenbildung stattfindet. Hierfür wurden auf 1,5 $\mu$ m dicken PMMA-Schichten unterschiedliche $\mathrm{Cu}$-Schichtdicken von 0,2 nm bis $300 \mathrm{~nm}$ deponiert und im AFM die sich einstellende Wellenlänge vermessen. In Abbildung 5.1 sind beispielhaft acht AFM-Aufnahmen gezeigt. Auf den ersten Blick lässt sich erkennen, dass alle Proben bis auf die 0,2 nm und $5 \mathrm{~nm}$ dicke Schicht Wellen aufweisen. Ab einer Schichtdicke von $10 \mathrm{~nm}$ ist zu beobachten, dass die Wellenlänge mit zunehmender Schichtdicke steigt. Deshalb wurde hier die Unterteilung in Frühstadien bis $10 \mathrm{~nm} \mathrm{Cu-Schichtdicke} \mathrm{und} \mathrm{Spätstadien} \mathrm{ab}$ $10 \mathrm{~nm}$ Schichtdicke vorgenommen, um das Verhalten hinsichtlich der Wellenlänge und Amplitude der Wellen in Abhängigkeit von der Metallschichtdicke systematisch zu untersuchen. Betrachtet man die Proben mit einer Cu-Schichtdicke von 0,8 nm, $1 \mathrm{~nm}$ und $300 \mathrm{~nm}$ genauer, so lässt sich erkennen, dass auf großen langwelligen Wellen noch eine Vielzahl kleiner Wellen existieren. Hier liegen somit zwei verschiedene Typen von Wellen vor, für die im Folgenden die Definition von Wellen der 1. und 2. Generation 

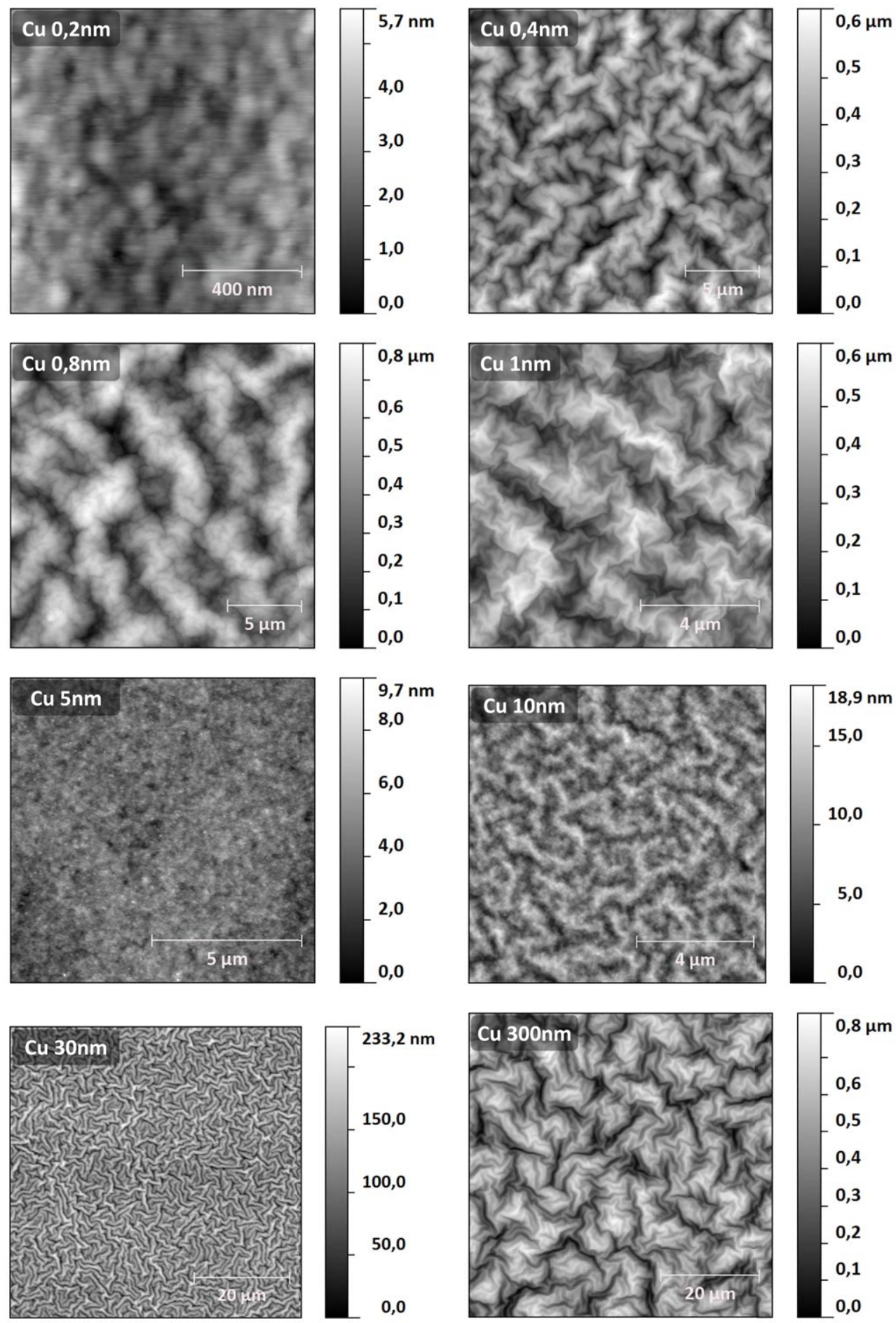

Abbildung 5.1: AFM-Aufnahmen von PMMA/Cu-Schichten mit unterschiedlichen Cu-Schichtdicken von $0,2 \mathrm{~nm}, 0,4 \mathrm{~nm}, 0,8 \mathrm{~nm}, 1 \mathrm{~nm}, 5 \mathrm{~nm}$ und $10 \mathrm{~nm}$ auf $1,5 \mu \mathrm{m}$ PMMA und $30 \mathrm{~nm}$ und $300 \mathrm{~nm}$ auf $500 \mathrm{~nm}$ PMMA. Bei genauerer Betrachtung fällt eine Cu-Schichtdicken abhängige Verteilung kleiner und großer Wellen auf. 
eingeführt wird. Dabei gehören die kleinen Wellen der 1. Generation, und die großen Wellen der 2. Generation an. Im Folgenden wird das gleichzeitige Auftreten beider Generationen auch als Doppelwelle bezeichnet.

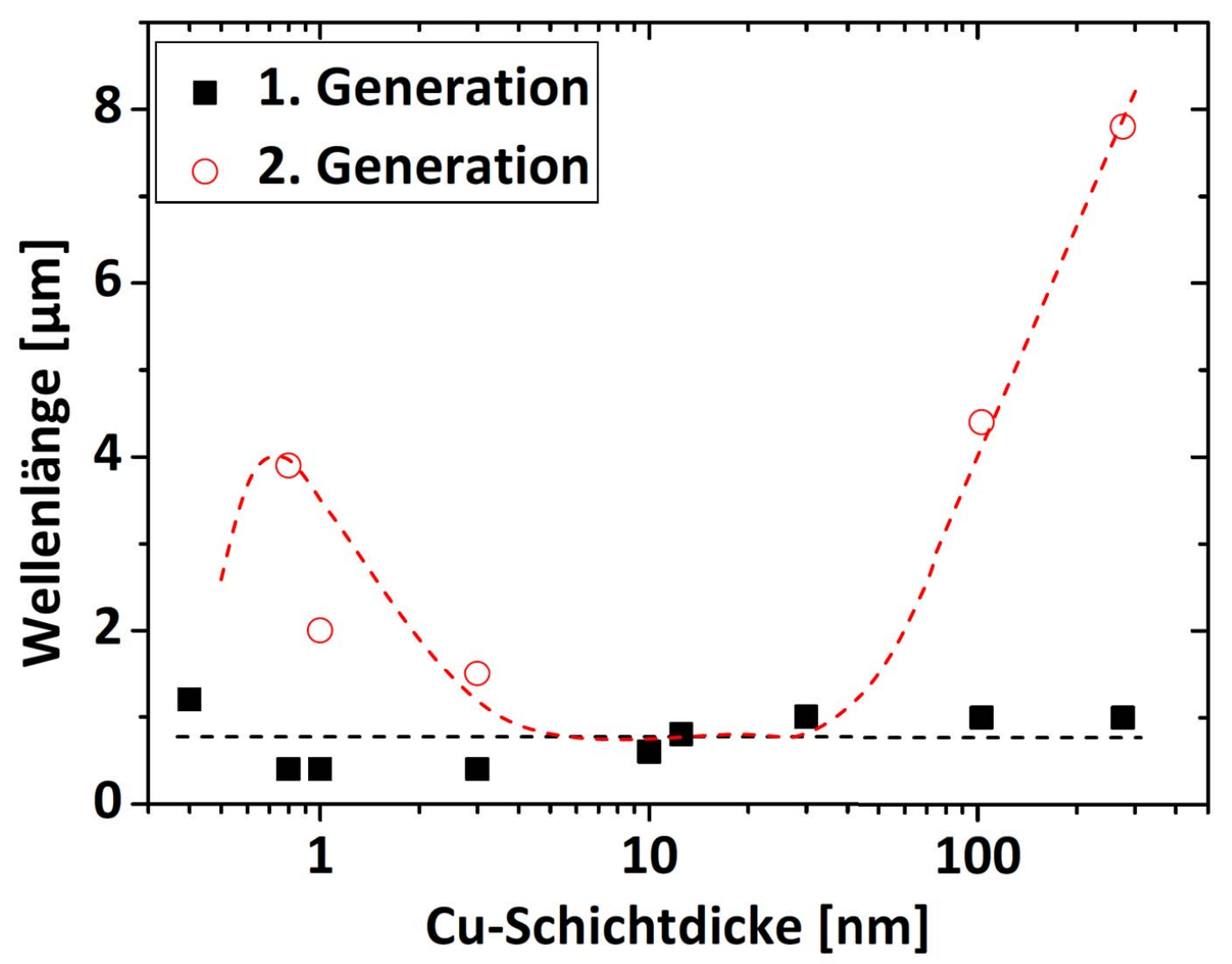

Abbildung 5.2: Abhängigkeit der Wellenlänge von der Cu-Schichtdicke zeigt die Ausbildung zweier Wellen-Generationen, die sich aus den AFM-Aufnahmen in ihrer Größe unterscheiden lassen.

Diese Ausbildung zweier Wellen-Generationen ist in Abbildung 5.2 mit der Abhängigkeit der Wellenlänge von der Cu-Schichtdicke graphisch dargestellt. Hier zeigt sich, dass anhand der unterschiedlichen $\mathrm{Cu}$-Schichtdicken die Wellenlänge direkt beeinflusst wird und sich somit gezielt über mehrere Mikrometer einstellen lässt. Betrachtet man zunächst die Frühstadien der Wellenbildung (unterhalb $10 \mathrm{~nm}$ ), so zeigt sich, dass die Wellenlänge der Wellen 2. Generation bei geringen $\mathrm{Cu}$-Schichtdicken von $0,4 \mathrm{~nm}$ bis $0,8 \mathrm{~nm}$ auf Werte von bis zu $4 \mu \mathrm{m}$ zunächst ansteigt. Die Existenz von Wellen sehr viel kleinerer Wellenlängen von $0,5 \mu \mathrm{m}$ bis $1 \mu \mathrm{m}$ (Wellen der 1 . Generation) im gleichen $\mathrm{Cu}$ Schichtdickenbereich führt zu der Ausbildung von Doppelwellen. Anschließend nimmt die Wellenlänge der Wellen 2. Generation wieder ab und zwar so stark, dass ab einer CuSchichtdicke von etwa $5 \mathrm{~nm}$ keine Wellen mehr vorhanden sind bzw. sich nicht mehr 
von der Rauigkeit der Probenoberfläche abgrenzen lassen. Erst in den Spätstadien der Wellenbildung zeigt sich ab einer $\mathrm{Cu}$-Schichtdicke von $10 \mathrm{~nm}$, dass sich wieder Wellen ausbilden, da sich mit zunehmender Schichtdicke die Wellenlänge der Wellen der 1. Generation minimal auf ca $1 \mu \mathrm{m}$ vergrößert. Ab einer Schichtdicke oberhalb von $30 \mathrm{~nm}$ treten wiederum die Wellen der 2. Generation auf. In den Spätstadien ist zu beobachten, dass die Wellenlänge der Wellen der 2. Generation sehr stark ansteigt auf einen Wert von bis zu ca. $8 \mu \mathrm{m}$ bei einer Cu-Schichtdicke von $300 \mathrm{~nm}$. Weiterhin ist bei den Doppelwellen zu beobachten, dass mit Ausbildung der 2. Generation, die Wellenlänge der 1. Generation konstant bei einer Wellenlänge von $1 \mu \mathrm{m}$ bleibt. Dies zeigt sich sowohl bei den Früh- als auch bei den Spätstadien der Wellenbildung.

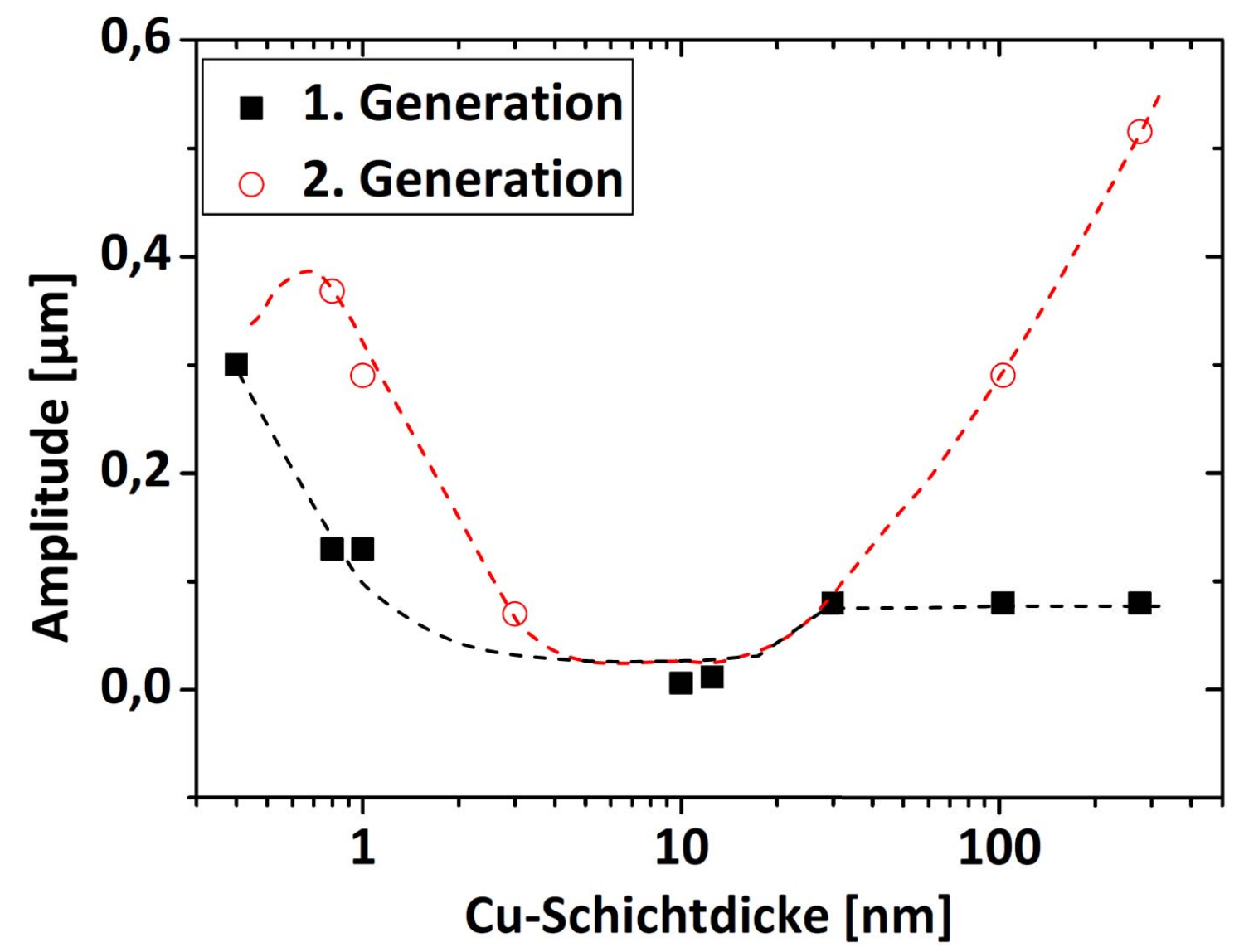

Abbildung 5.3: Abhängigkeit der Amplitude von der Cu-Schichtdicke zeigt die Ausbildung zweier Wellen-Generationen.

In Abbildung 5.3 ist die Amplitude, als weitere Kenngröße bei Wellen, in Abhängigkeit von der $\mathrm{Cu}$-Schichtdicke dargestellt. Für die Frühstadien der Wellenbildung $(<10 \mathrm{~nm})$ zeigt sich das gleiche charakteristische Verhalten wie bei der Wellenlänge. Zunächst steigt die Amplitude bei einer Cu-Schichtdicke zwischen 0,4 nm und 0,8 nm 
auf Werte von bis zu $0,4 \mu \mathrm{m}$ an. Dabei kommt es erneut zur Ausbildung von Doppelwellen. Anschließend nimmt die Amplitude in den Frühstadien mit steigender $\mathrm{Cu}$-Schichtdicke ab und verschwindet ab einer Cu-Schichtdicke von $5 \mathrm{~nm}$. In den Spätstadien ab einer Cu-Schichtdicke von $10 \mathrm{~nm}$ steigt die Amplitude wieder an, und es bilden sich erneut Doppelwellen aus. Oberhalb einer Cu-Schichtdicke von $30 \mathrm{~nm}$ steigert sich die Amplitude der Wellen der 2. Generation sukzessive bis auf einen Wert von ca. 0,5 $\mathrm{m}$ bei Erreichen einer Cu-Schichtdicke von $300 \mathrm{~nm}$. Auch hier ist zu beobachten, dass die Amplitude der Wellen der 1. Generation mit einem Wert von 0,1 $\mu$ m konstant bleibt, sofern sich die 2. Generation ausbildet.

Um alle hergestellten Proben in Bezug auf die ausgebildete Wellenstruktur besser vergleichen und interpretieren zu können, wurde im weiteren Verlauf die RMSRauigkeit aus den AFM-Aufnahmen bestimmt. Dies bietet den Vorteil, auch noch sehr kleine Wellenlängen auswerten zu können. Die RMS-Rauigkeit gibt die verschiedenen mittleren Höheninformationen einer Probe wieder.

Abbildung 5.4 zeigt den Kurvenverlauf der RMS-Rauigkeit in Abhängigkeit von der $\mathrm{Cu}$-Schichtdicke. In den Frühstadien der Wellenbildung bei sehr geringen $\mathrm{Cu}$ Schichtdicken bis ca. 0,8 nm ist zunächst ein starker Anstieg in der RMS-Rauigkeit zu erkennen, wie er auch schon bei der Wellenlänge und Amplitude der Wellen beobachtet wurde. Das Maximum, mit einer zunächst erstaunlich hohen RMS-Rauigkeit von etwa $150 \mathrm{~nm}$, befindet sich bei einer Cu-Schichtdicke von 0, $8 \mathrm{~nm}$, danach nimmt die Rauigkeit wieder ab und erreicht bei $10 \mathrm{~nm} \mathrm{Cu-Schichtdicke} \mathrm{eine} \mathrm{RMS-Rauigkeit} \mathrm{von} \mathrm{ca.} 3 \mathrm{~nm}$. Anschließend steigt die Rauigkeit wieder stark an auf einen Wert von ca. $150 \mathrm{~nm}$ bei $300 \mathrm{~nm}$ Cu-Schichtdicke.

Dieser Kurvenverlauf lässt sich wie folgt interpretieren. Wie aus Vorarbeiten von Störmer und Fähler bekannt, weisen laserdeponierte Metallschichten innere Spannungen auf [65]. Dies lässt sich dadurch erklären, dass die Teilchen bei der gepulsten Laserdeposition hohe kinetische Energien aufweisen. So besitzen die Ionen eine mittlere kinetische Energie von etwa $100 \mathrm{eV}$ und die deponierten Atome eine kinetische Energie von 5 bis $10 \mathrm{eV}$ [24]. Aufgrund der hohen kinetischen Energie der Ionen kommt es zur Implantation des Metalls in das Substrat (in diesem Fall: in die Polymerschicht), dies ist auch als „subsurface growth“ bekannt [38]. Dies hat zur Folge, dass sich das Material verdichtet und so ein Druckzustand entsteht. Dieser Mechanismus des Verdichtens wird auch als „shot peening“ (Strahlhämmern) oder „atomic peening“ bezeichnet. In den Arbeiten von Störmer wurden Druckspannungen für reine Fe-Schichten von etwa $1 \mathrm{GPa}$ und für homogen übersättigte $\mathrm{Fe}(\mathrm{Ag})$-Legierungen von 1 bis $2 \mathrm{GPa}$ beobachtet [65]. 


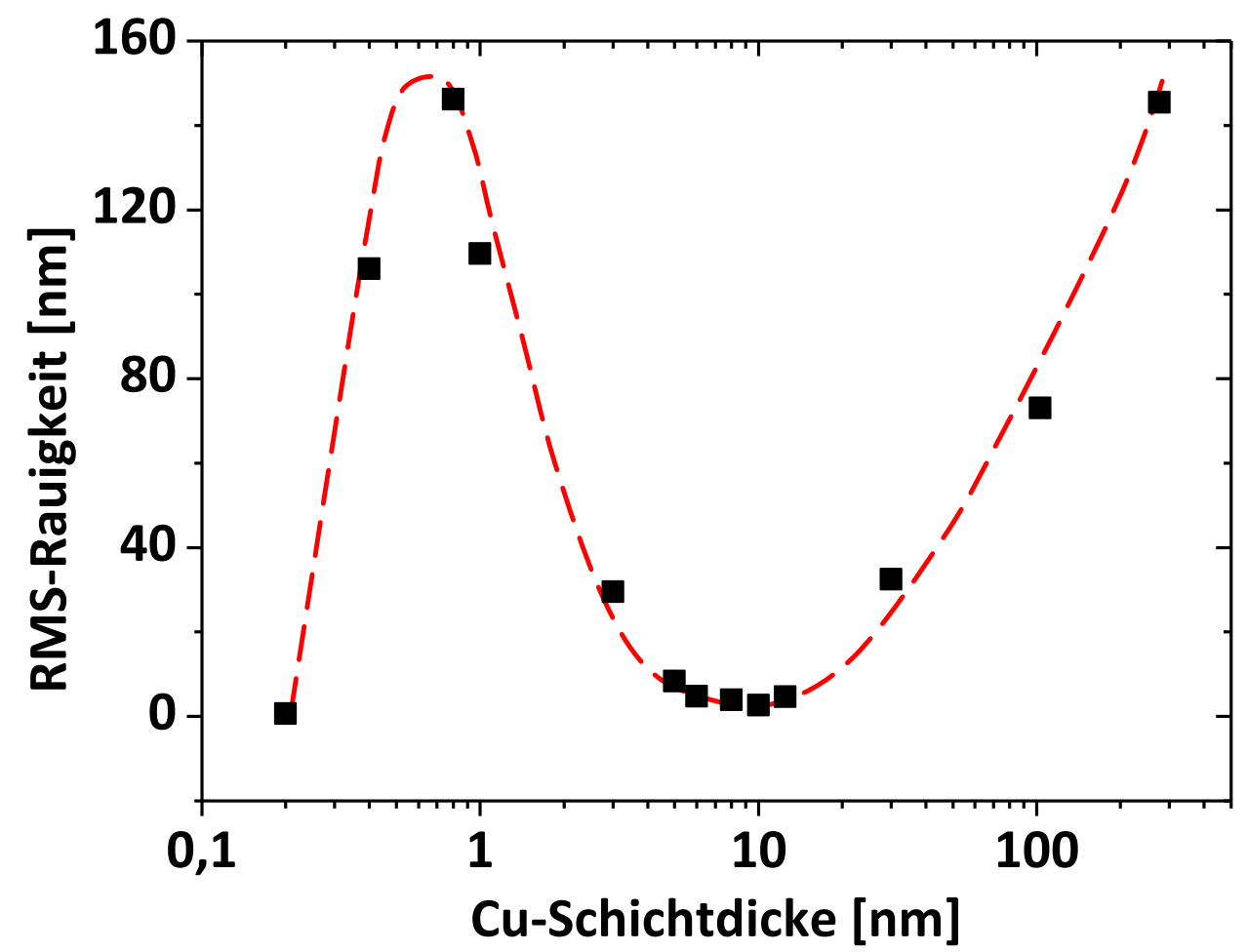

Abbildung 5.4: RMS-Rauigkeit in Abhängigkeit von der Cu-Schichtdicke. Die RMS-Rauigkeit durchläuft in den Frühstadien des Wachstums von $\mathrm{Cu}$ auf PMMA ein Maximum. Dies kann auf eine Vergrößerung des Gitterparameters in den kleinen Cu-Clustern zurückgeführt werden, was zu Druckspannungen in der Schicht führt. Der Abfall der Kurve lässt sich aufgrund von Zugspannungen, welche während der Koaleszenz benachbarter Cu-Cluster entstehen, erklären. Der erneute Anstieg in der Rauigkeit kann mit dem Verdichten des Materials in der geschlossenen Cu-Schicht durch die hohe Ionenenergie erklärt werden.

Diese inneren Spannungen können nur durch die Ausbildung von Wellen kompensiert werden, da die PMMA-Schicht auf dem Siliziumsubstrat durch eine gute Haftung gehalten wird, und somit eine Vergrößerung der Oberfläche nur durch die Ausbildung von Wellen kompensiert werden kann. Die Wellen sind somit ein guter Indikator dafür, ob Spannungen in der Metallschicht vorhanden sind. Der erste Anstieg in der Kurve lässt sich zum einen durch das „shot peening“ erklären, welches für Druckspannungen in der Metallschicht sorgt, welche sich durch das Verdichten des Materials aufgrund der hochenergetischen Teilchen ergibt.

Es kommt am Anfang allerdings noch zu einer weiteren Spannungskomponente, welche die Druckspannungen noch verstärkt. Dieser Effekt tritt nur in den Frühstadien des Wachstums auf, wenn der Metallgehalt an $\mathrm{Cu}$ noch so gering ist, dass isolierte Cluster im Polymer vorliegen. Denn bei Nanopartikeln ist der Gitterparameter gegen- 


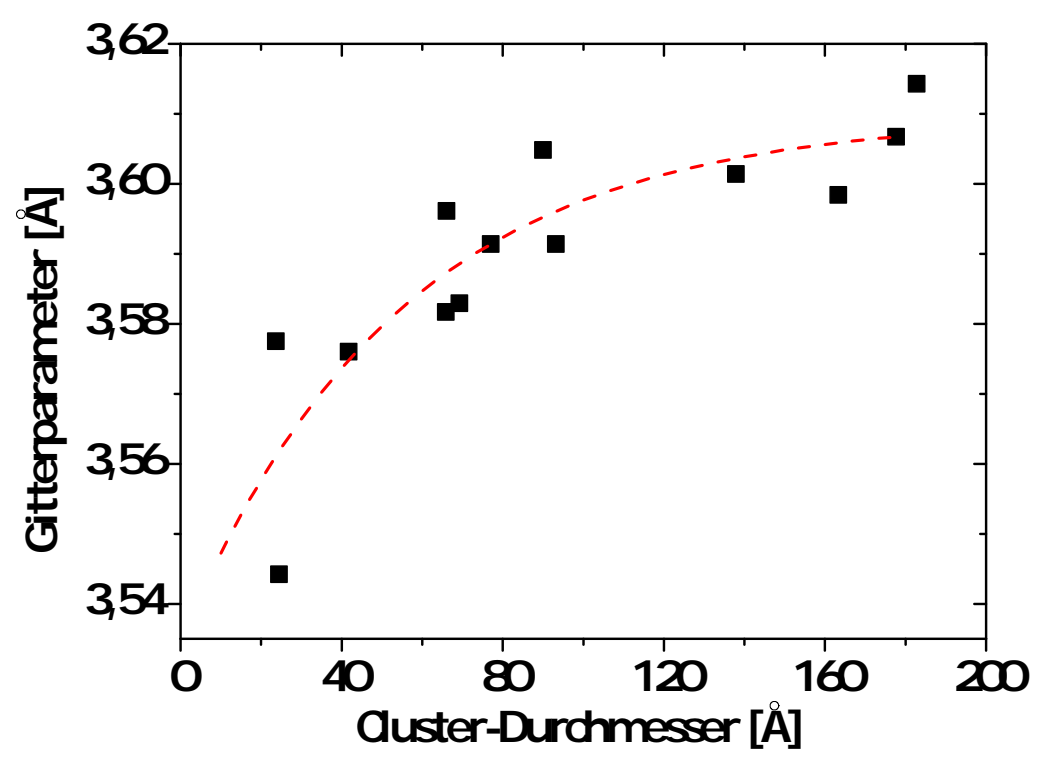

Abbildung 5.5: Gitterparameter in Abhängigkeit vom Cluster-Durchmesser am Beispiel Cu. In den Frühstadien des Clusterwachstums vergrößert sich bei steigendem Cu-Gehalt der Gitterparameter (Daten aus [66]).

über dem Wert des bulk-Materials stets verkleinert. Im Zuge des Clusterwachstums bei steigendem $\mathrm{Cu}$-Gehalt weitet sich somit das Kristallgitter im Cluster, wie folgende Graphik 5.5 zeigt. Die Gitterparameteraufweitung im Cu vom Dimer zum bulk-Material beträgt etwa $10 \%$ [67]. In der Literatur ist dieser Effekt auch als „capillary-induced growth stress" beschrieben [68-70].

Um diesen Einfluss der Gitterparameteraufweitung auf den anfänglichen starken Anstieg der RMS-Rauigkeit (bis 0, $8 \mathrm{~nm}$ ) besser einschätzen zu können, wurde folgendes Gedankenexperiment durchgeführt: man stelle sich zunächst einmal vor, dass der Effekt der Gitterparameteraufweitung die Cu-Schicht um etwa $10 \%$ (wie obig beschrieben) lateral verlängert. Da aber die Cu-Schicht auf der PMMA-Schicht und dem Si-Substrat sehr stark haftet, kann sie sich nicht lateral ausdehnen, was einem Druck von aussen gleichkommt. Die Cu-Schicht kann die dadurch entstehenden Druckspannungen nur in einer Vergrößerung der Oberfläche durch die Ausbildung von Wellen kompensieren. Die Wellen lassen sich dabei als Sinuskurve annähern (siehe Abbildung 5.6). Hierbei ist die Bogenlänge des Sinus im Vergleich zu einer geraden Linie der Länge $\lambda$ genau dann um ca. $10 \%$ länger, bei einem Amplituden-Wellenlängen-Verhältnis von:

$$
\frac{h}{\lambda}=\frac{1}{2 \pi} \quad 15,92 \%
$$




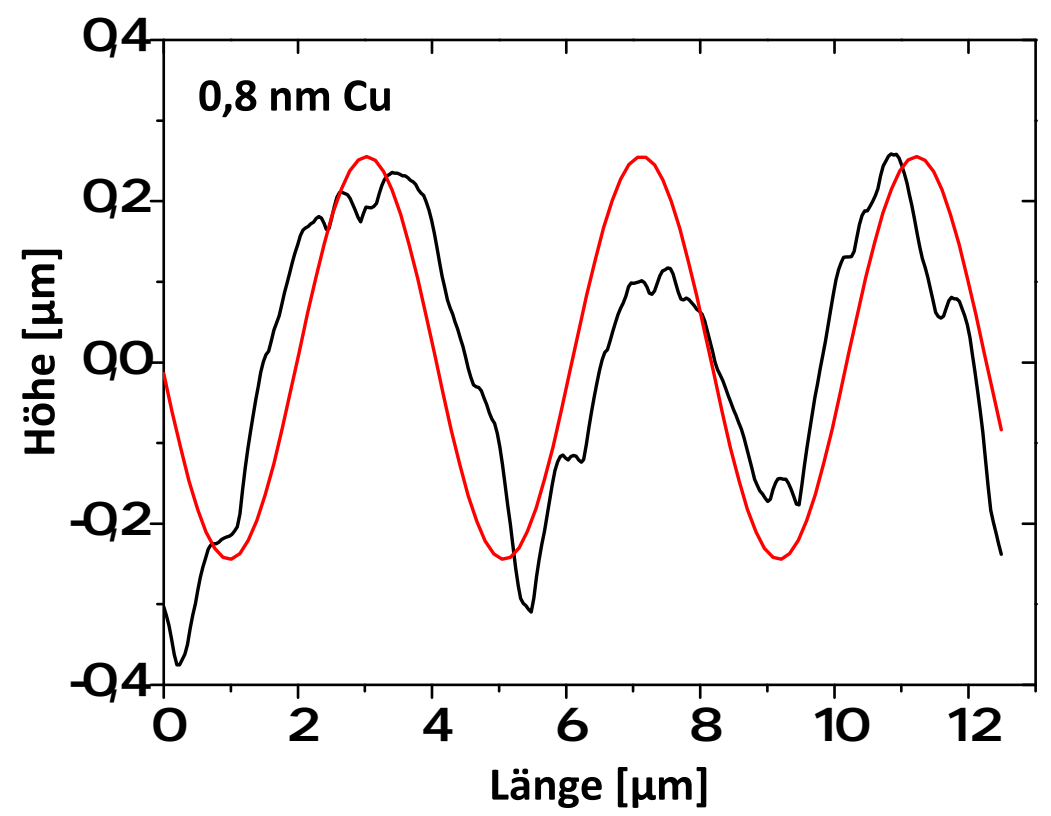

Abbildung 5.6: Höhenprofil der Probe mit einer Cu-Schichtdicke von 0, 8 nm. Die ausgebildeten Wellen lassen sich durch eine Sinus-Funktion beschreiben.

Für die Proben mit einer Cu-Schichtdicke von 0, $4 \mathrm{~nm}$ und 0, 8 nm wurde zur Überprüfung der Relation das Verhältnis aus Amplitude zur Wellenlänge gebildet. Hierbei ergab sich im Mittel ein Wert von $\left(\frac{h}{\lambda}\right)_{\text {exp }}=15,5$ 0,5\%. Die gemessenen Werte bestätigen also den Effekt der Gitterparameteraufweitung, welche in den Anfangswachstumstadien der $\mathrm{Cu}$-Schicht für die Ausbildung der großen Wellen sorgt. Hierbei sei allerdings noch bemerkt, dass die Darstellung sowohl der AFM-Bilder als auch des Höhenprofils in Abbildung 5.6 die Höhe bzw. Amplitude der Wellen stark überzeichnet. Deshalb ist in Abbildung 5.7 dasselbe Höhenprofil der Probe mit einer Cu-Schichtdicke von 0, $8 \mathrm{~nm}$ dargestellt, diesmal allerdings mit gleicher Skalierung der beiden Achsen. Dies verdeutlicht, dass die großen Wellen in den Anfangswachstumsstadien insgesamt recht flach sind.

Eine dazu entgegengesetzte Spannungskomponente kommt hinzu, wenn die CuCluster so weit gewachsen sind, dass sie miteinander koaleszieren. Dieser Effekt ist als „island zipping“ bekannt [71]. Durch die Koaleszenz zweier Cluster kommt es zur Ausbildung einer Korngrenze. Die treibenden Kräfte, welche versuchen werden den Spalt zu schließen, üben Zugspannungen auf die Unterlage, in diesem Fall auf die PMMA-Schicht, aus (siehe Abbildung 5.8). Diese Spannungskomponente wirkt den Druckspannungen entgegengesetzt, welche dauerhaft durch das "shot peening“ 


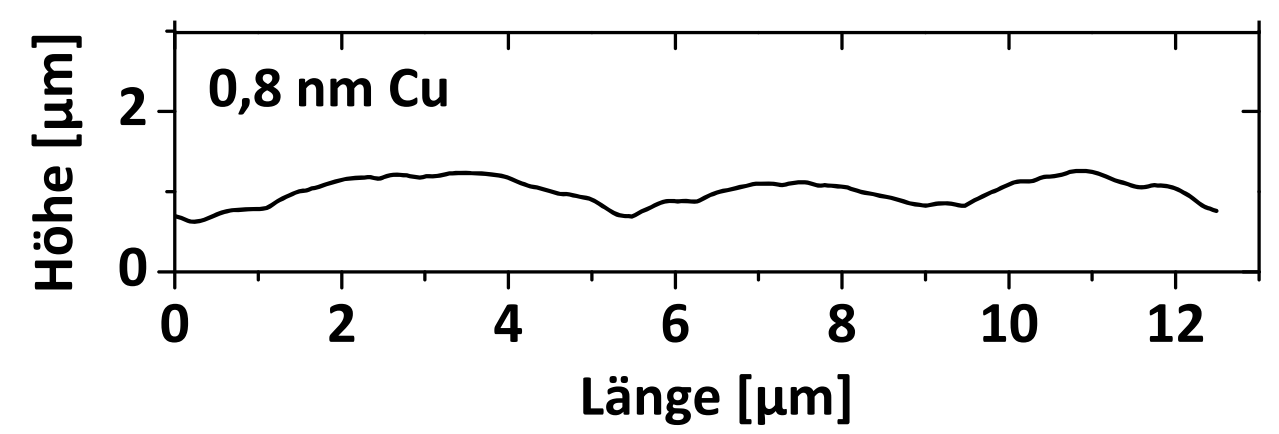

Abbildung 5.7: Höhenprofil der Probe mit einer Cu-Schichtdicke von 0, $8 \mathrm{~nm}$. Die gleiche Skalierung der beiden Achsen zeigt, dass die Wellen insgesamt sehr flach sind.

vorhanden sind. Dabei nehmen die Zugspannungen durch das „island zipping“ so lange zu bis die Cu-Schicht ab einer Cu-Schichtdicke von ungefähr $5 \mathrm{~nm}$ geschlossen ist. Dies beschreibt den Abfall der Kurve von 0,8 nm Cu-Schichtdicke bis $5 \mathrm{~nm}$, da in diesem Bereich die Zugspannungen gegenüber den Druckspannungen überwiegen. Seel et al. bestätigen dieses Auftreten von starken Zugspannungen. Ihren Untersuchungen zur Folge sind die Zugspannungen im Bereich der Perkolation, also demjenigen Wachstumsstadium, in dem die koaleszierten Cluster netzwerkartige Strukturen ausgebildet haben, besonders groß [72]. Wenn die Cu-Schicht bei einer Schichtdicke von $5 \mathrm{~nm}$ geschlossen ist, kommt es in einem kleinen Schichtdickenbereich zum Gleichgewicht der beiden Spannungskomponenten. In diesem Bereich gleichen sich die Zug- und Druckspannungen fast aus, weshalb es auch zum Verschwinden der Wellen kommt.
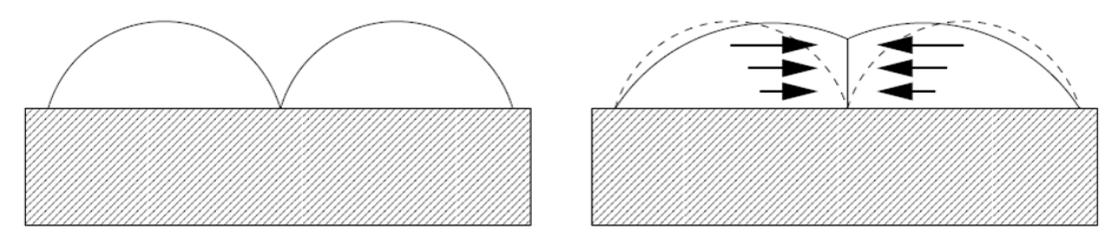

Abbildung 5.8: Schematische Darstellung der Koaleszenz zweier Kugelkappen auf einem Substrat (Daten aus [73]). Treibende Kräfte, welche versuchen den Spalt zwischen den Clustern zu schließen (, ,island zipping“), üben Zugspannungen auf das Substrat aus.

Mit Erreichen des Minimums (ab ca. $5 \mathrm{~nm}$ ) ist die $\mathrm{Cu}$-Schicht komplett geschlossen. Dies bedeutet, dass die Zugspannungen, welche durch die Nukleation der CuCluster hervorgerufen wurden, nun ihren maximalen Spannungswert erreicht haben. Beim weiteren Wachstum der $\mathrm{Cu}$-Schicht überwiegen nun wieder hauptsächlich die Druckspannungen, welche durch das "shot peening“ hervorgerufen werden. Somit 
nimmt sowohl die Rauigkeit als auch die Wellenlänge mit zunehmender $\mathrm{Cu}$-Schicht kontinuierlich zu.

Durch die Interpretation des Kurvenverlaufs aus Abbildung 5.4 konnte also gezeigt werden, dass es zu einem Spannungswechsel von Druck- zu Zug- und wiederum zu Druckspannungen in den Anfangswachstumsstadien der Cu-Schicht kommt. Dieser Spannungswechsel wurde auch schon in Vorarbeiten anhand von Spannungsmessungen an laserdeponierten $\mathrm{Fe} / \mathrm{MgO}-\mathrm{Multischichten} \mathrm{beobachtet} \mathrm{[68].}$

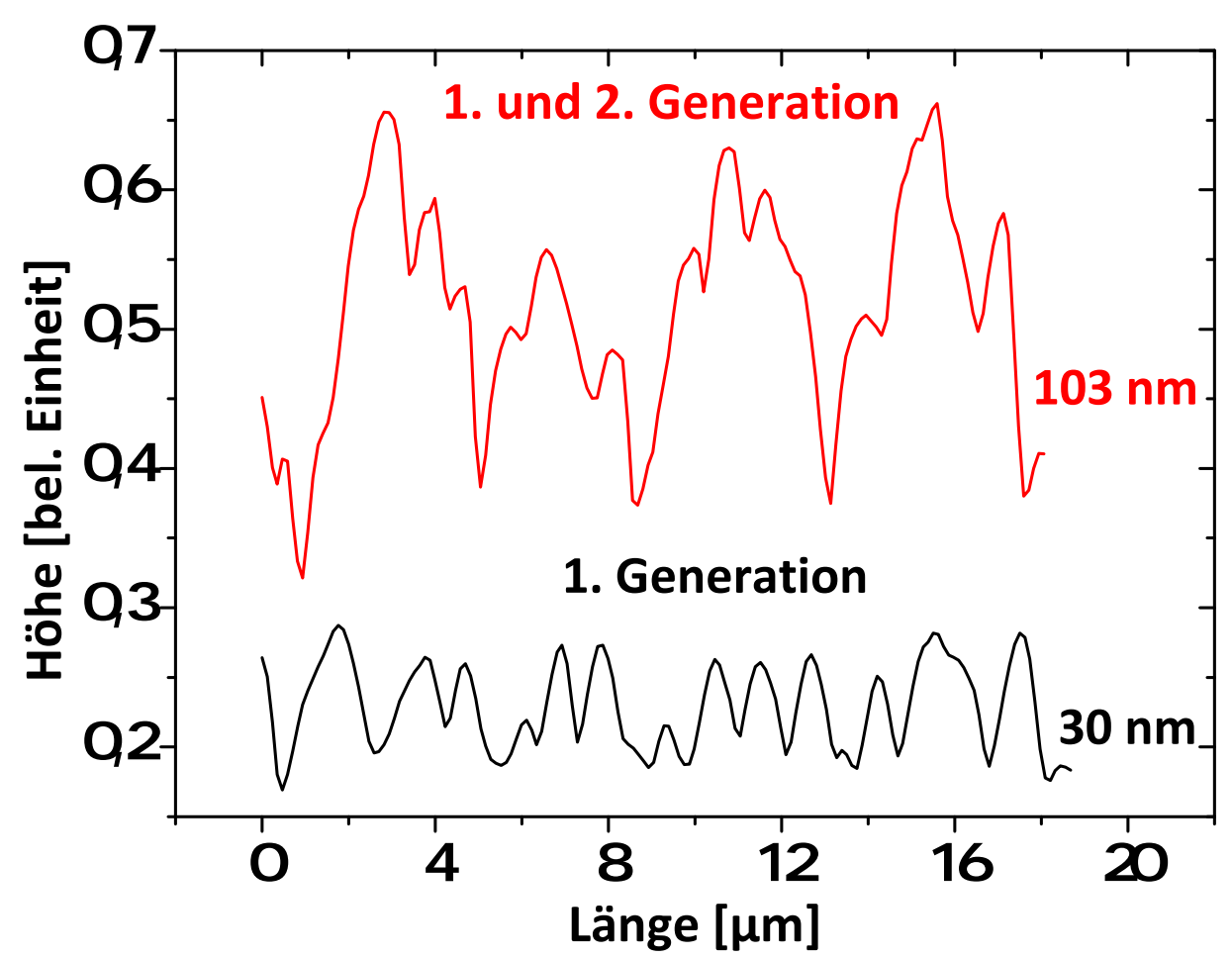

Abbildung 5.9: Höhenprofile zweier Proben mit $30 \mathrm{~nm}$ und $103 \mathrm{~nm}$ Cu-Schichtdicke auf $500 \mathrm{~nm}$ PMMA. Während bei der $30 \mathrm{~nm}$ dicken $\mathrm{Cu}$-Schicht lediglich kleine Wellen der 1. Generation auftreten, zeigen sich Wellen der 1. und 2. Generation bei dickeren Schichten, in denen erhöhte Druckspannungen vorliegen sollten.

Die Ausbildung zweier Generationen an Wellen lässt sich wie folgt interpretieren. Sowohl die Wellenlänge als auch die Amplitude der 1. Generation bleiben konstant, sofern sich die 2. Generation der Wellen ausbildet. Die 1. Generation bildet die kleinen Wellen aus, welche bei der 2. Generation auf den großen Wellen weiterexistieren, sich aber in ihrer Form und Größe nicht mehr verändern. Dies ist in Abbildung 5.9 anhand von Höhenprofilen zweier Proben dargestellt. Hier zeigt die Probe mit einer $\mathrm{Cu}$-Schichtdicke von $30 \mathrm{~nm}$ Wellen der 1. Generation und die Probe mit einer $\mathrm{Cu}-$ 
Schichtdicke von ca. $100 \mathrm{~nm}$ zusätzlich Wellen der 2. Generation. Es lässt sich gut erkennen, dass die kleinen Wellen der 1. Generation auf den großen Wellen der 2. Generation weiterbestehen. Dies ist ein Hinweis darauf, dass sich die 1. Generation als erstes ausbildet. Weiterhin ist zu beobachten, dass sich die 2. Generation immer dann ausbildet, wenn die Spannungen in der Schicht stark zunehmen. So ist in den Frühstadien eine große Spannung in den Cu-Schichten vorhanden aufgrund des „shot peening" und des Effekts der Gitterparameteraufweitung. Auch in den Spätstadien steigt mit zunehmender Cu-Schichtdicke aufgrund des Verdichtens von Schichtmaterial die Druckspannung in den Schichten, was ebenfalls zur Ausbildung von Doppelwellen führt.

Zusammenfassend konnte gezeigt werden, dass die Ausbildung von Wellen im Polymer/Metall-System ein guter Indikator ist, um die Spannungen in der Metallschicht $\mathrm{zu}$ verfolgen und $\mathrm{zu}$ verstehen. Die verschiedenen Spannungskomponenten haben großen Einfluss auf die Wellenbildung und machen es sogar möglich, die Wellen beliebig in ihrer Größe einzustellen. Es bilden sich abhängig vom Spannungszustand im Polymer/Metall-System unterschiedliche Generationen an Wellen aus, die sich in ihrer Größe voneinander unterscheiden.

\subsection{Variation der PMMA-Schichtdicke}

Nachdem gezeigt wurde, wie die Wellenlänge von der $\mathrm{Cu}$-Schichtdicke abhängt, soll nun untersucht werden, welchen Einfluss die Variation der PMMA-Schichtdicke auf die Wellenlänge hat. Hierfür wurden mehrere Schichten mit variabler PMMASchichtdicke und konstanter Cu-Schichtdicke von $50 \mathrm{~nm}$ auf Si-Substraten hergestellt. Anschließend wurden die sich einstellenden Wellenlängen mit dem AFM vermessen. In Abbildung 5.10 sind beispielhaft AFM-Aufnahmen von vier Proben mit PMMA-

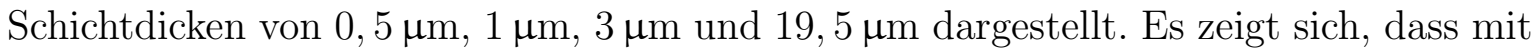
zunehmender PMMA-Schichtdicke die Wellenlänge zunimmt und sich außerdem, wie im vorangegangenem Kapitel beschrieben, Wellen beider Generationen ausbilden.

Die Auftragung der Wellenlänge gegenüber der PMMA-Schichtdicke ist in Abbildung 5.11 dargestellt. Ab einer PMMA-Schichtdicke von $3 \mu \mathrm{m}$ kommt es zur Ausbildung von Wellen der 1. und 2. Generation. Die Wellen der 1. Generation weisen eine konstante Wellenlänge von $2 \mu \mathrm{m}$ auf, sofern sich die 2. Generation ausbildet. Bis zu einer PMMASchichtdicke von $20 \mu \mathrm{m}$ steigt die Wellenlänge der 2. Generation auf einen Wert von 

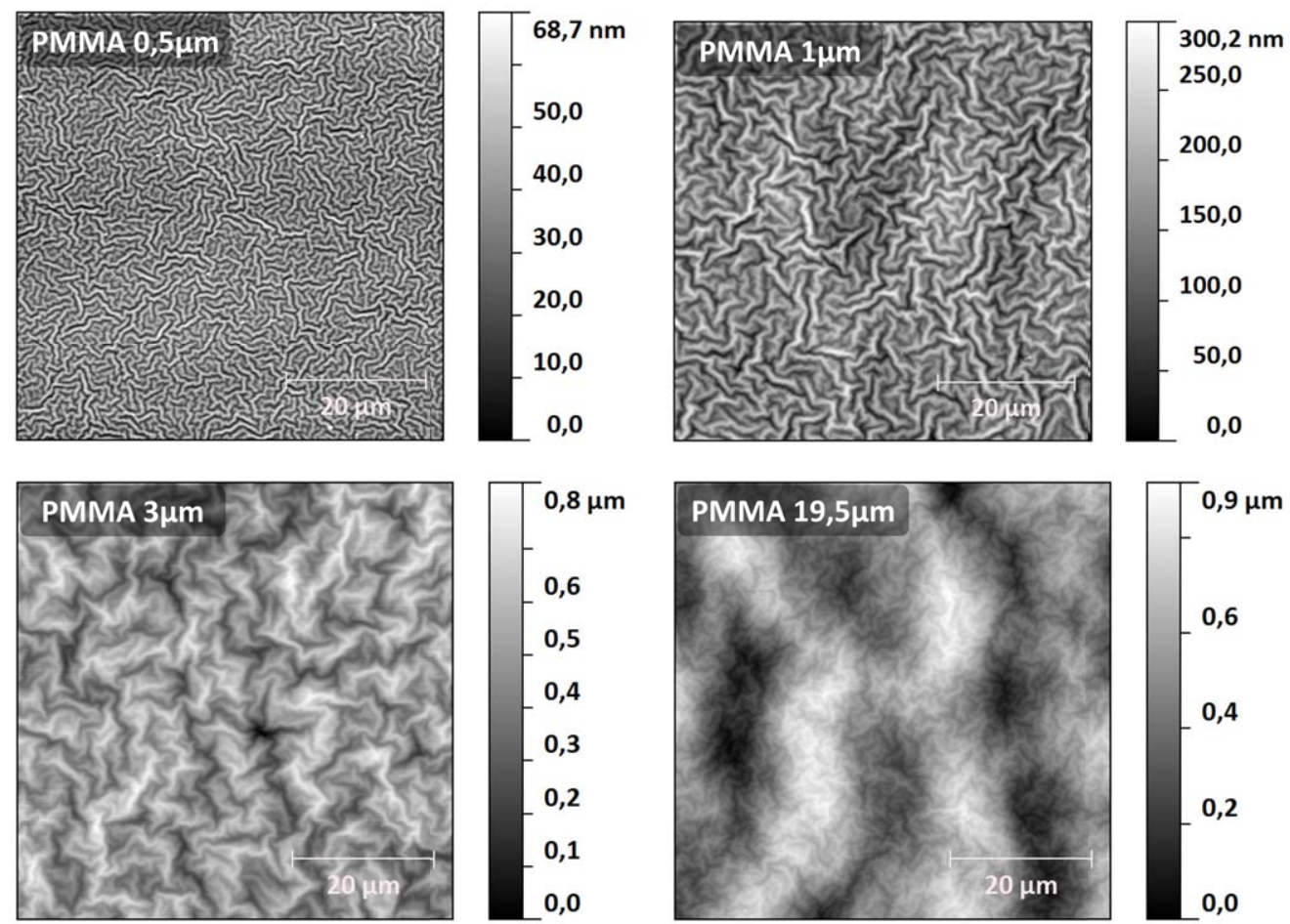

Abbildung 5.10: AFM-Aufnahmen von vier PMMA/Cu-Proben mit unterschiedlicher PMMASchichtdicke $(0,5 \mu \mathrm{m}, 1 \mu \mathrm{m}, 3 \mu \mathrm{m}$ und 19,5 $\mathrm{m})$ und konstanter Cu-Schichtdicke von $50 \mathrm{~nm}$. Die Aufnahmen zeigen verstärkt die Zunahme der Wellenlänge mit ansteigender PMMA-Schichtdicke. Für sehr dicke PMMA-Schichten von 19,5 $\mathrm{m}$ sind Wellen zweier Generationen auszumachen.

$23 \mu \mathrm{m}$ an. Dies zeigt zum einen, dass die Wellenlänge durch die Variation der PMMASchichtdicke leicht zu beeinflussen ist, und somit andererseits ganz gezielt über mehrere Mikrometer eingestellt werden kann. Ein Grund für die Beeinflussung der Wellenlängen bei ganz dünnen PMMA Schichten ist das verwendete Si-Substrat. Je dünner die PMMA-Schicht, desto stärker ist der Einfluss des Si-Substrates auf die Cu-Schicht. Erst ab einer PMMA-Schichtdicke von $20 \mu \mathrm{m}$ ist der Einfluss des Si-Substrates für die $\mathrm{Cu}-$ Schicht verschwunden, da ab dieser Schichtdicke die Wellenlänge in eine Sättigung mit einem Wert von ca. $23 \mu \mathrm{m}$ geht.

Die verwendeten Si-Substrate besitzten einen Elastizitätsmodul von $150 \mathrm{GPa}$. Um zu überprüfen, ob der Übergang zu einer konstanten Wellenlänge auch mit einer niedrigeren PMMA-Schichtdicke möglich ist, wurde ein Substrat mit einem kleineren Elastizitätsmodul als der des Si-Substrates ausgewählt. Hierfür wurde eine PMMAPlatte benutzt, welche sonst als Targetmaterial dient und einen Elastizitätsmodul von 4 GPa besitzt. Darauf wurden Doppelschichtsysteme mit variabler PMMA-Schichtdicke und konstanter Cu-Schichtdicke von $30 \mathrm{~nm}$ deponiert. Die AFM-Aufnahmen in Ab- 


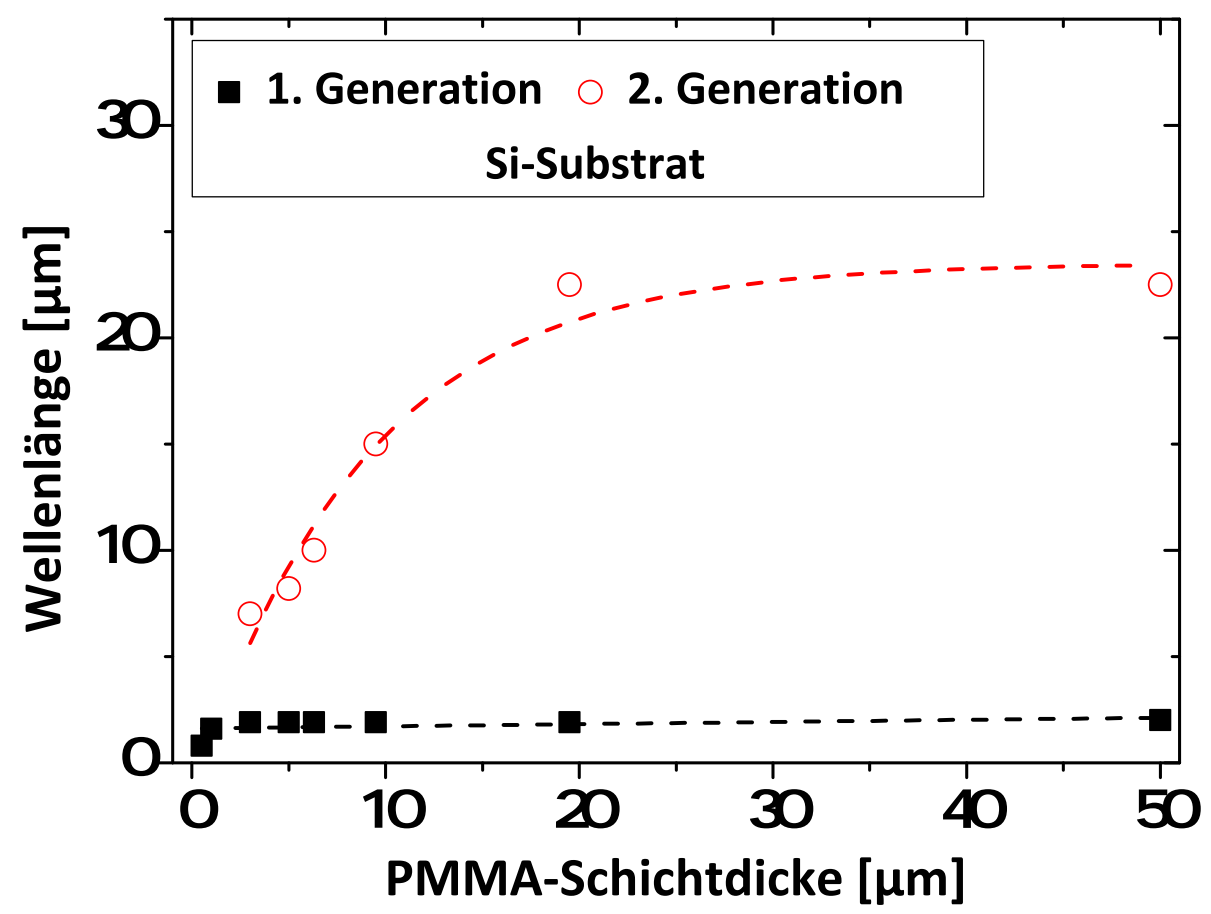

Abbildung 5.11: Abhängigkeit der Wellenlänge von der PMMA-Schichtdicke. Die Cu-Schichtdicke betrug bei allen Proben $50 \mathrm{~nm}$, die Schichten wurden auf einem Si-Substrat deponiert. Der drastische Anstieg der Wellenlänge von Wellen der 2. Generation zeigt eine Möglichkeit zur Variation der Wellenlänge über mehrere Mikrometer. Der Übergang der Wellenlängen-Werte in eine Sättigung von ca. $23 \mu \mathrm{m}$ spricht für ein Verschwinden des Substrateinflusses.

bildung 5.12 zeigen, dass sich die Wellenlänge bei großen PMMA-Schichtdicken ab ca. $3 \mu \mathrm{m}$ kaum noch verändert. Dies zeigt sich deutlich auch in der folgende Graphik 5.13, welche die Wellenlänge in Abhängigkeit der PMMA-Schichtdicke darstellt. Der Kurvenverlauf ähnelt der vorherigen Kurve (siehe Abbildung 5.11) für die Schichten auf dem Si-Substrat, allerdings mit dem entscheidenen Unterschied, dass der Übergang in die Sättigung bereits schon ab einer PMMA-Schichtdicke von $6 \mu \mathrm{m}$ erreicht wird. Hier zeigt sich ebenfalls, dass die Wellen der 1. Generation eine konstante Wellenlänge knapp unterhalb von $1 \mu \mathrm{m}$ aufweisen, sofern sich die 2 . Generation ausbildet.

Das sich Einstellen einer konstanten Wellenlänge der 2. Generation, welches durch Auslöschung des Substrateinflusses bei großen PMMA-Schichtdicken auftritt, ermöglicht nun eine Berechnung bzw. erste Abschätzung des Elastizitätmoduls $E_{P}$ der laserdeponierten PMMA-Schicht. Dazu soll nun im Folgenden nach der SIEBIMMMethode die Formel (2.17) aus Kapitel 2 verwendet werden. 

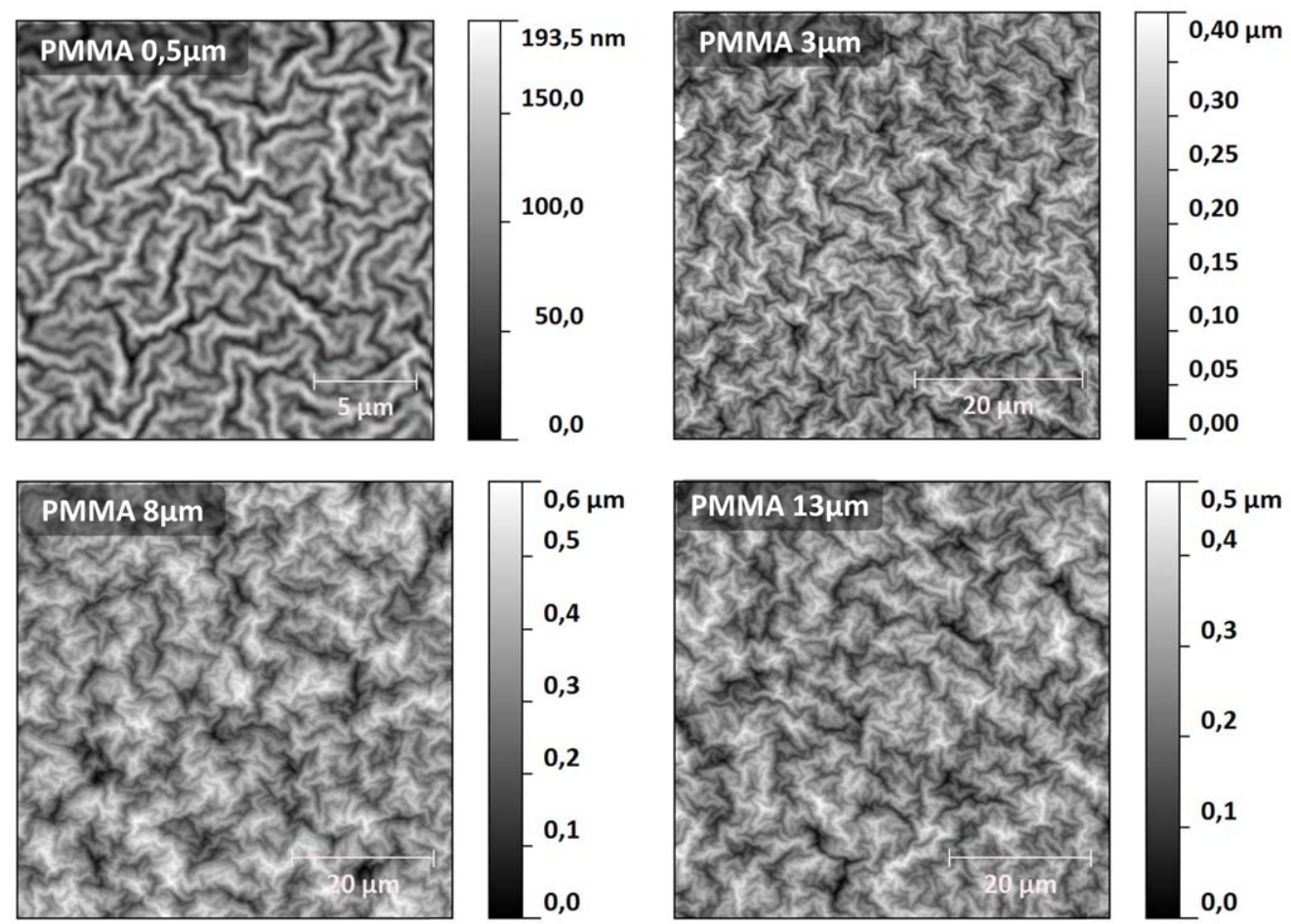

Abbildung 5.12: AFM-Aufnahmen von vier Proben mit einer konstanten Cu-Schichtdicke von $30 \mathrm{~nm}$ und variabler PMMA-Schichtdicke $(0,5 \mu \mathrm{m}, 3 \mu \mathrm{m}, 8 \mu \mathrm{m}$ und $13 \mu \mathrm{m})$ auf einem PMMA-Substrat. Im Vergleich zum Si-Substrat stellt sich hier viel schneller ein Sättigungswert in der Wellenlänge ein.

$$
E_{P}=\frac{E_{M}}{3}\left(\frac{\lambda}{2 \pi h_{M}}\right)^{-3}
$$

Hierbei beträgt der Elastizitätsmodul für die $\mathrm{Cu}$-Schicht $E_{M}=115 \mathrm{GPa}$, wie in Zusammenarbeit mit Inga Knorr mit Hilfe des Nanoindenters an mehreren CuSchichten vermessen wurde [74]. Bei diesem Probensystem (Schichtdicken: $h_{M}=$ $30 \mathrm{~nm}, h_{P}=13 \mu \mathrm{m}$ und einer Wellenlänge von $\left.\lambda=4,4 \mu \mathrm{m}\right)$ ließ sich der E-Modul Wert für die PMMA-Schicht auf $E_{P}=3 \mathrm{MPa}$ abschätzen. Wie schon in Kapitel 4.1 erwähnt, haben bisherige obere Abschätzungen mittels AFM-Indentermessungen auf einen Elastizitätsmodul im unteren MPa-Bereich hingewiesen. Vergleicht man den hier bestimmten Wert von $3 \mathrm{MPa}$, mit Werten aus der Literatur für Polymere mit einem sehr niedrigen Elastizitätsmodul, so lassen sich die laserdeponierten PMMASchichten mit Elastomeren und Gummi vergleichen (siehe Tabelle 5.1). Weiterhin zeigt der Vergleich mit dem E-Modul vom PMMA-Target (4 GPa), dass die durch Laserdeposition resultierende Kettenverkürzung einen dramatischen Einfluss auf den E-Modul der PMMA-Schicht hat. 


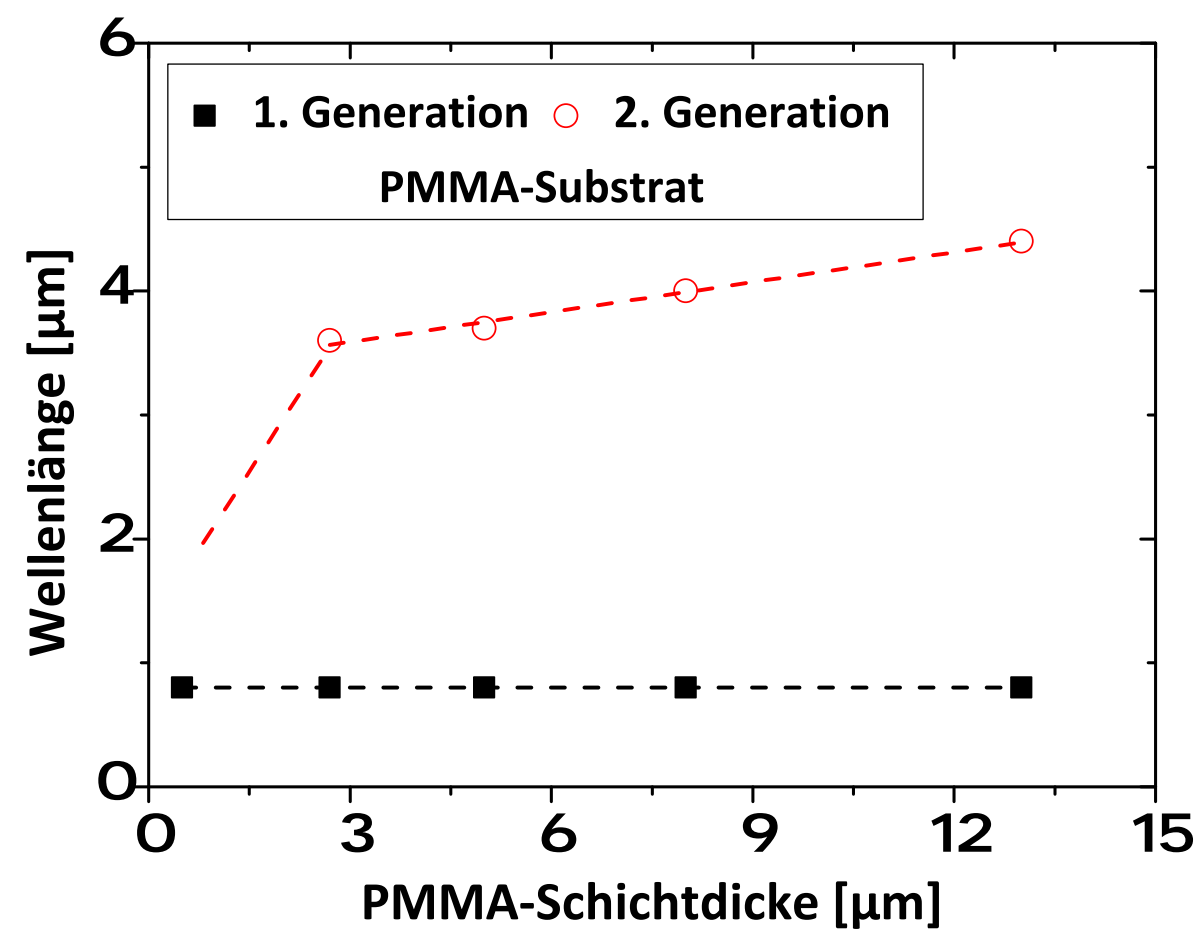

Abbildung 5.13: Abhängigkeit der Wellenlänge von der PMMA-Schichtdicke. Die Cu-Schichtdicke betrug bei allen Proben $30 \mathrm{~nm}$ und die Schichten wurden auf einem PMMA-Substrat deponiert. Es zeigt sich ein ähnlicher Kurvenverlauf wie auf einem Si-Substrat (s. Abb. 5.11), aber mit dem entscheidenen Unterschied, dass der Übergang in die Sättigung bei weitaus kleineren PMMASchichtdicken im Bereich von ca. $6 \mu \mathrm{m}$ erreicht wird.

Die AFM-Indentermessungen (in Abb. 4.7 und 4.8) haben zusätzlich gezeigt, dass sich das PMMA auf kurzen Zeitskalen wie ein elastischer Festkörper verhält und auf langen Zeitskalen $(>1 \mathrm{~s})$ wie eine viskose Flüssigkeit. Betrachtet man die Prozesse beim Aufwachsen der Cu-Schicht, so konnte Fähler anhand von Flugzeitmessungen an laserdeponierten Schichten zeigen, dass das Material innerhalb von $1 \mu$ s auf dem Substrat auftrifft [24]. Weiterhin finden beim Wachstum einer dünnen Schicht verschiedene Prozesse auf unterschiedlichen Zeitskalen statt. Auf sehr kurzen Zeitskalen, in der Größenordnung weniger Gitterschwingungen ( $10^{13}$ bis wenige $10^{12} \mathrm{~s}$ ), kommt es zu Umordnungsprozessen bei denen das auftreffende Atom seine kinetische Energie abgibt und mit dem Substrat in ein thermisches Gleichgewicht übergeht [75]. Auf längeren Zeitskalen ( $\left.>10^{11} \mathrm{~s}\right)$ finden Prozesse wie Diffusion, Keimbildung und Clusterwachstum statt. Dies lässt vermuten, dass die Ausbildung der Wellen in einem 


\begin{tabular}{|l|r|}
\hline Material & E-Modul $[\mathrm{MPa}]$ \\
\hline \hline Gelatine & $0,02[61]$ \\
\hline PVA gel & $0,005[76]$ \\
\hline Polyisopren Gummi & $1-3[77]$ \\
\hline PVC & $1000-3500$ \\
\hline Pyreflex PU & $10-40[77]$ \\
\hline Elastomere & $10-100$ \\
\hline PDMS & $1,5-2[78]$ \\
\hline
\end{tabular}

Tabelle 5.1: Elastizitätsmodule verschiedener Polymere mit niedrigem Elastizitätsmodul

Zeitraum stattfindet, in dem die PMMA-Schicht wie ein elastischer Festkörper reagiert, und somit der bestimmte E-Modul von $3 \mathrm{MPa}$ in der richtigen Größenordnung liegt.

Diese durch AFM-Indentermessungen bestätigte neue Messmethode zur Bestimmung der Elastizitätsmoduln sehr weicher Materialien hat sich auch in der Literatur etabliert. Insbesondere die Gruppe um Stafford et al. haben gezeigt, dass die Messung von EModuln an Polymeren mit Hilfe der SIEBIMM-Methode möglich ist [78]. Desweiteren konnte sie zeigen, dass die Bestimmung des E-Moduls für Polymere mit Hilfe der SIEBIMM-Methode ähnliche Ergebnisse liefert, wie durch Indentierung gemessen [13]. Dies verdeutlicht die Genauigkeit dieser Messmethode. Nolte et al. zeigen, dass mit SIEBIMM auch Multischichten aus Polymeren vermessen werden können [79]. Der Vorteil dieser Methode im Vergleich zur Nanoindentierung ist zum einen, dass auch sehr weiche Schichten vermessen werden können, und zum anderen, dass die Schichten sehr dünn sein können.

Insgesamt konnte gezeigt werden, dass die Wellenlänge neben der Metallschichtdicke (s. Kap. 5.1) auch von der PMMA-Schichtdicke abhängt und sich über mehrere Mikrometer einstellen lässt. Desweiteren ist die Wellenlänge auch abhängig vom verwendeten Substrat und zeigt bei einem Substrat mit geringerem E-Modul einen schnelleren Übergang in eine konstante Wellenlänge, welche sich ohne Substrateinfluss ausbilden. Dies ließ die Möglichkeit zu den E-Modul der PMMA-Schicht mit ca. $3 \mathrm{MPa}$ über die SIEBIMM-Methode zu berechnen. Im Vergleich zur Literatur und durch AFM-Indentermessungen konnte dieser Wert bestätigt werden und zeigt, dass die laserdeponierten PMMA-Schichten damit einen Elastizitätsmodul aufweisen, welchen auch Elastomere und Gummi zuzuordnen ist. 


\subsection{Einfluss einer Ar-Atmosphäre}

In Kapitel 5.1 konnte gezeigt werden, dass die Wellen ein guter Indikator für den jeweils vorliegenden Spannungszustand in den PMMA/Cu-Schichtsystemen sind. Aus Vorarbeiten ist bekannt, dass die Spannung in den Metallschichten vom Druck in der UHV-Kammer abhängt und dass durch den Einfluss einer Ar-Atmosphäre spannungsfreie Schichten hergestellt werden können [80]. Abbildung 5.14 zeigt die intrinsische Spannung $\sigma_{f}$ in Abhängigkeit vom Ar-Gasdruck für die Materialien Silber und Permalloy [80]. Zu Beginn bei einem Druck von $10^{-7}$ mbar weisen die AgSchichten eine Spannung von 0,3 GPa und die Permalloy-Schichten von 1,8 GPa auf. Mit steigendem Druck kommt es zu einem Umklappen von Druck- zu Zugspannungen, wobei im Nulldurchgang bei etwa 0,1 mbar die Schichten spannungsfrei aufwachsen.

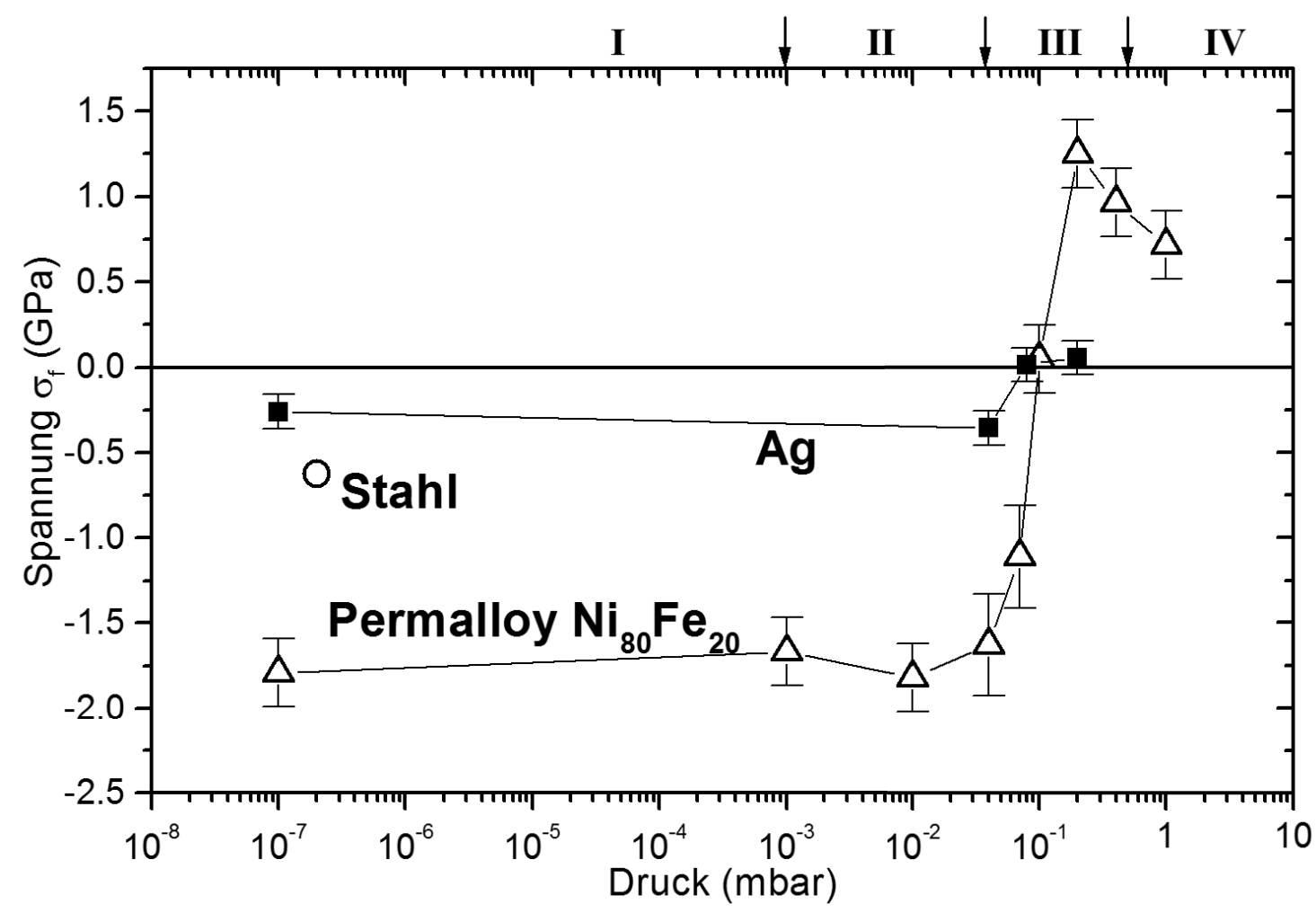

Abbildung 5.14: Abhängigkeit der Spannung vom Ar-Gasdruck in der UHV-Kammer. Negative Werte entsprechen Druckspannungen, positive bedeuten Zugspannungen. Im Nulldurchgang bei 0, 08 bzw. 0,1 mbar wachsen die Schichten spannungsfrei auf (Daten aus [80]).

Dies bietet eine weitere Möglichkeit den Zusammenhang zwischen den Spannungen in der $\mathrm{Cu}$-Schicht und der resultierenden Wellenlänge zu untersuchen. Daher wurden $1 \mu \mathrm{m}$ dicke PMMA-Schichten bei normalen Herstellungsbedingungen, mit einem Druck 
in der UHV-Kammer von $p=10^{-6}$ mbar, deponiert und anschließend mit $60 \mathrm{~nm} \mathrm{Cu}$ bei variierendem Gasdruck beschichtet. Der Druck in der Kammer wurde durch das Einlassen von Ar-Gas variiert. In Abbildung 5.15 sind beispielhaft vier AFM-Aufnahmen von PMMA/Cu-Schichten gezeigt, welche bei unterschiedlichen Drücken von 0,002 mbar, 0,07 mbar, 0,1 mbar und 0,2 mbar hergestellt wurden. Alle Aufnahmen zeigen Wellen, wobei die Wellenlänge bis zu einem Druck von ca. 0,1 mbar kontinuierlich abnimmt und anschließend ab einem Druck von 0,2 mbar wieder stark ansteigt.
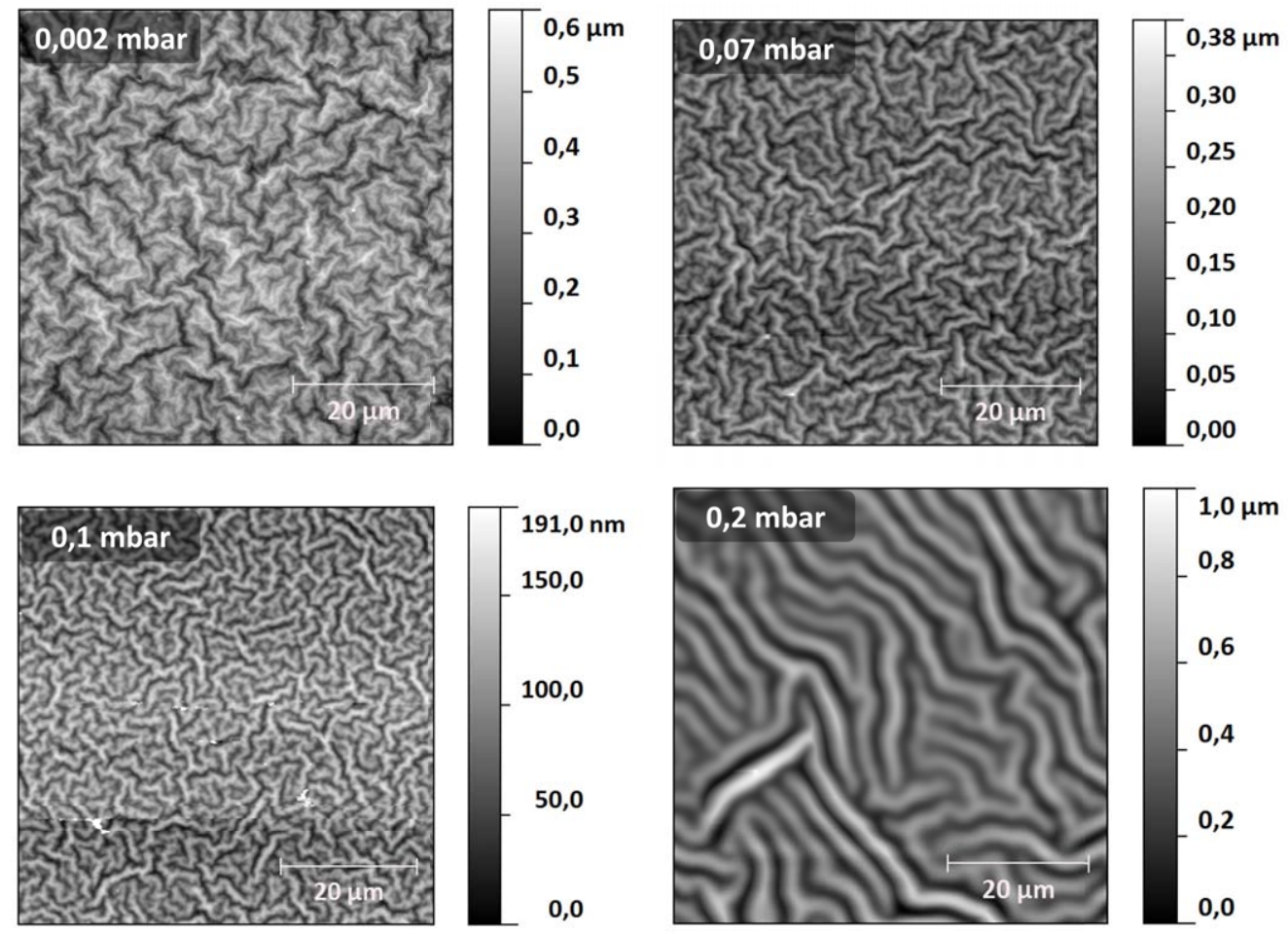

Abbildung 5.15: AFM-Aufnahmen von PMMA/Cu-Schichten, welche bei unterschiedlichen Gasdrücken in der Herstellungskammer deponiert wurden: 0, 002 mbar, 0, 07 mbar, 0, 1 mbar, 0, 2 mbar. Mit steigendem Gasdruck nimmt die Wellenlänge im PMMA/Cu-System ab, bis sie bei Gasdrücken von $0,1-0,2$ mbar wieder ansteigt.

Betrachtet man die dazugehörige RMS-Rauigkeit, welche in der Graphik 5.16 gegenüber dem Druck aufgetragen ist, so zeigt sich folgender Kurvenverlauf. Zunächst nimmt die RMS-Rauigkeit von ca. $100 \mathrm{~nm}$ nur langsam auf etwa $60 \mathrm{~nm}$ ab, bis zu einem Druck von 0,03 mbar. In diesem Bereich, welcher von Sturm als Bereich II bezeichnet wurde (siehe Abbildung 5.14), existiert noch die bekannte Wellenstruktur, welche sich auch bei den normalen Herstellungsdrücken um die $10^{-8}$ mbar ausgebildet hat. Somit sollten im Bereich um die $10^{-3}$ mbar also noch die gleichen inneren Spannungen vorliegen, wie unter üblichen Herstellungsbedingungen. Bis zu einem Druck von 
0, 1 mbar nimmt die RMS-Rauigkeit, von anfangs $89 \mathrm{~nm}$ auf $29 \mathrm{~nm}$ stark ab, wie auch die AFM-Aufnahmen bereits bezüglich der Wellenlänge in Abb. 5.15 gezeigt haben. In diesem Druckbereich III kommt es nun zu einem vermehrten Zusammenstoß der ArIonen mit den $\mathrm{Cu}$-Ionen, wobei diese ihre kinetische Energie weitestgehend verlieren und ungerichtet auf das Substrat treffen. In diesem Bereich findet nun der Übergang von Druckspannungen zu einer spannungsfreien Metallschicht statt. Es zeigt sich, dass dieser Übergangsbereich sehr schmal ist und es somit äußerst schwierig ist, den spannungsfreien Zustand exakt einzustellen. Erhöht man den Druck, so kommt es zum Übergang zu Zugspannungen, was sich neben der starken Zunahme der RMS-Rauigkeit auf bis zu $300 \mathrm{~nm}$ bei 0,4 mbar auch in der Ausbildung großer Wellen in den AFMAufnahmen zeigt. Die Zugspannungen entstehen aufgrund der niedrigen kinetischen Energie der deponierten Teilchen, welche nun die Schicht nicht mehr verdichten, was zu einer porösen Struktur der Schichten führt.

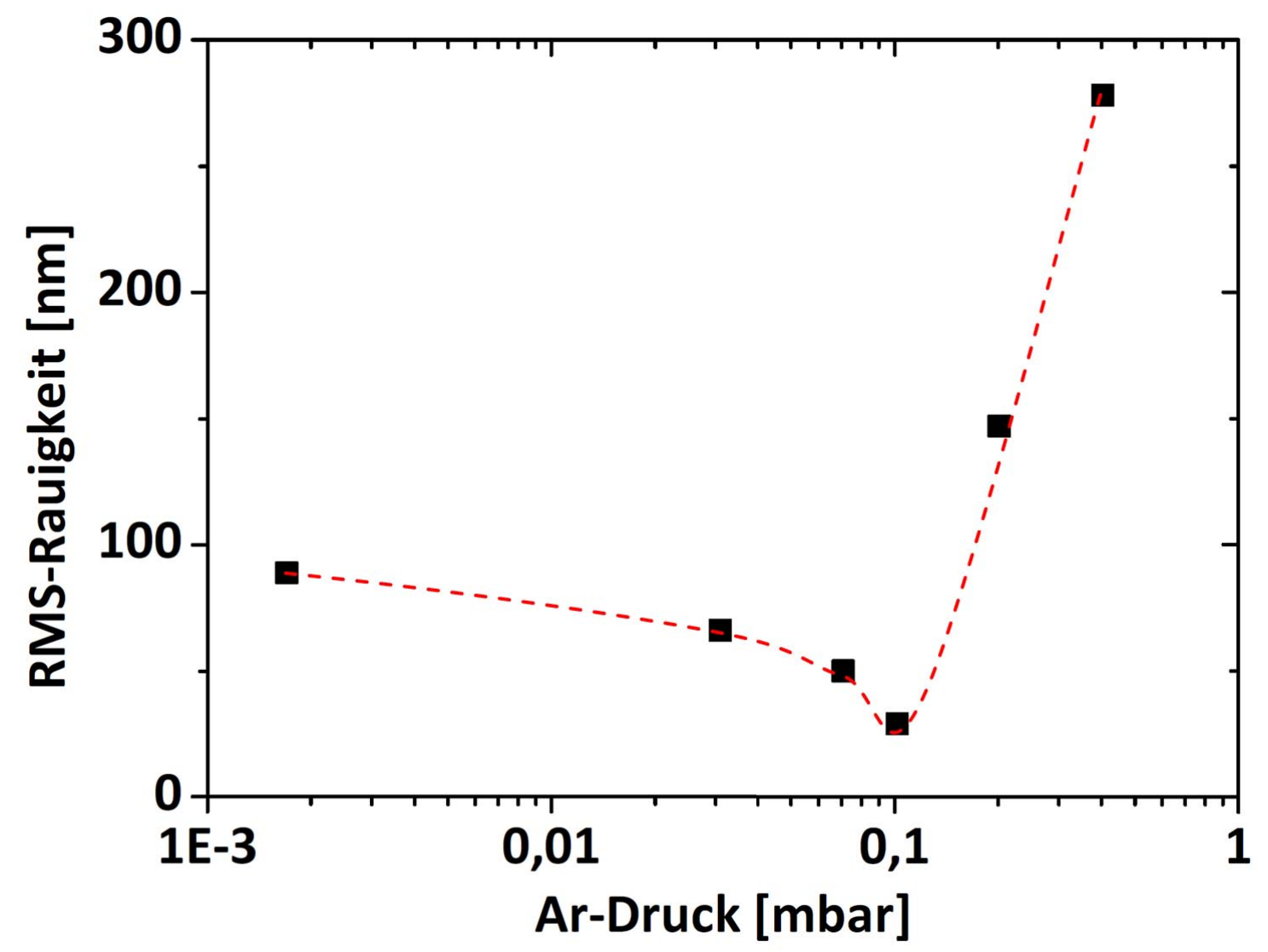

Abbildung 5.16: Abhängigkeit der RMS-Rauigkeit von PMMA/Cu-Schichten, welche bei unterschiedlichen Ar-Gasdrücken in der Herstellungskammer deponiert wurden.

Insgesamt konnte gezeigt werden, dass die Wellenlänge auch abhängig vom Herstellungsdruck in der Kammer ist. Dies zeigt, dass je nach Spannungszustand, welcher 
durch den Druck eingestellt werden kann, die Wellenlänge ganz gezielt variiert werden kann. Insbesondere in dem Druckbereich um 0,1 mbar ist es möglich spannungsfreie $\mathrm{Cu}$-Schichten herzustellen, welche dann glatt auf den PMMA-Schichten aufwachsen. Allerdings ist dieser Druckbereich sehr schmal und somit experimentell schwierig einzustellen. Desweiteren hat sich gezeigt, dass sich die Wellen auch unter Zugspannung ausbilden.

\subsection{Ausblick und Anwendungen}

Mit dem gewonnenen Verständnis über die Entstehung und Beeinflussung der Wellen, soll nun in diesem Kapitel gezeigt werden, welches Anwendungspotenzial die Wellen im Polymer/Metall-System bieten. Insbesondere durch Verwendung der SIEBIMMMethode ist es möglich den Elastizitätsmodul für verschiedenste laserdeponierte Schichten zu bestimmen. Damit hat sich eine neue Messmethode für die Bestimmung von Elastitzitätsmoduln etabliert. Desweiteren soll gezeigt werden, dass sich die Wellen gezielt anordnen lassen, was eine Vielzahl von Wellen-Designs ermöglicht.

\subsubsection{SIEBIMM-Methode}

In Kapitel 5.2 konnte der Elastizitätsmodul für die PMMA-Schichten mit $E_{P}=3 \mathrm{MPa}$ bestimmt werden. Dadurch sollte es nun möglich sein bei bekannter Wellenlänge und Schichtdicke den E-Modul $E_{M}$ für verschiedenste dünne, laserdeponierte Schichten mit folgender Gleichung zu bestimmen.

$$
E_{M}=3 E_{P}\left(\frac{\lambda}{2 \pi h_{M}}\right)^{3}
$$

Die Verwendung der Formel zeigt nur Gültigkeit, wenn man einen Substrateinfluss ausschließen kann, auf den bereits in Kapitel 5.2 eingegangen wurde. Daher sind bei der Schichtherstellung folgende Bedingungen wesentlich. Zum einen muss die PMMASchicht ausreichend dick sein. Hier hat sich bereits gezeigt, dass bei Verwendung eines PMMA-Substrates, die deponierte PMMA-Schicht mindestens eine Schichtdicke von $6 \mu \mathrm{m}$ aufweisen muss, um den Substrateinfluss zu eliminieren. Desweiteren darf die deponierte zu untersuchende Schicht nicht zu dick sein, da mit steigender Schichtdicke die Wellenlänge und Amplitude der Wellen zunimmt und somit bei zu starker 
Deformation der Schicht das Substrat wieder einen Einfluss hat. Allerdings darf die zu untersuchende Schicht auch nicht zu dünn gewählt werden, da insgesamt eine geschlossene Schicht benötigt wird.
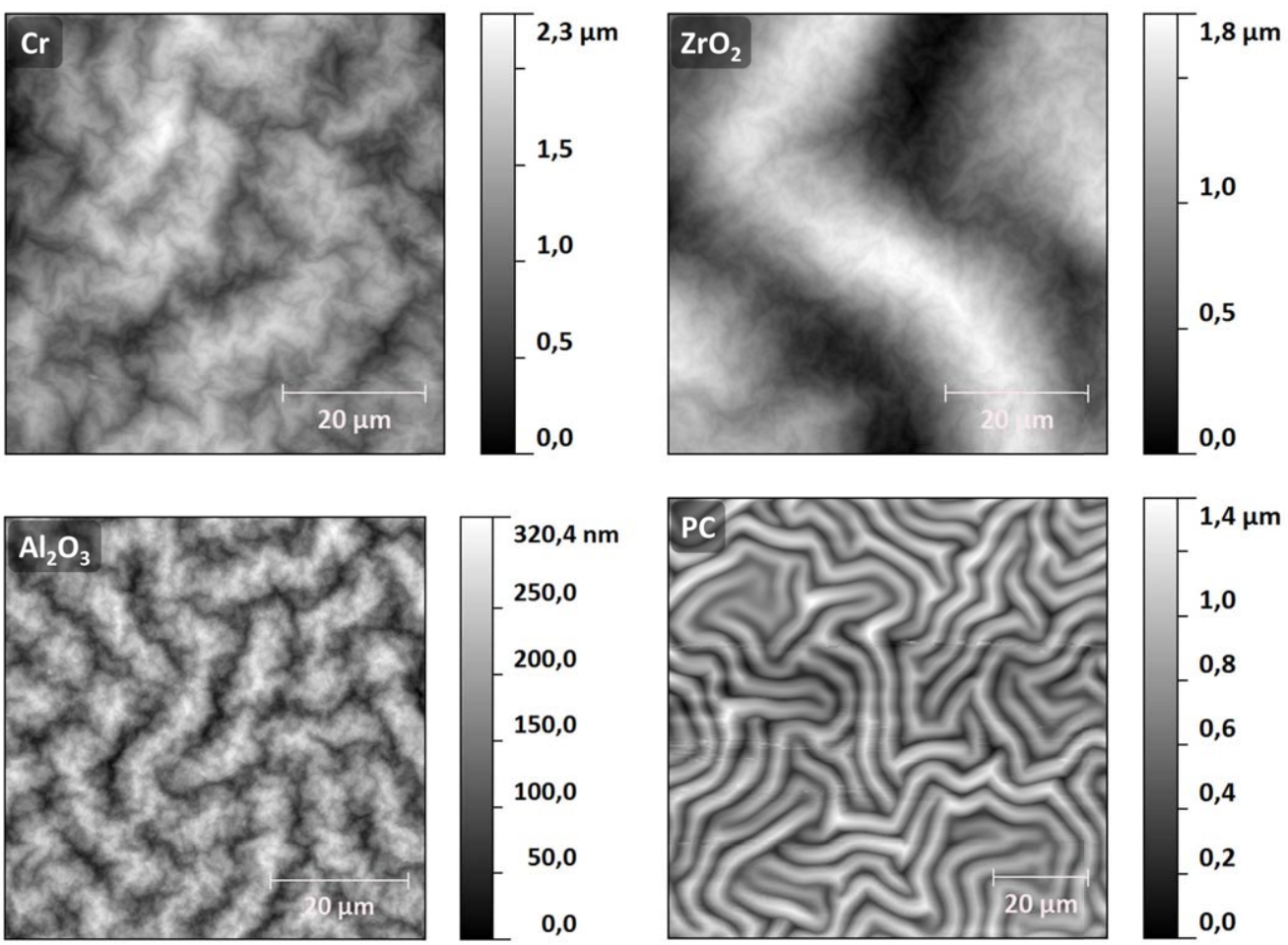

Abbildung 5.17: AFM-Aufnahmen von $50 \mathrm{~nm}$ dicken $\mathrm{Cr}-, \mathrm{ZrO}_{2}-, \mathrm{Al}_{2} \mathrm{O}_{3^{-}}$und PC-Schichten. Hierbei wurden die $\mathrm{Cr}$ - und $\mathrm{ZrO}_{2}$-Schichten auf 6,3 $\mu$ m dicken PMMA-Schichten deponiert, welche sich auf einem PMMA-Target befanden. Die $\mathrm{Al}_{2} \mathrm{O}_{3}$-Schicht wurde auf einer $450 \mathrm{~nm}$ dicken PMMA-Schicht deponiert und die PC-Schicht auf einer $1 \mu \mathrm{m}$ dicken PMMA-Schicht, beide Schichtsysteme wurden auf Si-Substraten deponiert. Alle Materialien zeigen Wellenbildung. Dabei tritt im PMMA/PCSystem lediglich eine Generation an Wellen auf, wohingegen alle anderen Materialien Doppelwellen aufweisen.

In Abbildung 5.17 sind AFM-Aufnahmen von $50 \mathrm{~nm}$ dicken $\mathrm{Cr}-, \mathrm{ZrO}_{2^{-}}, \mathrm{Al}_{2} \mathrm{O}_{3^{-}}$und PC-Schichten dargestellt. Hierbei wurden die $\mathrm{Cr}$ - und $\mathrm{ZrO}_{2}$-Schichten auf $6,3 \mu \mathrm{m}$ dicken PMMA-Schichten deponiert, welche sich auf einem PMMA-Target befanden. Dahingegen wurde die $\mathrm{Al}_{2} \mathrm{O}_{3}$-Schicht auf einer $450 \mathrm{~nm}$ dicken PMMA-Schicht und die PC-Schicht auf einer $1 \mu \mathrm{m}$ dicken PMMA-Schicht deponiert, beide Schichtsysteme wurden auf Si-Substraten deponiert. Es zeigt sich, dass sowohl die Cr-, die $\mathrm{ZrO}_{2}-$ und die $\mathrm{Al}_{2} \mathrm{O}_{3}$-Schichten Doppelwellen ausbilden, wohingegen die PC-Schicht lediglich Wellen einer Generation aufweist. Insbesondere die Wellenbildung bei der PC-Schicht ist interessant, da hier der Ablationsprozess anders abläuft als bei den Metallen und Oxiden. Wie schon in Kapitel 4.2 gezeigt, kommt es beim Ablationsprozess der 
Polymere zu einem Übertrag von ganzen Polymerketten, welche sich auf dem Substrat niederschlagen [50]. Die Ausbildung der Wellen mit einer Wellenlänge von $\lambda=4 \mu \mathrm{m}$ weist also daraufhin, dass sich auch bei diesem anders ablaufendem Ablationsprozess Spannungen in der Schicht aufbauen. Wie im Detail dieser Prozess abläuft konnte bis jetzt noch nicht geklärt werden. Auf den ersten Blick ist in den AFM-Aufnahmen $\mathrm{zu}$ erkennen, dass im Falle der $\mathrm{ZrO}_{2}$-Schicht sehr große Wellen auftreten mit einer Wellenlänge von $\lambda=25 \mu \mathrm{m}$ (2. Generation). Dahingegen haben die Wellen der CrSchicht eine Wellenlänge von $\lambda=10 \mu \mathrm{m}$ und die Wellen der $\mathrm{Al}_{2} \mathrm{O}_{3}$-Schicht eine Wellenlänge von $\lambda=8,5 \mu \mathrm{m}$.

Mit Kenntnis der Wellenlänge kann nun die obige Formel angewendet werden, um den Elastizitätsmodul der Schichten zu bestimmen. Die Ergebnisse sind in der folgenden Tabelle 5.2 dargestellt. Zum Vergleich wurde an reinen laserdeponierten Schichten aus $\mathrm{Cr}, \mathrm{ZrO}_{2}, \mathrm{Al}_{2} \mathrm{O}_{3}$ und PC mittels Nanoindentierung der E-Modul gemessen [74]. Es zeigt sich, dass der über die SIEBIMM-Methode berechnete E-Modul für die CrSchicht mit einer Abweichung von 15\% nur gering von den Nanoindentermessungen abweicht. Im Gegensatz dazu, weicht der berechnete E-Modul für die $\mathrm{ZrO}_{2}$-Schicht um mehr als $2000 \%$ von den Nanoindentermessungen ab. Wie aus den AFM-Aufnahmen ersichtlich, bildet $\mathrm{ZrO}_{2}$ bei einer Schichtdicke von $50 \mathrm{~nm}$ schon sehr große Wellen mit einer Wellenlänge von $25 \mu \mathrm{m}$ aus. Dies weist auf eine starke Deformation der $\mathrm{ZrO}_{2}$ Schicht hin, wodurch möglicherweise erneut der Einfluss des Substrates für die große Abweichung des E-Moduls verantwortlich gemacht werden kann. Auch der E-Modul für die $\mathrm{Al}_{2} \mathrm{O}_{3}$-Schicht weicht nur sehr gering mit $12 \%$ von der Nanoindentermessung ab, und das obwohl hier zum einen die dünnste PMMA-Schicht mit nur $450 \mathrm{~nm}$ und ein SiSubstrat verwendet wurde. Eine mögliche Erklärung für das gute Messergebnis könnte in der ausgebildeten Amplitude der Wellen liegen, welche gerade mal $170 \mathrm{~nm}$ beträgt. Im Vergleich dazu weisen alle anderen Schichten wesentlich größere Amplituden auf,

\begin{tabular}{|l|c|c|c|}
\hline Material & $h_{M}[\mathrm{~nm}]$ & $E_{\text {SIEBIMM }}[\mathrm{GPa}]$ & $E_{\text {Nanoindenter }}[\mathrm{GPa}]$ \\
\hline $\mathrm{Cr}$ & 50 & 290 & 253 \\
\hline $\mathrm{ZrO}_{2}$ & 50 & 4535 & 203 \\
\hline $\mathrm{Al}_{2} \mathrm{O}_{3}$ & 50 & 178 & 200 \\
\hline $\mathrm{PC}$ & 50 & 18 & 6 \\
\hline
\end{tabular}

Tabelle 5.2: Vergleich der über die SIEBIMM-Methode berechneten Elastizitätsmodule mit E-ModulWerten aus Nanoindentermessungen für Cr-, $\mathrm{ZrO}_{2-}, \mathrm{Al}_{2} \mathrm{O}_{3^{-}}$und PC-Schichten. 
wie sich auch durch einen einfachen Blick auf die Höhenskalen der AFM-Aufnahmen zeigt. Somit liegt möglicherweise bei den $\mathrm{Al}_{2} \mathrm{O}_{3}$-Schichten nur ein sehr geringer Substrateinfluss durch das verwendete Si-Substrat vor. Die PC-Schichten weisen wiederum eine sehr große Abweichung mit $67 \%$ von den Nanoindentermessungen auf, was auf einen großen Substrateinfluss schließen lässt. Dies könnte aber auch auf einen anders ablaufenden Ablationsprozess, wie oben schon erwähnt, zurückgeführt werden. Für zukünftige Messungen ist es wichtig, die deponierte Schicht so dünn wie möglich zu wählen, um den Substrateinfluss zu verhindern. Am Beispiel $\mathrm{Cr}$ und $\mathrm{Al}_{2} \mathrm{O}_{3}$ zeigt sich, dass die neue Messmethode ein großes Potential hat.

Zusammenfassend konnte gezeigt werden, dass die neue Messmethode auch für die Bestimmung von Elastizitätsmoduln verschiedenster laserdeponierter Schichten verwendet werden kann. Allerdings ist dabei die Vermeidung des Substrateinflusses von großer Bedeutung für ein genaues Ergebnis. Desweiteren hat sich gezeigt, dass auch Oxide und Polymere Wellenbildung zeigen, welches die Messmethode in ihrer Vielfältigkeit noch erweitert.

\subsubsection{Anordnen der Wellen}

Im Allgemeinen ist die Ausrichtung der Wellen auf der Metalloberfläche statistisch verteilt, da beim Herstellen der Schicht ein isotropes Spannungsfeld erzeugt wird. Allerdings zeigt sich, dass die Wellen in ihrer Ausrichtung schon durch kleine Unregelmäßigkeiten, wie zum Beispiel einem Droplet, beeinflusst werden. Abbildung 5.18 zeigt eine REM-Aufnahme einer $1 \mu \mathrm{m}$ dicken PMMA-Schicht mit $50 \mathrm{~nm}$ deponiertem $\mathrm{Cu}$. Im Zuge der Laserdeposition beider Materialien treten als Artefakte auch vereinzelt Droplets auf, welche die Wellenbildung nun derart beeinflussen, dass die Wellen strahlenförmig von diesen weglaufen. Dabei beträgt die Wellenlänge der teilweise angeordneten Wellen $1 \mu \mathrm{m}$. Desweiteren weist der große Droplet in der REM-Aufnahme eine teilweise Anordnung der Wellen in einem Radius von $10 \mu \mathrm{m}$ um sich herum auf.

Um die Wellen nun gezielt anzuordnen, wurde die PMMA-Schicht nach der Herstellung mittels Elektronenstrahllithographie (in Zusammenarbeit mit Christin Kalkert) strukturiert. Die Bestrahlung des Polymers mit Elektronen sollte hier eine punktuelle Härtung des Materials bewirken [81], wodurch die Wellenbildung beeinflusst werden könnte. In Bezug auf die runden Droplets wurden Strukturen in Form von Kreisen und Stegen bestrahlt. Anschließend wurden $50 \mathrm{~nm} \mathrm{Cu}$ auf die strukturierte PMMA-Schicht deponiert. 


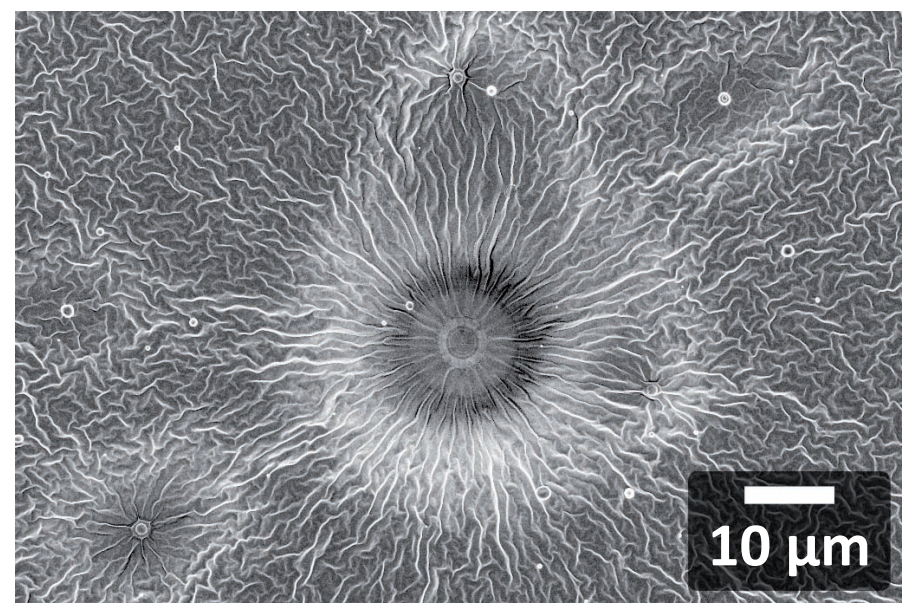

Abbildung 5.18: REM-Aufnahme einer $1 \mu \mathrm{m}$ dicken PMMA-Schicht mit $50 \mathrm{~nm} \mathrm{Cu}$. Es zeigt sich, dass die Ausrichtung der Wellen von kleinen Unregelmäßigkeiten, wie z.B. Droplets, beeinflusst werden kann.
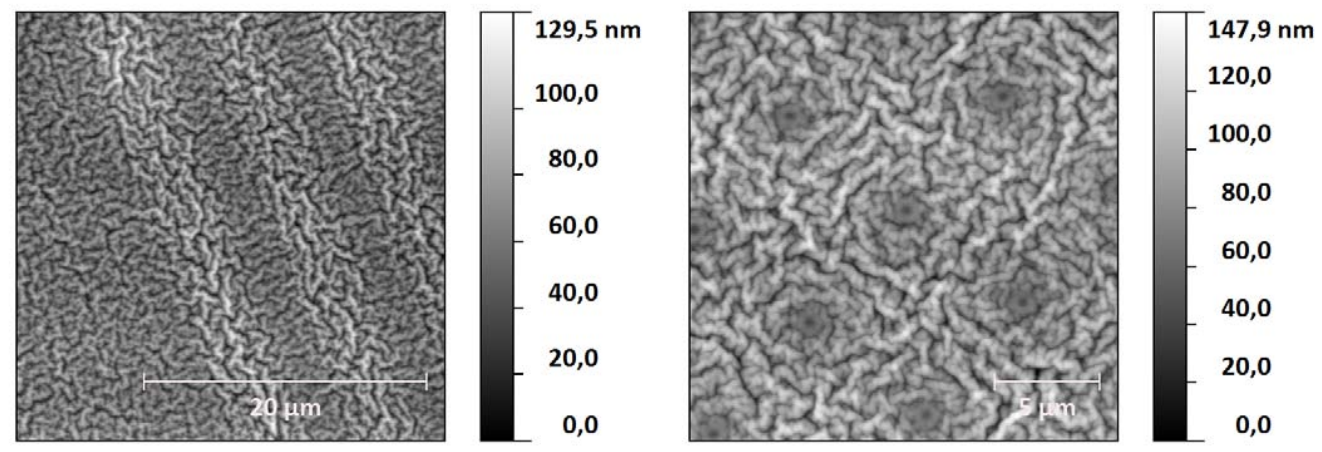

Abbildung 5.19: AFM-Aufnahmen zweier Proben, bei denen die PMMA-Schicht nach der Herstellung und vor der $\mathrm{Cu}$-Beschichtung mit Elektronenstrahllithographie behandelt wurde. Hierbei wurden Stege (links) und Punktmuster (rechts) auf die PMMA-Probe gestrahlt. Anschließend sind $50 \mathrm{~nm}$ $\mathrm{Cu}$ deponiert worden. Es zeigt sich kaum ein Einfluss auf die Ausrichtung der Wellen.

In Abbildung 5.19 sind AFM-Aufnahmen der strukturierten Probe gezeigt. Auf den ersten Blick lässt sich jedoch erkennen, dass die Wellen in ihrer Ausrichtung kaum beeinflusst wurden. Die Stege, welche im AFM-Bild links hell erscheinen, haben keinen Einfluss auf die Ausrichtung der Wellen bewirkt. Bei dem Punktmuster ist eine leichte Ausrichtung zwischen zwei Punkten zu erkennen. Der Grund für die geringe Beeinflussung der Wellen ist in den zu geringen Dosen des Elektronenstrahls zu vermuten. Die PMMA-Schicht hat sich durch die Bestrahlung mit dem Elektronenstrahl anscheinend nicht ausreichend verändert, so dass sich die Wellen ansatzweise normal ausbilden konnten. 
Eine andere Herangehensweise die Ausrichtung der Wellen zu beeinflussen, besteht darin, ein richtungsabhängiges Spannungsfeld zu erzeugen. Hierfür wurde die Deposition von $\mathrm{Cu}$ unter einem Winkel von 23 durchgeführt. Da die Depositionsrate quadratisch vom Target-Substrat-Abstand abhängt, konnte so auf der Probe ein Schichtdickengradient und somit ein anisotropes Spannungsfeld erzeugt werden, da die Spannung von der Schichtdicke abhängt. Die Deposition einer 500 nm dicken PMMASchicht geschah unter normalen Bedingungen (senkrechte Deposition). Anschließend wurde die Probe auf einen Keil aufgeklebt, so dass die Deposition von $\mathrm{Cu}$ unter besagtem Winkel stattfand. Anschließend sind 51000 Pulse auf das $\mathrm{Cu}$-Target abgegeben worden. Der experimentelle Aufbau ist in Abbildung 5.20 veranschaulicht.

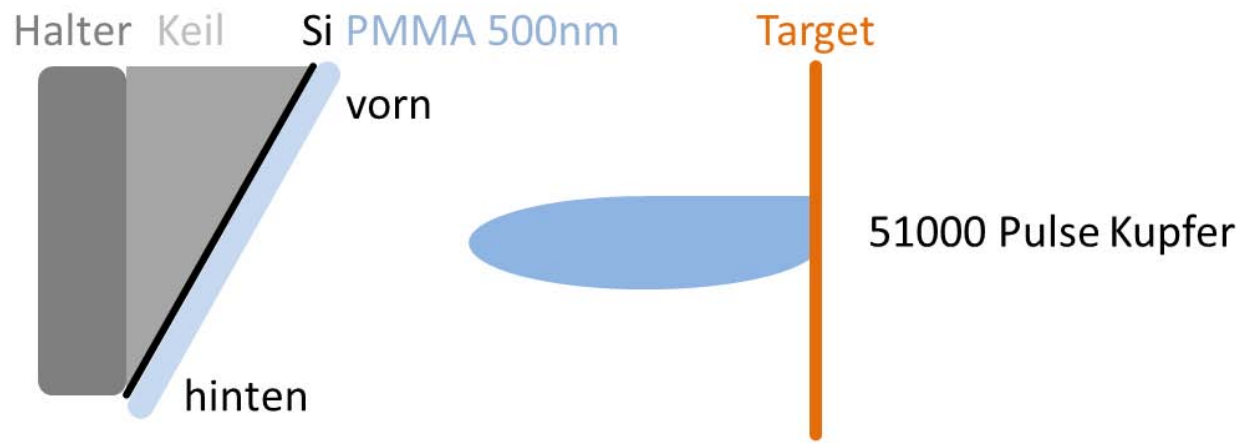

Abbildung 5.20: Schematische Darstellung der Probenherstellung zur Erzeugung eines Schichtdickengradients der $\mathrm{Cu}$-Schicht auf einer $500 \mathrm{~nm}$ dicken PMMA-Schicht durch die Verwendung eines Keils.

Die REM-Aufnahmen in Abbildung 5.21 zeigen, dass sich die Wellen auf der Probe ganz unterschiedlich ausgebildet haben und zwar abhängig von der Position auf dem Substrat. Wie in der schematischen Darstellung (s. Abb. 5.20) zu sehen, wird mit der Bezeichung „vorn“ die Position auf dem Substrat bezeichnet, welche den kleinsten Target-Substrat-Abstand aufweist. Die Wellen haben sich an dieser Stelle statistisch verteilt ausgerichtet, wie es bereits aus vorangegangenen Messungen bekannt ist. In der Mitte der Probe ist allerdings zu beobachten, dass sich die Wellen quer zur Längsseite des Substrates teilweise ausgerichtet haben. Betrachtet man den „hinteren“ Teil des Substrates, welcher den größten Target-Substrat-Abstand aufweist, so zeigen die Wellen hier ein ganz neues Aussehen. Zwar lässt sich eine leichte Ausrichtung erahnen, aber nicht mehr über eine so lange Distanz, wie in der Probenmitte.

Dieses Verhalten lässt sich wie folgt interpretieren. Als erstes ist anzunehmen, dass die $\mathrm{Cu}-\mathrm{Schicht}$ vorne am Substrat insgesamt die dickste Schicht auf der Probe ist, wohingegen die $\mathrm{Cu}$-Schichtdicke hinten am Substrat am dünnsten ist. Somit weist die Probe einen Schichtdickengradienten entlang der Längsseite des Substrates auf. Da 

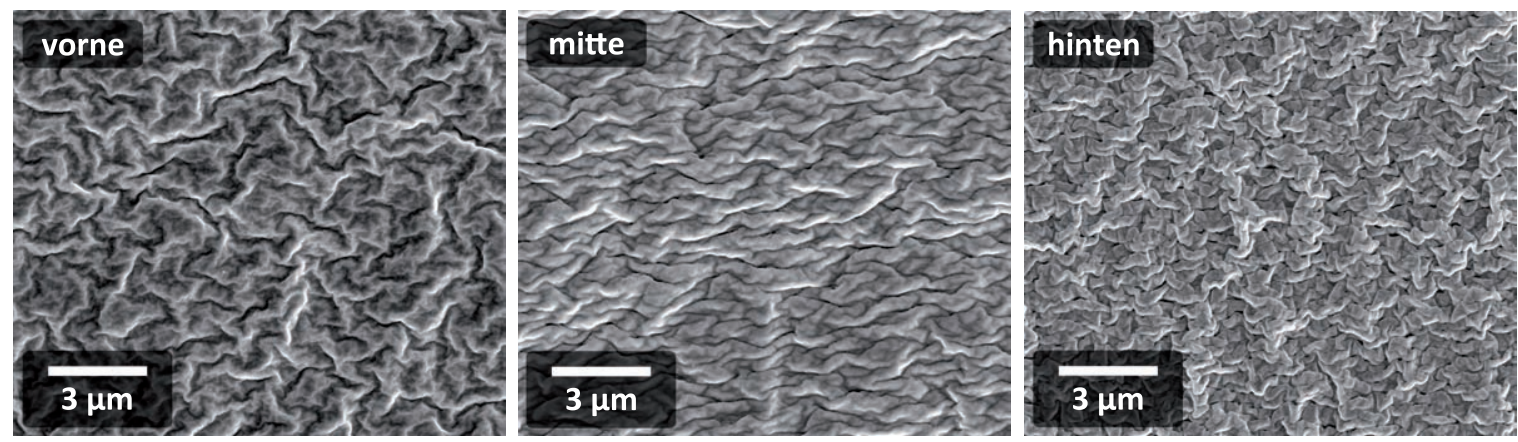

Abbildung 5.21: REM-Aufnahmen von drei unterschiedlichen Positionen der Probe aus dem KeilExperiment. In der Mitte der Probe ist eine teilweise Ausrichtung der Wellen quer zur Längsseite des Substrates zu erkennen.

aber nun die Spannungen in den Cu-Schichten von der Schichtdicke abhängen, wie im vorherigen Kapitel gezeigt werden konnte, kommt es dadurch auch zu einem Spannungsgradienten in der Probe, der die Probe von der Mitte ausgehend auseinanderzieht und somit die Ausrichtung der Wellen beeinflusst. Dieser Prozess geschieht selbstorganisiert, und die Wellen richten sich teilweise über die komplette Breitseite des Substrates $(1 \mathrm{~cm})$ aus. Im Folgenden wurden die angeordneten Wellen des mittigen Probenbereichs gezielt untersucht. Die AFM-Aufnahme der Probenmitte in Abbildung 5.22 zeigt, dass sich Doppelwellen ausgebildet haben, bei denen sich jedoch lediglich die großen Wellen der 2. Generation ausgerichtet haben. Dabei ist die Ausrichtung der Wellen nicht perfekt, sondern nur teilweise zu beobachten. Die REM-Aufnahme in Abbildung 5.22 verdeutlich zusätzlich noch einmal die Reichweite der teilweise ausgerichteten Wellen über eine Strecke von bis zu $150 \mu \mathrm{m}$.
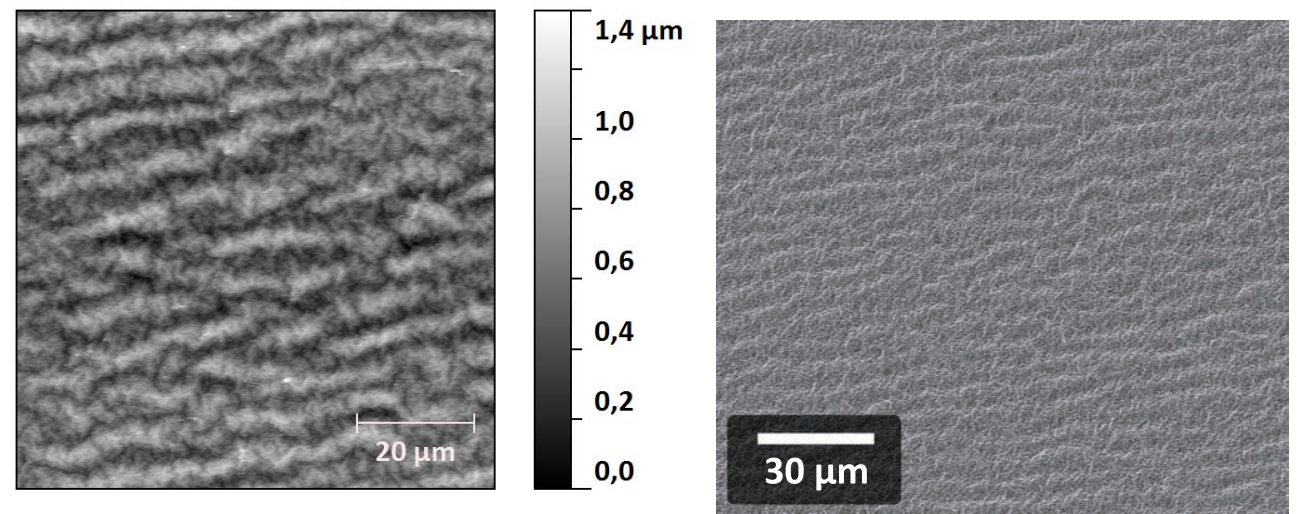

Abbildung 5.22: AFM-Aufnahme (linkes Bild) und REM-Aufnahme (rechtes Bild) von der Mitte der Probe aus dem Keil-Experiment. 
Ausgehend von diesen ersten Experimenten zur Anordnung von Wellen im Polymer/Metall-System wurde in Zusammenarbeit mit Anja Westphal [82] im System Pd/PMMA die Anordnung der Wellen durch verschiedene Verfahren wie Strukturierung des PMMA, Ritzen von Linien in die PMMA-Schicht, Abtrag von Teilen der PMMASchicht zur Erzeugung von Stegen und Deposition der Metallschicht durch eine Blende systematisch weiter untersucht. Es zeigte sich, dass insbesondere durch ein Anritzen der PMMA-Schicht scharfkantige Gräben durch die PMMA-Schicht bis auf das Substrat erzeugt werden konnten. Nach der Deposition von nur $7 \mathrm{~nm}$ Pd auf die angeritzte Polymerschicht (Schichtdicke 3,3 $3 \mathrm{~m}$ ) wurde eine Ausrichtung der Wellen auf der Metallschicht senkrecht zu den geritzten Linien erzielt. Diese Anordnung der Wellen senkrecht $\mathrm{zu}$ den geritzten Linien ist in Abbildung 5.23 gut zu erkennen. Im linken Teilbild wird zudem ersichtlich, dass die Anordnung der Wellen, sobald keine parallelen Linien mehr vorliegen, wieder in eine statistische Verteilung übergeht. Diese senkrechte Anordnung der Wellen, ausgehend von den Linien, führt bei gekreuzten RitzLinien zu dem spannenden Effekt, dass sich an den Kreuzungspunkten ringförmige Wellenstrukturen ausbilden. Im Probenbereich weit entfernt von den Linien oder Kreuzungspunkten (siehe Bildmitte) liegt wie zu erwarten eher wieder eine statistische Verteilung der Wellen vor. Insgesamt ist eine Ausrichtung der Wellen über eine Distanz von $500 \mu \mathrm{m}$ (Abstand der Kanten) zu beobachten.
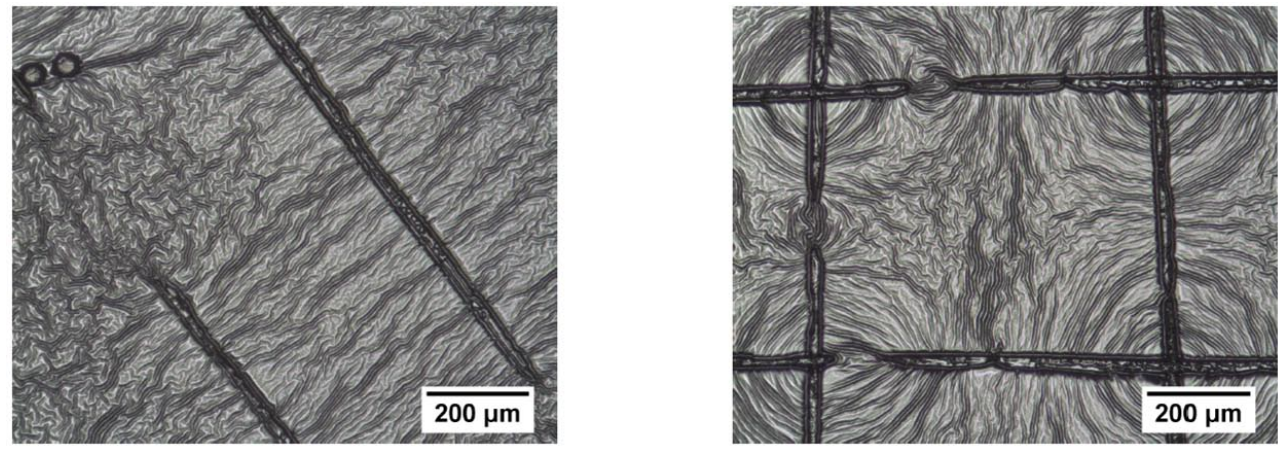

Abbildung 5.23: Lichtmikroskopische Aufnahmen von Wellen einer PMMA/Pd-Schicht, die sich an Linien, die in die PMMA-Schicht geritzt wurden, senkrecht anordnen. Die Pd-Schicht ist $7 \mathrm{~nm}$ und die PMMA-Schicht 3, $3 \mu \mathrm{m}$ dick (Daten aus [82]).

In der Literatur findet man ein breites Spektrum von verschiedenen Varianten zur Erzeugung und Anordnung von Wellen. Hierbei haben sich insgesamt zwei Prozesse zum Anordnen der Wellen etabliert. Dies ist zum einen die Anordnung der Wellen durch Strukturierung des Substrates und zum anderen die Ausrichtung der Wellen in Richtung einer angelegten Spannung. In der Gruppe um Bowden et al. werden 
Wellen erzeugt, indem eine Metallschicht (Ti, Au) auf ein PDMS-Substrat bei erhöhter Substrattemperatur deponiert wird, und anschließend die Probe wieder auf Raumtemperatur abgekühlt wird. Durch das Zusammenziehen der Schichten beim Abkühlen entstehen Spannungen, welche zur Ausbildung der Wellen mit einer Wellenlänge von $30 \mu \mathrm{m}$ führen [10,11]. Durch das Strukturieren des PDMS-Substrates lassen sich die Wellen an eckigen und kreisförmigen Strukturen anordnen, wie in Abbildung $5.24 \mathrm{zu}$ erkennen ist.
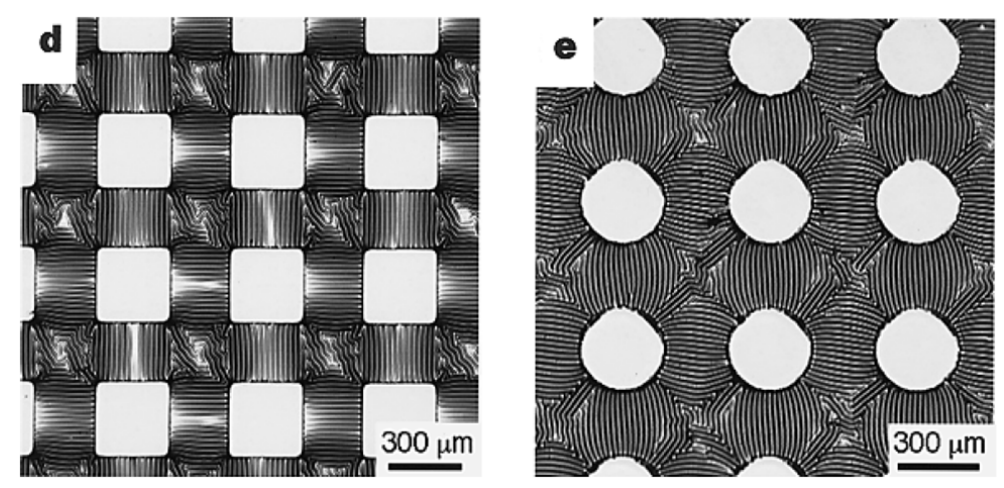

Abbildung 5.24: Lichtmikroskopische Aufnahmen eines strukturierten PDMS-Subtrats, dass mit $5 \mathrm{~nm}$ Titan und $50 \mathrm{~nm}$ Gold beschichtet wurde. Die Wellen ordnen sich sowohl an eckigen als auch kreisförmigen Strukturen an (Daten aus [10]).

Die Gruppe um Chua et al. erzeugt Wellen auf einem PDMS-Substrat, indem es die Oberfläche durch eine Plasmabehandlung oxidiert [83]. Eine weitere Methode um Wellen anzuordnen ist auch durch Laserbestrahlung möglich [84]. Hierbei ordnen sich die Wellen in laserbestrahlten Bereichen an. Ferner erzeugt die Gruppe um Stafford et al. Wellen mit Hilfe einer Zugapparatur, welche Spannung auf die Schichten ausübt [16]. Dabei konnten sie zeigen, dass sich die Wellen, je nach Richtung der angreifenden Spannung, beliebig anordnen lassen. Abbildung 5.25 zeigt hier die verschiedenen Wellenmuster, die über diese Methode erzeugt werden konnten. Die Gruppe um Ohzono et al. zeigt, dass das Anordnen der Wellen in Richtung der angreifenden Spannung ein reversibler Prozess ist. Die Wellen begeben sich wieder in ihre statistisch verteilte Ursprungsform, wenn die Spannung reduziert wird [14].

Zusammenfassend konnte gezeigt werden, dass das Anordnen der Wellen durch verschiedene Verfahren möglich ist. Hierbei war das Anordnen durch ein Strukturieren der PMMA-Schicht mittels Elektronenstrahllithographie weniger erfolgreich als es in der Literatur dargestellt wird. Dies lässt sich dadurch erklären, dass die verwendeten Prozesse zum Anordnen der Wellen in der Literatur oftmals durch einen globalen Effekt erzielt wurden, wie z. B. durch eine Zugapparatur, welche den Spannungszustand 


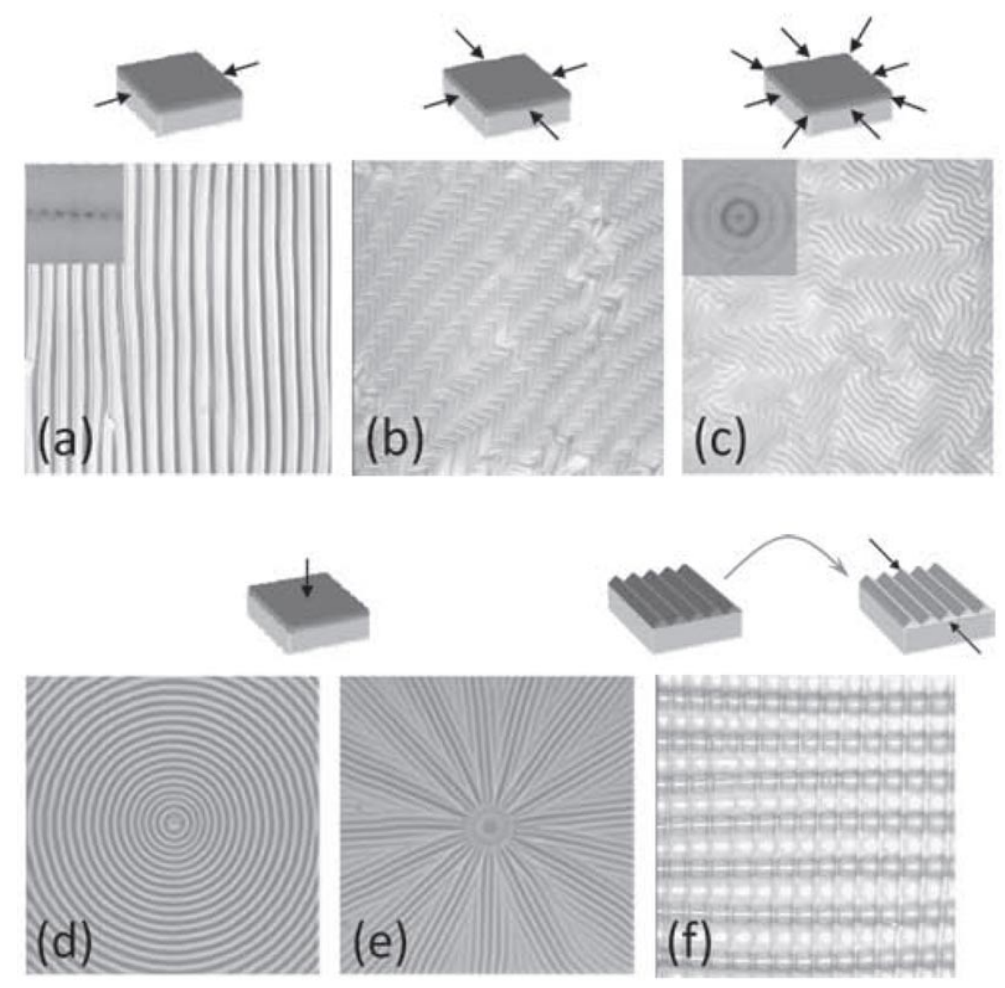

Abbildung 5.25: Lichtmikroskopische Aufnahmen verschiedener Wellenmuster. Je nach Richtung der anliegenden Spannung, ordnen sich die Wellen senkrecht dazu an. Somit ergeben sich verschiedene Strukturen: (a) Streifen (einachsiger Spannungszustand), (b) Fischgrätenmuster (zweiachsiger Spannungszustand), (c) Labyrinth (isotropischer Spannungszustand), (d,e) Zielscheibe/Radspeiche (Lösungsmittelschwellen an einem lokalen Defekt), (f) Schachbrettmuster (einachsiger Spannungszustand auf einem vorstrukturiertem Substrat) (Daten aus [16,85,86]).

über die komplette Probe beeinflussen kann. Die hier verwendeten Verfahren zum Anordnen der Wellen geschahen dabei immer selbstorganisiert, was auf eine lokale Veränderungen der Schicht zurückgeführt werden konnte. Ein Anordnen der Wellen im System PMMA/Pd konnte durch einfaches Ritzen der PMMA-Schicht über eine Reichweite von bis zu $500 \mu \mathrm{m}$ erreicht werden. Desweiteren war es möglich durch die Deposition der Cu-Schicht unter einem Winkel einen Spannungsgradienten auf der Probe zu erzeugen, durch den sich die Wellen selbstorganisiert über eine Reichweite von $1 \mathrm{~cm}$ angeordnet haben. Die Wellen waren dabei nicht perfekt, sondern nur teilweise angeordnet. 



\section{Strategien zum Erreichen glatter Polymer/Metall-Schichtpakete}

In diesem Kapitel soll untersucht werden, wie der Elastizitätsmodul der Polymerschicht beeinflusst werden kann, und welche Auswirkungen das auf die Wellenbildung hat. Das Hauptaugenmerk liegt in diesem Abschnitt der Arbeit in der Vermeidung der Wellen und dem Erzeugen von glatten Schichten, welche sich dann für komplexe Polymer/Metall-Schichtsysteme eignen.

\subsection{Beeinflussung des Elastizitätsmoduls der Polymerschicht}

Die Ausbildung von Wellen in einer PMMA/Cu-Schicht ist zum einen auf die Spannungen in den laserdeponierten $\mathrm{Cu}$-Schichten durch Ionenimplantation und zum anderen auf die weiche PMMA-Unterlage zurückzuführen, welche einen sehr niedrigen Elastizitätsmodul (etwa $3 \mathrm{MPa}$ ) besitzt. In den folgenden Abschnitten soll nun auf verschiedene Arten der Elastizitätsmodul der PMMA-Schicht beeinflusst und verändert werden, um so die Ausbildung der Wellen zu verhindern.

\subsubsection{Beeinflussung des Elastizitätsmoduls durch UV-Bestrahlung}

Eine Möglichkeit, die Vernetzung in den PMMA-Schichten anzuregen, ist eine nachträgliche Bestrahlung der Schichten mit ultraviolettem Licht. Hierfür wurden zunächst mehrere $6 \mu \mathrm{m}$-dicke PMMA-Schichten in-situ mit einer UV-Lampe bestrahlt. Bei der UV-Lampe handelte es sich um eine Quecksilber-Xenon-Lampe Lightningcure LC5 der Firma Hamamatsu $(\lambda=365 \mathrm{~nm})$. Es wurden unterschiedliche Bestrahlungszeiten von $30 \mathrm{~min}$ bis $16 \mathrm{~h}$ verwendet. Anschließend wurde der Elastizitätsmodul der bestrahlten 
PMMA-Schichten mit Hilfe eines Indenters vermessen. In Abbildung 6.1 ist die Abhängigkeit des Elastizitätsmoduls von der Bestrahlungszeit aufgetragen.

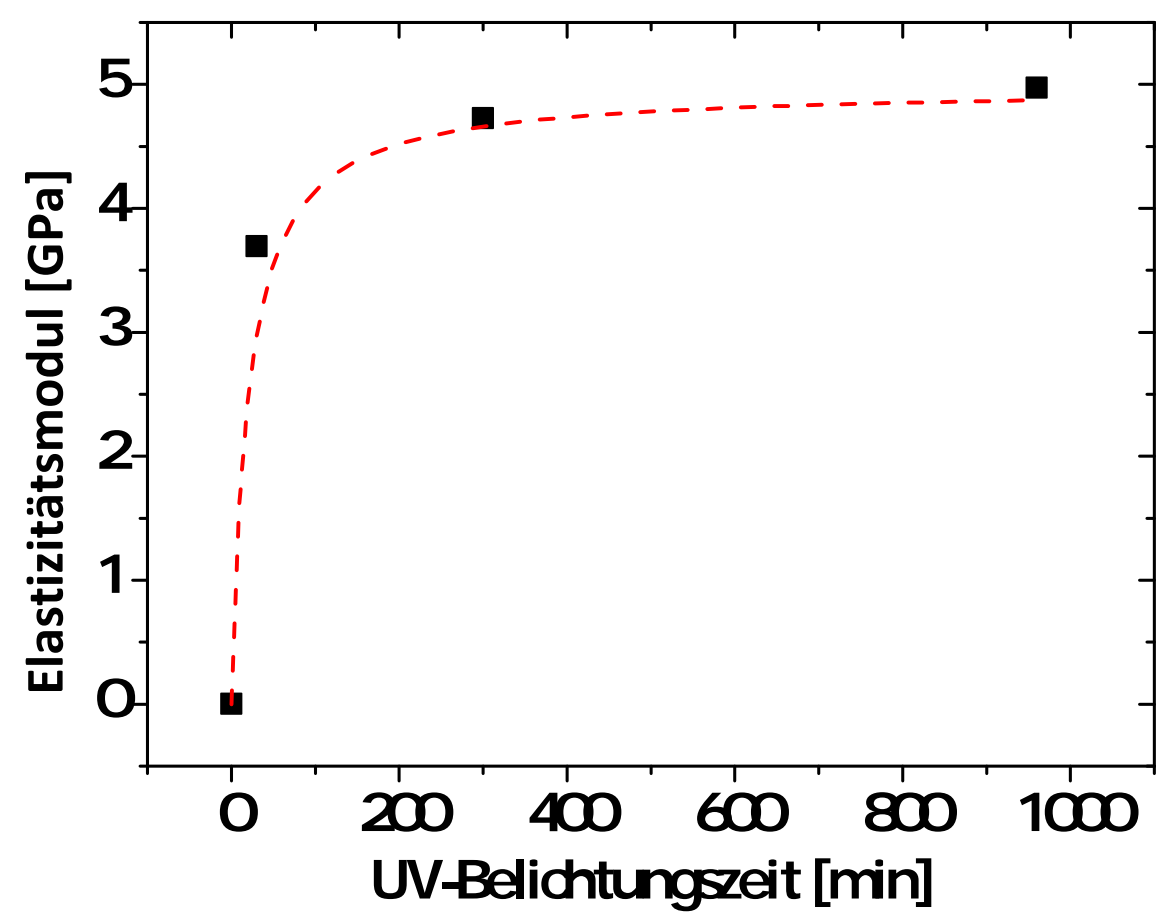

Abbildung 6.1: Elastizitätsmodul in Abhängigkeit der UV-Bestrahlungszeit der PMMA-Schichten. Mit zunehmender Bestrahlungsdauer steigt der E-Modul der PMMA-Schichten, welches aufgrund von angeregter Vernetzung der Ketten zu erklären ist.

Es zeigt sich, dass mit zunehmender Bestrahlungszeit der E-Modul der PMMASchichten gezielt gesteigert werden kann. Hierbei fällt auf, dass die Zunahme des EModuls schon durch 30 min UV-Bestrahlung von $3 \mathrm{MPa}$ auf 3, $7 \mathrm{GPa}$, also um mehrere Größenordnungen gesteigert werden kann. Desweiteren ist zu erkennen, dass die Kurve bei höheren Bestrahlungszeiten von ca. 200 min in eine Sättigung mit ca. 5 GPa geht. Somit reichen schon kurze UV-Bestrahlungszeiten von 30 min aus, um eine effiziente Steigerung des E-Moduls in den GPa-Bereich zu bewirken. In der Doktorarbeit von Britta Fuchs konnte zudem gezeigt werden, dass auch die Härte der Schichten durch ex-situ UV-Bestrahlung gezielt gesteigert werden kann [23]. Somit sei festgehalten, dass die Härte und der E-Modul dasselbe Verhalten aufweisen.

Durch die UV-Bestrahlung kommt es zu einer gesteigerten Anregung der Kettenvernetzung, welche bewirkt, dass die PMMA-Schichten härter werden und einen höheren E-Modul aufweisen. Dies ist durch die Verwendung von PMMA als Foto- 
lack in der Lithographie weitestgehend bekannt [81,87]. Mit Hilfe von NEXAFSUntersuchungen konnte gezeigt werden (siehe Abbildung 6.2), dass in den UVbestrahlten PMMA-Schichten im Vergleich mit einer unbestrahlten PMMA-Probe, die $\mathrm{C}=\mathrm{C}$-Doppelbindungen wieder deutlich abnehmen und die $\mathrm{C}=\mathrm{O}$-Doppelbindungen zunehmen. Hierbei wurden die Schichten ex-situ für 5 h UV-bestrahlt [23]. Die Abnahme der $\mathrm{C}=\mathrm{C}$-Doppelbindungen lässt sich dadurch erklären, dass dieser Bindungstyp durch die nachträgliche UV-Bestrahlung teilweise wieder aufgebrochen wird. Diese neu entstandenen freien Bindungen ermöglichen ein Quervernetzen, was sich in der gesteigerten Härte und dem gesteigertem E-Modul widerspiegelt. Ein weiterer Beweis für das Quervernetzen der Ketten ist auch in den SEC-Messungen zu finden. In der Arbeit von Britta Fuchs konnte gezeigt werden, dass die mittlere Molmasse einer für 1 h ex-situ UV-bestrahlten PMMA-Schicht im Vergleich mit einer unbehandelten Probe von $2,210^{3} \mathrm{~g} / \mathrm{mol}$ deutlich auf $5,110^{3} \mathrm{~g} / \mathrm{mol}$ anwächst [23].

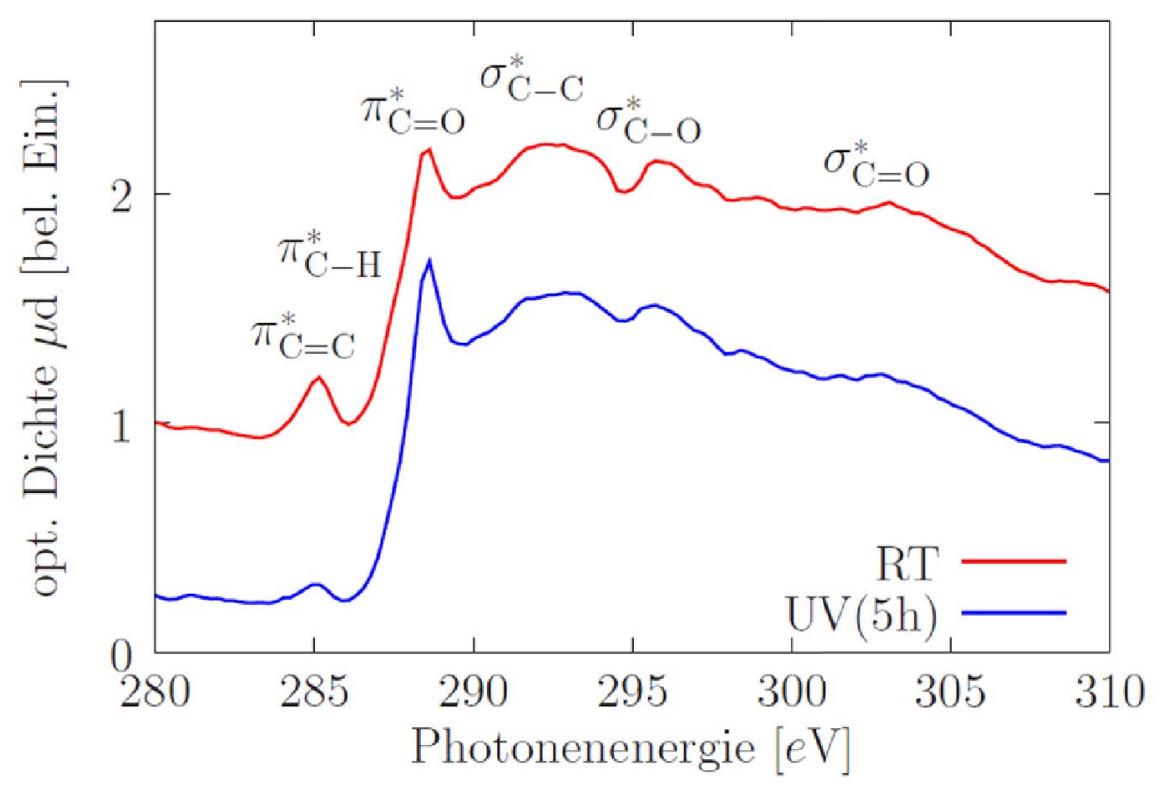

Abbildung 6.2: NEXAFS-Messungen einer unbehandelten PMMA-Schicht und einer UV-bestrahlten PMMA-Schicht. Hierbei wurde die PMMA-Schicht ex-situ für 5 h bestrahlt. In der Reduktion der $\mathrm{C}=\mathrm{C}$-Intensität ist das UV-induzierte Aufbrechen der Doppelbindung zu erkennen (Daten aus [23]).

Insgesamt bewirkt die UV-Bestrahlung eine gesteigerte Vernetzung der Ketten, was zu einem höherem E-Modul führt. Desweiteren behalten die PMMA-Schichten aber weiterhin eine glatte Oberfläche mit einer geringen Rauigkeit. Dies konnte durch rasterkraftmikroskopische Untersuchungen festgestellt werden.

Um den Einfluss der UV-induzierten Modifikation der PMMA-Schichten auf das Wachstum der Cu-Schichten zu untersuchen, wurden mehrere PMMA-Schichten in- 
situ für 30 min mit der UV-Lampe bestrahlt und anschließend mit unterschiedlichen $\mathrm{Cu}-$ Schichtdicken beschichtet. Anschließend wurden die Proben im AFM untersucht. In Abbildung 6.3 sind beispielhaft vier AFM-Aufnahmen von Proben mit CuSchichtdicken von $5 \mathrm{~nm}, 8 \mathrm{~nm}, 10 \mathrm{~nm}$ und $20 \mathrm{~nm}$ dargestellt. Es zeigt sich, dass nur die Probe mit einer $\mathrm{Cu}$-Schichtdicke von $5 \mathrm{~nm}$ keine Wellen aufweist. Somit reicht die UVBestrahlung insgesamt nicht aus, um die PMMA-Schichten ausreichend zu modifizieren, damit sie den Verspannungen in der Cu-Schicht widerstehen. Die Probe mit der $5 \mathrm{~nm}$ dicken $\mathrm{Cu}$-Schicht weist zwar keine Wellen auf, jedoch sei daran erinnert (Kapitel 5.1), dass auch unbestrahlte Proben bei einer $\mathrm{Cu}$-Schichtdicke von $5 \mathrm{~nm}$ (vergleiche Abbildung 5.1) aufgrund des vorliegenden Spannungsgleichgewichts zwischen Zug- und Druckspannungen keine Wellen zeigen.
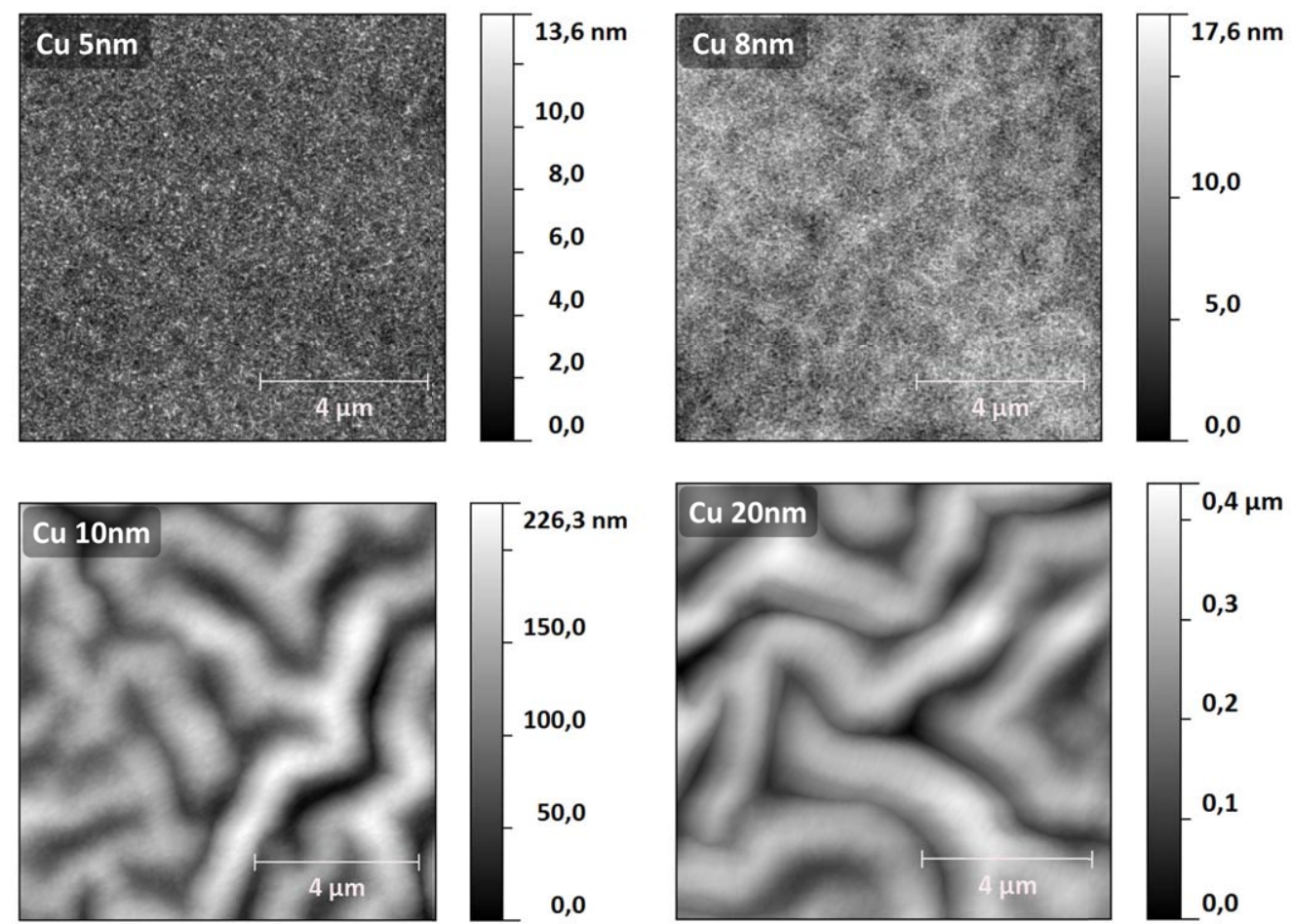

Abbildung 6.3: AFM-Aufnahmen von $500 \mathrm{~nm}$ dicken PMMA-Schichten, welche 30 min mit der UVLampe bestrahlt und anschließend mit $\mathrm{Cu}$ beschichtet wurden. Hierbei wurde die $\mathrm{Cu}$-Schichtdicke variiert. Es zeigt sich, dass nur bei der Cu-Schicht mit einer Schichtdicke von $5 \mathrm{~nm}$ keine Wellen auftreten.

Diese Cu-Schichtdicke $(5 \mathrm{~nm})$ sollte sich daher bestens eignen, um ein glattes PMMA/Cu-Schichtpaket herzustellen. Abbildung 6.4 zeigt ein Schichtpaket aus fünf Doppelschichten mit $450 \mathrm{~nm}$ dicken PMMA-Schichten und $5 \mathrm{~nm}$ dicken Cu-Schichten. Mit Hilfe des FIBs wurde ein Querschnitt der Probe erstellt, welcher dann mit 
einem REM untersucht werden konnte. Die unterste Schicht zeigt das Si-Substrat und die körnige oberste Schicht ist die Pt-Schutzschicht, welche vermeidet, dass beim Herstellen des Querschnittes das zu untersuchende Schichtpaket Schaden nimmt. Die hellen Schichten kennzeichnen die Cu-Schichten und die dunklen Schichten die PMMASchichten. Man erkennt, dass die $\mathrm{Cu}$-Schichten auf den UV-bestrahlten PMMASchichten viel glatter sind als im Vergleich zu den vorherigen Ergebnissen (s. Abb. 1.1). Auch wenn die UV-Modifizierung der PMMA-Schicht noch nicht ganz ausgereicht hat perfekt glatte Metallschichten zu erzeugen, so zeigt sich in Abb. 6.4 doch eine gewisse Tendenz zur Ausglättung bzw. Spannungskompensation.

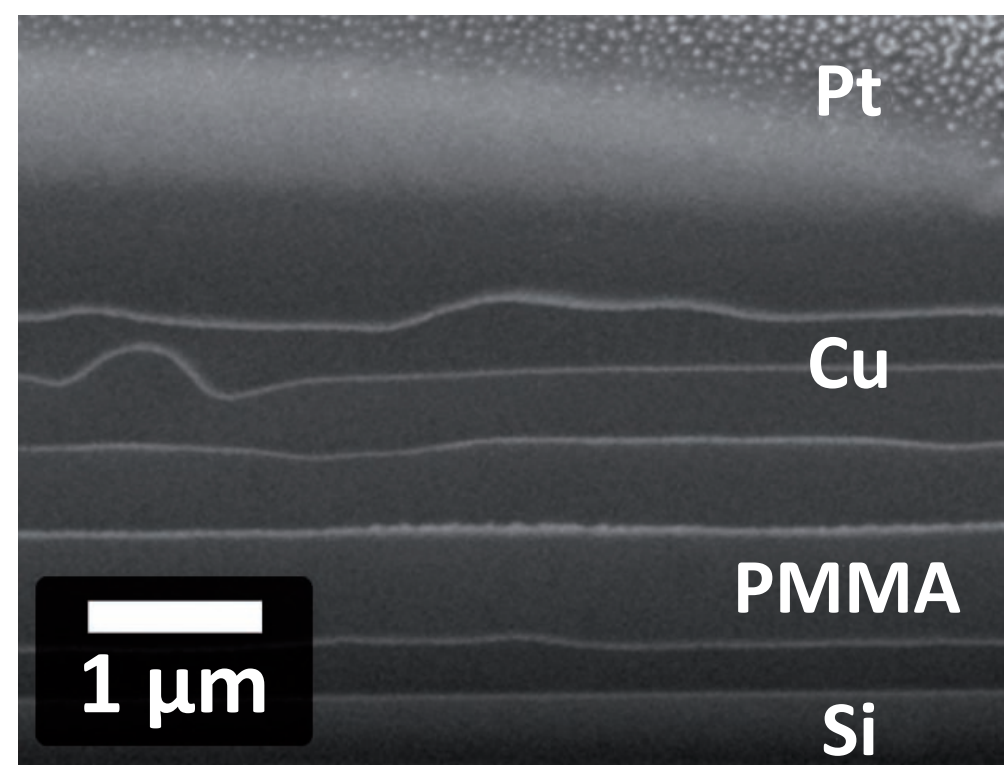

Abbildung 6.4: REM-Querschnittsaufnahme eines PMMA(450 nm)/Cu(5 nm)-Schichtpakets mit fünf Doppelschichten, wobei jede PMMA-Schicht in-situ für 30 min mit der UV-Lampe bestrahlt wurde. Die $\mathrm{Cu}$-Schichten sind im Vergleich zu vorherigen Ergebnissen (s. Abb. 1.1) viel glatter, zeigen jedoch teilweise noch kleine Wellen.

Zusammenfassend konnte gezeigt werden, dass die UV-Bestrahlung der PMMASchichten die Ketten zum Quervernetzen anregt, und somit der E-Modul der PMMASchichten gezielt gesteigert und eingestellt werden kann. Somit konnte ein PMMA/CuSchichtpaket hergestellt werden, welches im Vergleich zu den ersten Ergebnissen (s. Abb. 1.1), schon wesentlich glattere Cu-Schichten aufweist. Eine Steigerung der Bestrahlungszeit, was generell noch glattere Schichtpakete ermöglichen sollte durch insgesamt härtere Polymerschichten, ist insgesamt nicht zeiteffizient, um Schichtsysteme mit mehreren Doppelschichten herzustellen. Desweiteren ist nicht klar, wie tief die UV-Strahlung tatsächlich in die PMMA-Schicht dringt oder ob es hauptsächlich zu einer Modifikation der Oberfläche kommt. 


\subsubsection{Beeinflussung des Elastizitätsmoduls durch thermische Behandlung}

Eine weitere Möglichkeit die Vernetzung der Ketten anzuregen, um so den E-Modul der PMMA-Schichten zu steigern, ist eine thermische Behandlung der Schichten. Hierbei konnte in Zusammenarbeit mit Britta Fuchs gezeigt werden, dass die Härte von PMMASchichten durch thermische Behandlung gezielt gesteigert werden kann [23].

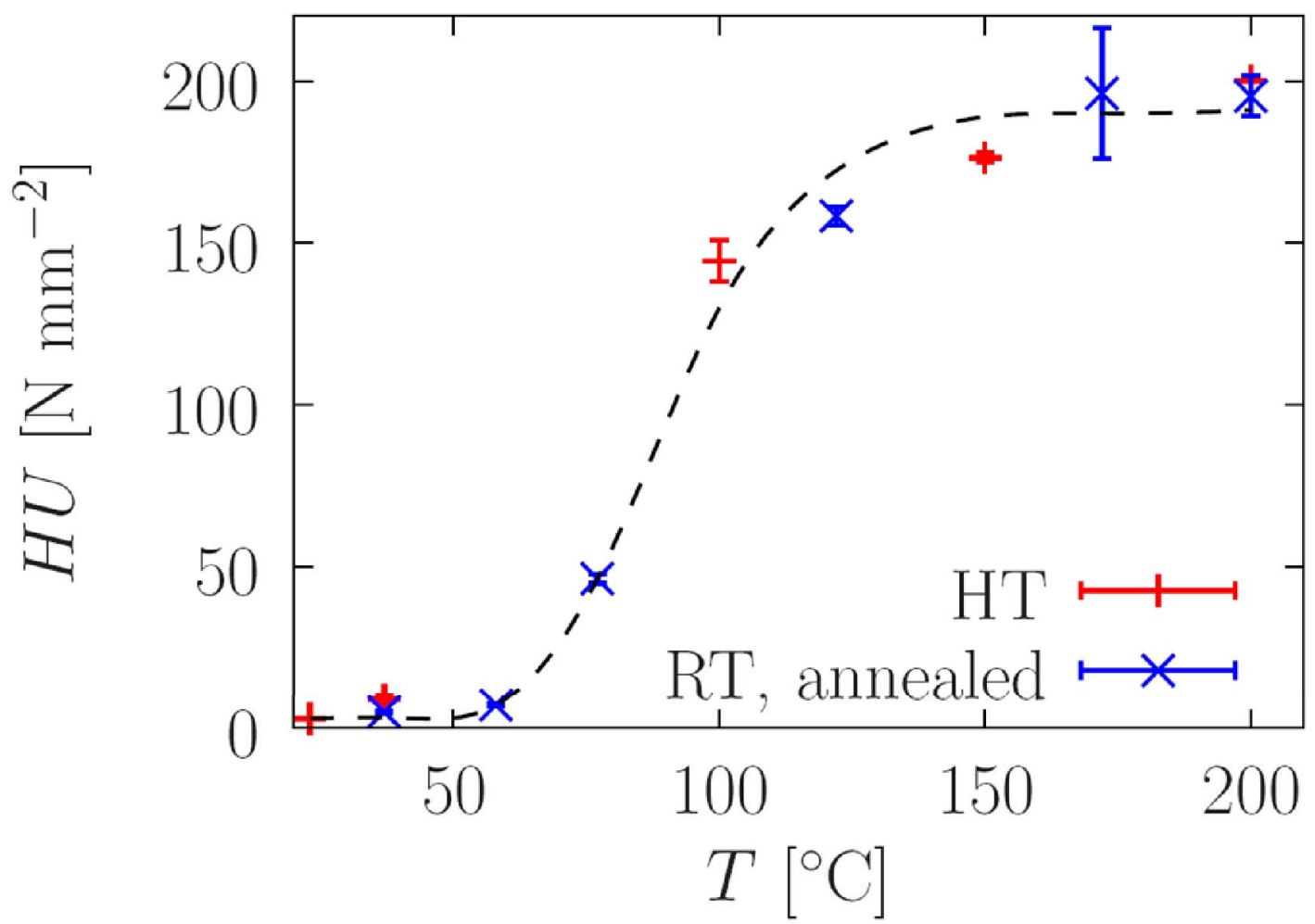

Abbildung 6.5: Abhängigkeit der Härte von der thermischen Behandlung der PMMA-Schichten. Mit steigender Temperatur nimmt die Härte der PMMA-Schichten zu, dabei macht es keinen Unterschied, ob während der Herstellung der Schichten geheizt wird oder die Schichten nach der Herstellung thermisch behandelt werden. Der Anstieg der Härte lässt sich über die angeregte Vernetzung der Ketten erklären (Daten aus [88]).

In Abbildung 6.5 ist die Abhängigkeit der Härte gegenüber der Temperatur dargestellt. Der Kurvenverlauf zeigt, dass schon bei einer Temperatur von $50 \mathrm{C}$ die Härte von anfänglich 2,8 N/mm² der PMMA-Schichten stark ansteigt und oberhalb von $100 \mathrm{C}$ in eine Sättigung bei einem Härtewert von $200 \mathrm{~N} / \mathrm{mm}^{2}$ gelangt. Desweiteren macht es keinen Unterschied, ob die Schichten direkt bei der Herstellung oder erst nachträglich thermisch behandelt wurden. Der E-Modul der Schichten zeigt dasselbe Verhalten. So 
besitzen PMMA-Schichten, welche bei einer Temperatur von $150 \mathrm{C}$ hergestellt wurden einen E-Modul von $5 \mathrm{GPa}$ [60].

Um nun zu untersuchen, welchen Einfluss die hohe Temperatur auf die Bindungsverhältnisse im PMMA hat, wurden NEXAFS-Messungen an einer unbehandelten PMMA-Schicht und im Vergleich dazu an einer bei $200 \mathrm{C}$ geheizten Probe gemacht. In Abbildung 6.6 ist anhand der NEXAFS-Messung zu erkennen, dass die $\mathrm{C}=\mathrm{C}$ Doppelbindungen zugenommen und die $\mathrm{C}=\mathrm{O}$-Bindungen abgenommen haben. Dies weist daraufhin, dass es zum Abdampfen der Seitengruppen aufgrund der hohen Temperatur kommt. Somit sollte eine Quervernetzung weiter angeregt worden sein, wodurch mehr $\mathrm{C}=\mathrm{C}$-Doppelbindungen ausgebildet werden konnten. Desweiteren ist anzunehmen, dass aufgrund der hohen Temperatur kleinere Kettenfragmente, die im Zuge der Laserdeposition in die PMMA-Schicht eingebaut wurden, zu einem Gewissen Grad ausgasen könnten, was zusätzlich zu einer Zunahme der Härte (des E-Moduls) des Polymers führen würde. Britta Fuchs konnte darüber hinaus anhand von SECMessungen zeigen, dass durch Steigerung der Temperatur auch die mittlere Molmasse der Schichten von 2,9 $10^{3} \mathrm{~g} / \mathrm{mol}$ auf $7,410^{3} \mathrm{~g} / \mathrm{mol}$ zunimmt, was das Quervernetzen der Ketten bestätigt [23].

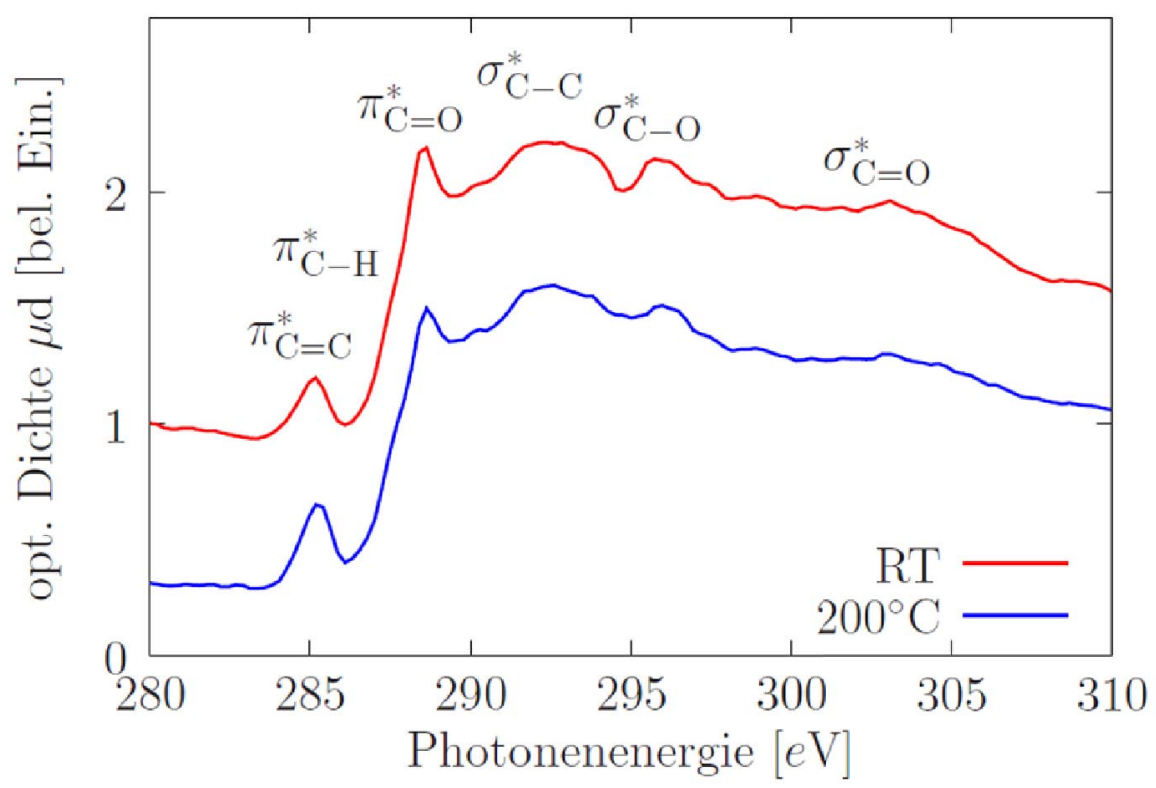

Abbildung 6.6: NEXAFS-Messungen von PMMA-Schichten, welche bei unterschiedlichen Temperaturen hergestellt wurden. Eine Zunahme der Intensität der C=C-Doppelbindungen spricht für ein Quervernetzen der Polymerketten (Daten aus [88]).

Um zu untersuchen, ob die Temperaturbehandlung der Schichten ausreicht, um glatte Cu-Schichten deponieren zu können, wurden bei 200 C PMMA-Schichten 
hergestellt. Anschließend ist zum einen direkt auf die noch heiße PMMA-Schicht $\mathrm{Cu}$ deponiert worden. In einem weiteren Experiment wurde die Cu-Schicht erst deponiert, nachdem die auf $200 \mathrm{C}$ aufgeheizte PMMA-Schicht auf Raumtemperatur abgekühlt war. In Abbildung 6.7 ist das Ergebnis dieser beiden Experimente anhand von REMAufnahmen zu erkennen. Deponiert man Cu auf eine noch heiße PMMA-Schicht (Bild a und b), so bilden sich große Wellen- und Blasenstrukturen auf der Probe aus. Kühlt man die PMMA-Schicht jedoch vor der Deposition des $\mathrm{Cu}$ erst wieder auf Raumtemperatur ab, so lässt sich das $\mathrm{Cu}$ glatt deponieren, ohne dass Wellen ausgebildet werden (Bild c und d). Das zeigt, dass ein Härten der Schichten durch Temperaturbehandlung möglich ist, aber dass die PMMA-Schichten erst im abgekühlten Zustand hart genug sind, um die Spannungen zu kompensieren. Bei noch höherer Temperatur sind die Schichten so weich, dass sich die Spannungen in den Cu-Schichten durch die Ausbildung der Wellenstrukturen komplett abbauen können. Allgemein ist bekannt, dass die Viskosität von Polymeren bei hohen Temperaturen sehr gering ist [89].
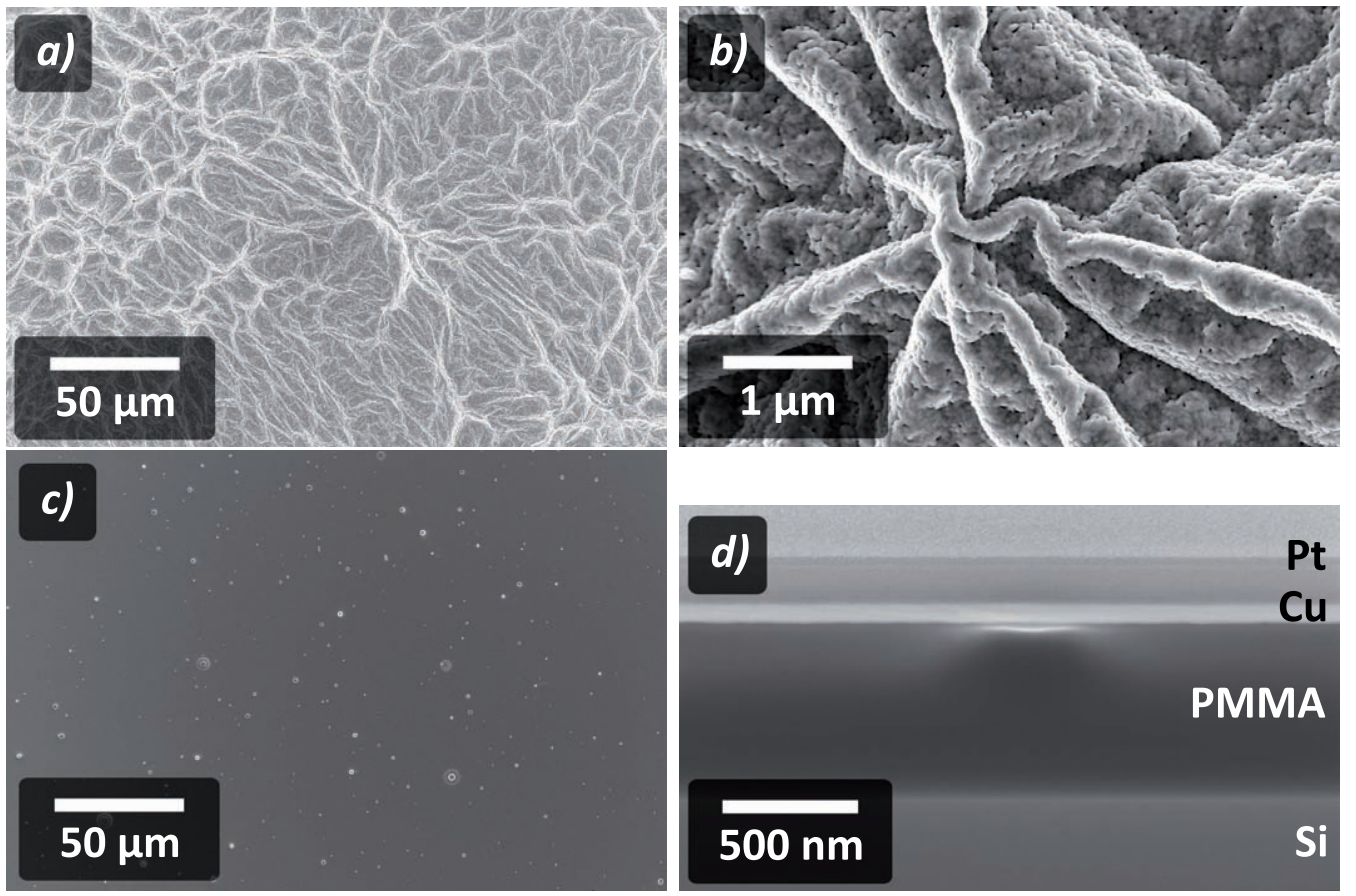

Abbildung 6.7: REM-Aufnahmen von PMMA/Cu-Schichten. In Bild (a) und (b) wurde direkt auf eine $200^{\circ} \mathrm{C}$ heiße PMMA-Schicht $50 \mathrm{~nm} \mathrm{Cu}$ deponiert. Dabei zeigt sich starke Wellenbildung. In Bild (c) und (d) wurde die PMMA-Schicht erst auf $200^{\circ} \mathrm{C}$ aufgeheizt und anschließend auf Raumtemperatur abgekühlt bevor $50 \mathrm{~nm} \mathrm{Cu}$ deponiert wurden. Die Probenoberfläche (c) und der Querschnitt (d) zeigen, dass die $\mathrm{Cu}$-Schichten glatt hergestellt werden konnten.

Der Kurvenverlauf in Abbildung 6.5 zeigt, dass schon bei Temperaturen um die $100 \mathrm{C}$ ein merklicher Anstieg in der Härtekurve zu verzeichnen ist. Um diesen 
markanten Anstieg genauer zu untersuchen, wurden mehrere PMMA-Schichten bei Temperaturen von $60 \mathrm{C}$ bis $120 \mathrm{C}$ für $1 \mathrm{~h}$ geheizt und anschließend für $1 \mathrm{~h}$ abgekühlt, bevor die $\mathrm{Cu}$-Schichten deponiert wurden. Die REM-Aufnahmen in Abbildung 6.8 zeigen allerdings, dass bei diesen Temperaturen immer noch große Wellenstrukturen vorhanden sind. Dies verdeutlicht, dass insbesondere die Abkühlzeit eine wichtige Rolle spielt, da der Vernetzungsprozess stark zeitabhängig zu sein scheint.
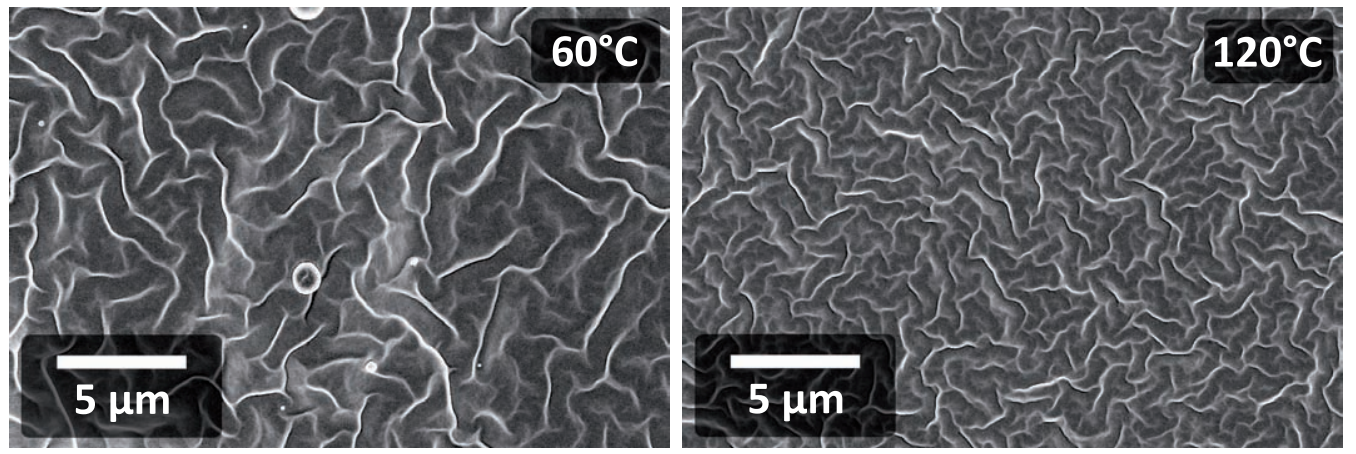

Abbildung 6.8: REM-Aufnahmen von geheizten PMMA/Cu-Schichten, bei denen die PMMASchichten für $1 \mathrm{~h}$ bei $60^{\circ} \mathrm{C}$ und $120^{\circ} \mathrm{C}$ geheizt und anschließend $1 \mathrm{~h}$ abgekühlt wurden, bevor die $\mathrm{Cu}$-Schicht deponiert wurde. Beide Proben zeigen Wellenbildung.

Deshalb wurde im Folgenden ein Schichtpaket aus drei PMMA(450 nm)/Cu(50 nm)Doppelschichten hergestellt, bei welchem jede PMMA-Schicht jeweils für $90 \mathrm{~min}$ auf $200 \mathrm{C}$ geheizt wurde. Vor der Deposition des $\mathrm{Cu}$ wurde jede PMMA-Schicht unterschiedlich lange abgekühlt, um zu überprüfen, bei welcher kritischen Abkühlzeit sich eine glatte $\mathrm{Cu}$-Schicht ausbildet. In Abbildung 6.9 ist eine REM-Aufnahme vom Querschnitt des Schichtpakets dargestellt. Die unterste Schicht bildet das SiSubstrat, danach folgt im Wechsel zuerst die PMMA-Schicht (dunkle Schicht) und anschließend die Cu-Schicht (helle Schicht). Abschließend ist die Pt-Deckschicht zu erkennen. Vom Substrat ausgehend wurde die erste PMMA-Schicht $5 \mathrm{~h}$ abgekühlt, bevor die $\mathrm{Cu}$-Schicht deponiert wurde. Es zeigt sich, dass die $\mathrm{Cu}-\mathrm{Schicht}$ sehr glatt ist und keine Wellen aufweist. Allerdings hat die erste PMMA-Schicht im Schichtpaket alle Temperaturbehandlungen (auch die der folgenden Schichten) zusätzlich erfahren, so dass diese erste PMMA-Schicht insgesamt dreimal geheizt wurde. Dies hat zu einem starken Schichtdickenverlust von ursprünglich $450 \mathrm{~nm}$ auf ca. $50 \mathrm{~nm}$ geführt. Die zweite PMMA-Schicht wurde für $14 \mathrm{~h}$ abgekühlt bevor die $\mathrm{Cu}$-Schicht deponiert wurde. Auch diese ist sehr glatt gewachsen. Die letzte PMMA-Schicht wurde für $3 \mathrm{~h}$ abgekühlt. Hier zeigt sich, dass die Cu-Schicht kleine Wellen aufweist, was dafür spricht, dass eine Abkühlzeit von $3 \mathrm{~h}$ nicht ausreicht, um ein vollständiges Quervernetzen anzuregen. Weiterhin ist zu beobachten, dass nur die für $14 \mathrm{~h}$ abgekühlte PMMA-Schicht als einzige 
keinen Schichtdickenverlust aufweist. Dies zeigt, dass eine lange Abkühlzeit notwendig ist, um sowohl eine glatte $\mathrm{Cu}$-Schicht deponieren zu können als auch die Schichtdicke der PMMA-Schicht konstant halten zu können.

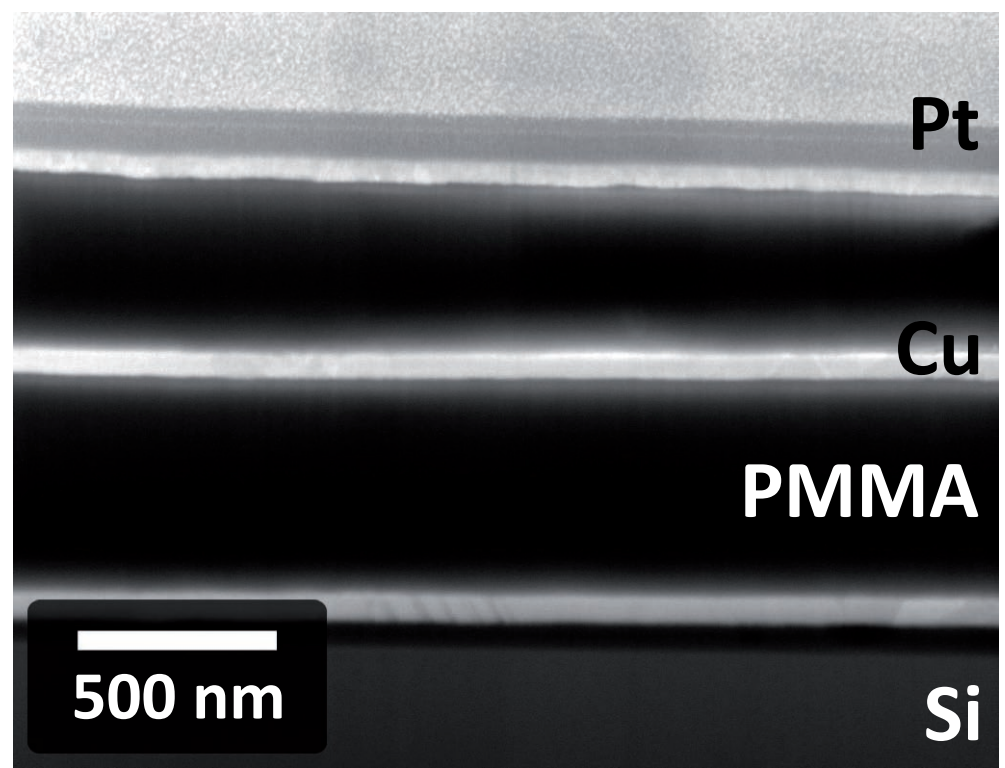

Abbildung 6.9: REM-Querschnittsaufnahme einer $\mathrm{PMMA}(450 \mathrm{~nm}) / \mathrm{Cu}(50 \mathrm{~nm})$-Multischicht mit drei Doppelschichten. Die PMMA-Schichten wurden bei einer Temperatur von $200^{\circ} \mathrm{C}$ deponiert. Anschließend wurde die Probe abgekühlt und die $\mathrm{Cu}$-Schicht deponiert. Hierbei wurden unterschiedliche Abkühlzeiten eingestellt (1. Cu-Schicht 5 h, 2. Cu-Schicht 14h, 3. Cu-Schicht 3 h). Alle drei $\mathrm{Cu}$-Schichten sind glatt aufgewachsen, jedoch haben die PMMA-Schichten aufgrund der thermischen Behandlung einen großen Schichtdickenverlust erlitten.

Zusammenfassend konnte gezeigt werden, dass durch eine Temperaturbehandlung der PMMA-Schichten der E-Modul und die Härte der Schichten gezielt eingestellt werden kann. Desweiteren ist es somit auch möglich, glatte $\mathrm{Cu}$-Schichten auf diese PMMA-Schichten zu deponieren. Allerdings ist dies nur möglich, wenn die PMMASchichten auf Raumtemperatur abgekühlt sind, da der Vernetzungsprozess stark zeitabhängig zu sein scheint. 


\subsubsection{Beeinflussung des Elastizitätsmoduls durch Verwendung eines Vernetzers}

Eine weitere Möglichkeit den E-Modul der PMMA-Schichten zu steigern, kann durch die Verwendung eines Vernetzers erzielt werden. Wie schon in Kapitel 4.3 beschrieben eignen sich hierfür die Polymere BisDMA und PC. Hierbei wurden im Folgenden nur PMMA/PC-Mischschichten untersucht, da die BisDMA-Targets nur durch ein sehr aufwendiges Herstellungsverfahren gewonnen werden konnten.
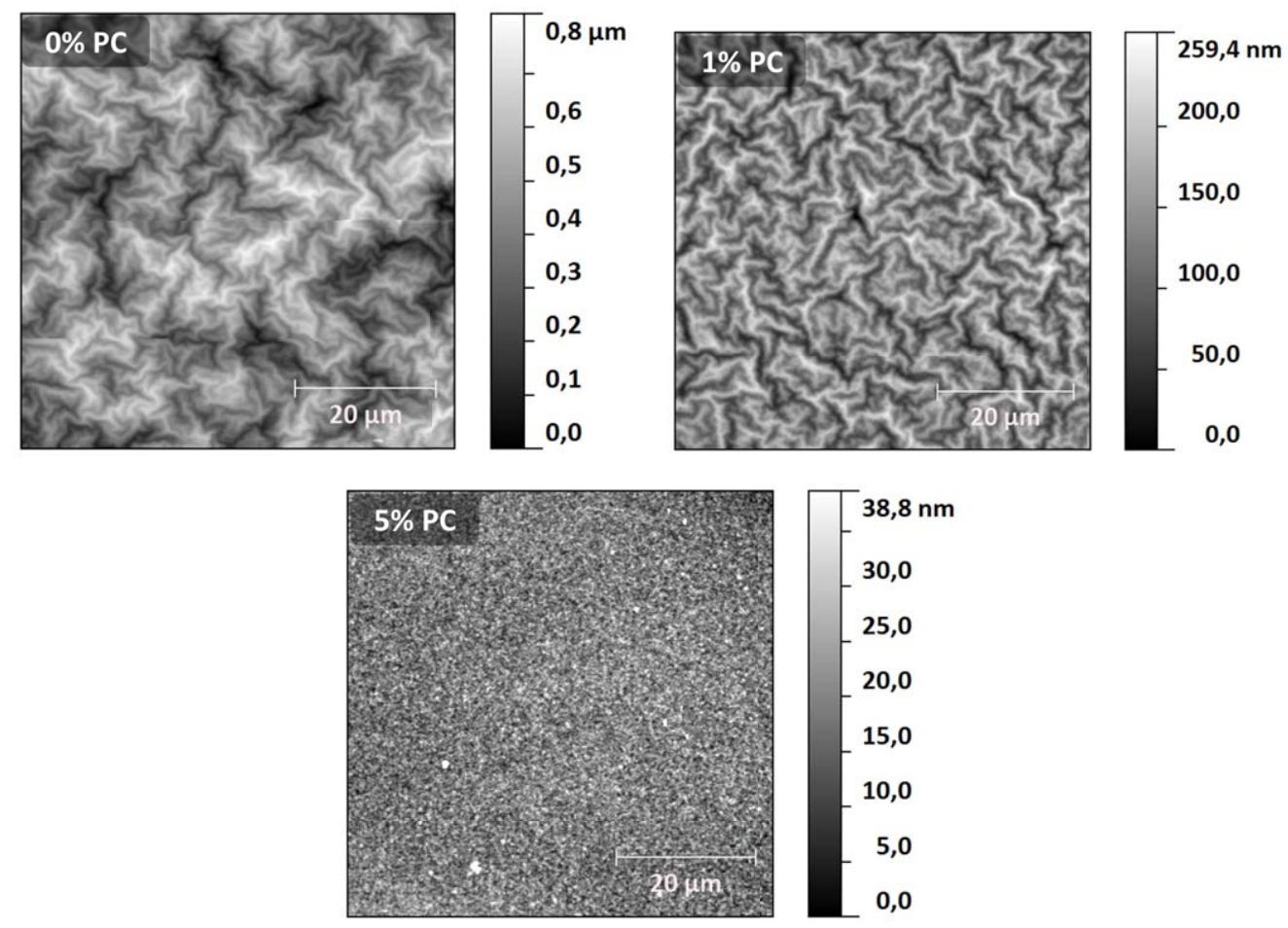

Abbildung 6.10: AFM-Aufnahmen von PMMA/PC-Mischschichten mit unterschiedlichem Anteil an $\mathrm{PC}(0 \%, 1 \%, 5 \%)$, auf welche $50 \mathrm{~nm} \mathrm{Cu}$ deponiert wurde. Mit zunehmendem Anteil an PC, von links nach rechts, werden die Wellen kleiner und verschwinden sogar komplett bei einem Anteil von $5 \% \mathrm{PC}$.

Um zu untersuchen, ob die Steigerung des E-Moduls der PMMA/PC-Mischschichten ausreicht, um glatte $\mathrm{Cu}$-Schichten darauf deponieren zu können, wurden mehrere PMMA/PC-Mischschichten mit unterschiedlichem Anteil an PC hergestellt und $50 \mathrm{~nm}$ $\mathrm{Cu}$ darauf deponiert. In Abbildung 6.10 sind beispielhaft drei AFM-Aufnahmen von (PMMA/PC)/Cu-Schichten mit einem PC-Anteil von $0 \%$ (links), $1 \%$ (Mitte) und $5 \%$ (rechts) dargestellt. Hierbei sind auf der Schicht mit einem Anteil von $0 \%$ PC die Wellen ganz normal aufgewachsen ohne jeglichen Einfluss von PC. Die Schicht mit 
einem Anteil von 1\% PC weist dazu im Vergleich schon deutlich kleinere Wellen auf und bei der Schicht mit einem Anteil von 5\% PC sind bereits keine Wellen mehr vorhanden.

Um das Verhalten der Wellenlängen und Amplituden besser veranschaulichen zu können, sind in Abbildung 6.11 Höhenprofile der obigen AFM-Aufnahmen dargestellt. Hierbei wird deutlich, dass schon der Einfluss von 1\% PC die Wellenlänge von fast $14 \mu \mathrm{m}$ ( $0 \%$ PC) auf $2 \mu \mathrm{m}$ verkleinert. Die Amplitude nimmt dabei von fast $500 \mathrm{~nm}$ (0\% PC) auf $100 \mathrm{~nm}$ ab. Das Höhenprofil der Schicht mit einem Anteil von $5 \%$ PC zeigt nur noch eine minimale Rauigkeit und keine Wellen mehr.

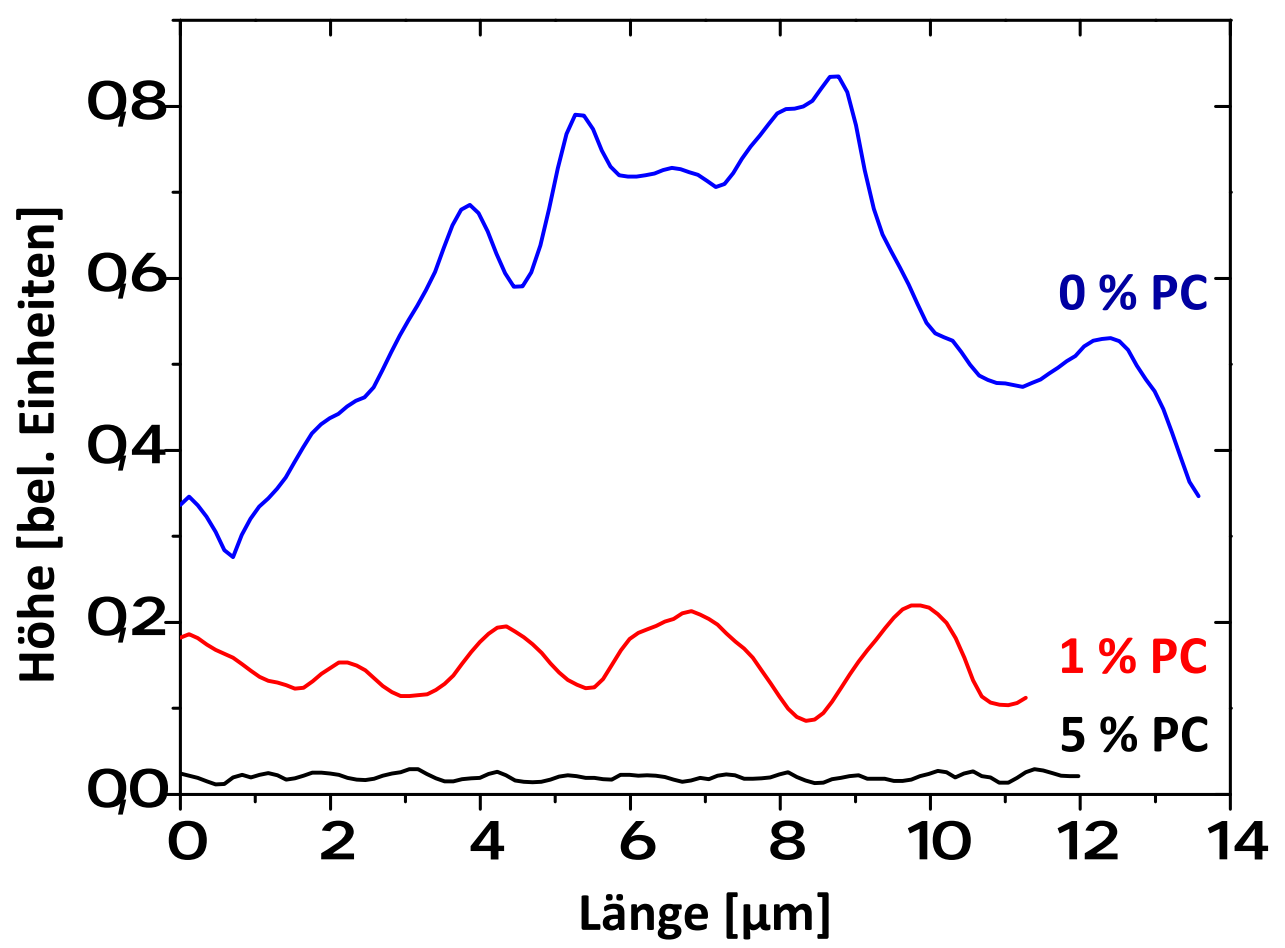

Abbildung 6.11: Höhenprofile der PMMA/PC-Mischschichten mit PC-Anteilen von 0\%, 1\%, 5\%, auf welche $50 \mathrm{~nm} \mathrm{Cu}$ deponiert wurde. Es zeigt sich, dass mit zunehmendem Anteil an PC die Wellenlänge und die Amplitude der (PMMA/PC)/Cu-Schichten stark reduziert werden und bei einem Anteil von $5 \%$ PC komplett verschwinden.

Betrachtet man die RMS-Rauigkeit der (PMMA/PC)/Cu-Schichten in Abhängigkeit vom Anteil an PC (siehe Abbildung 6.12), so zeigt sich ein starker Abfall der Kurve schon innerhalb eines sehr geringen Anteils an PC. Durch Extrapolation ergibt sich, dass schon bei einem Anteil von nur 1,4\% PC die RMS-Rauigkeit von anfangs $130 \mathrm{~nm}$ auf $4 \mathrm{~nm}$ reduziert wird. Dies zeigt, wie groß der Einfluss von schon kleinen Mengen 
PC auf die Ausbildung der Wellen ist. Dieser starke Einfluss von PC konnte bereits in Kapitel 4.3 in Abbildung 4.15 anhand der Steigerung des E-Moduls in den PMMA/PCMischschichten gezeigt werden. Der E-Modul wird dabei von anfangs $3 \mathrm{MPa}$ für eine reine PMMA-Schicht (0\% PC) durch Dazulegieren von 1\% PC auf einen E-Modul von 0, $9 \mathrm{GPa}$ gesteigert. Bei einem Anteil von 5\% PC erreicht der E-Modul einen Wert von 1,2 GPa. Somit lässt sich abschätzen, dass für einen Anteil von 1,4\% PC der E-Modul ungefähr einen Wert von $1 \mathrm{GPa}$ besitzen sollte. Dies ist ein erster Hinweis darauf, dass Polymerschichten mit einem E-Modul von 1 GPa den Spannungen der CuSchichten widerstehen können, und somit die Herstellung glatter $\mathrm{Cu}$-Schichten auf den Polymerschichten möglich wird.

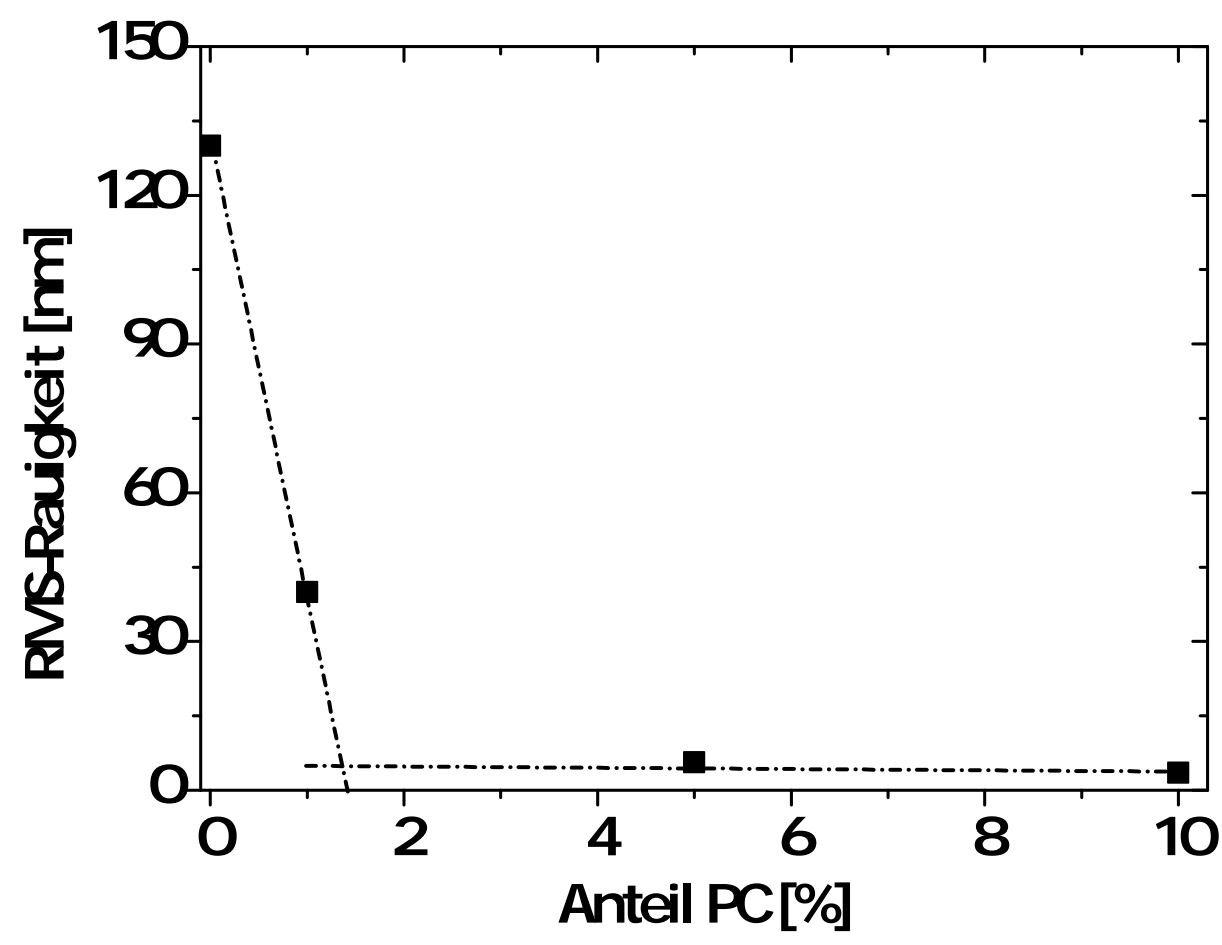

Abbildung 6.12: RMS-Rauigkeit der (PMMA/PC)/Cu-Schichten in Abhängigkeit vom Anteil an PC. Es zeigt sich durch Extrapolation, dass bei einem Anteil von 1,4\% PC die RMS-Rauigkeit von anfangs $130 \mathrm{~nm}$ auf nur noch $4 \mathrm{~nm}$ sinkt.

Unter Verwendung der Formel (2.18), welche in Kapitel 2 hergeleitet wurde, lässt sich zusätzlich die kritische Spannung $\sigma_{k r i t}$ bestimmen. Dabei wird der E-Modul für die Metallschicht $E_{M}$, in diesem Fall für die Cu-Schicht als Bekannt angenommen mit einem Wert von $E_{M}=115 \mathrm{GPa}$. 


$$
\sigma_{k r i t} \quad 0,548\left(E_{M}\right)^{\frac{1}{3}}\left(E_{P}\right)^{\frac{2}{3}}
$$

Das Verhältnis der Elastizitätsmoduln der beiden Komponenten gibt einen ersten Rückschluss auf die kritische Spannung, welche zwischen den beiden Materialien herrscht und welche unter anderem für die Wellenbildung verantwortlich ist.

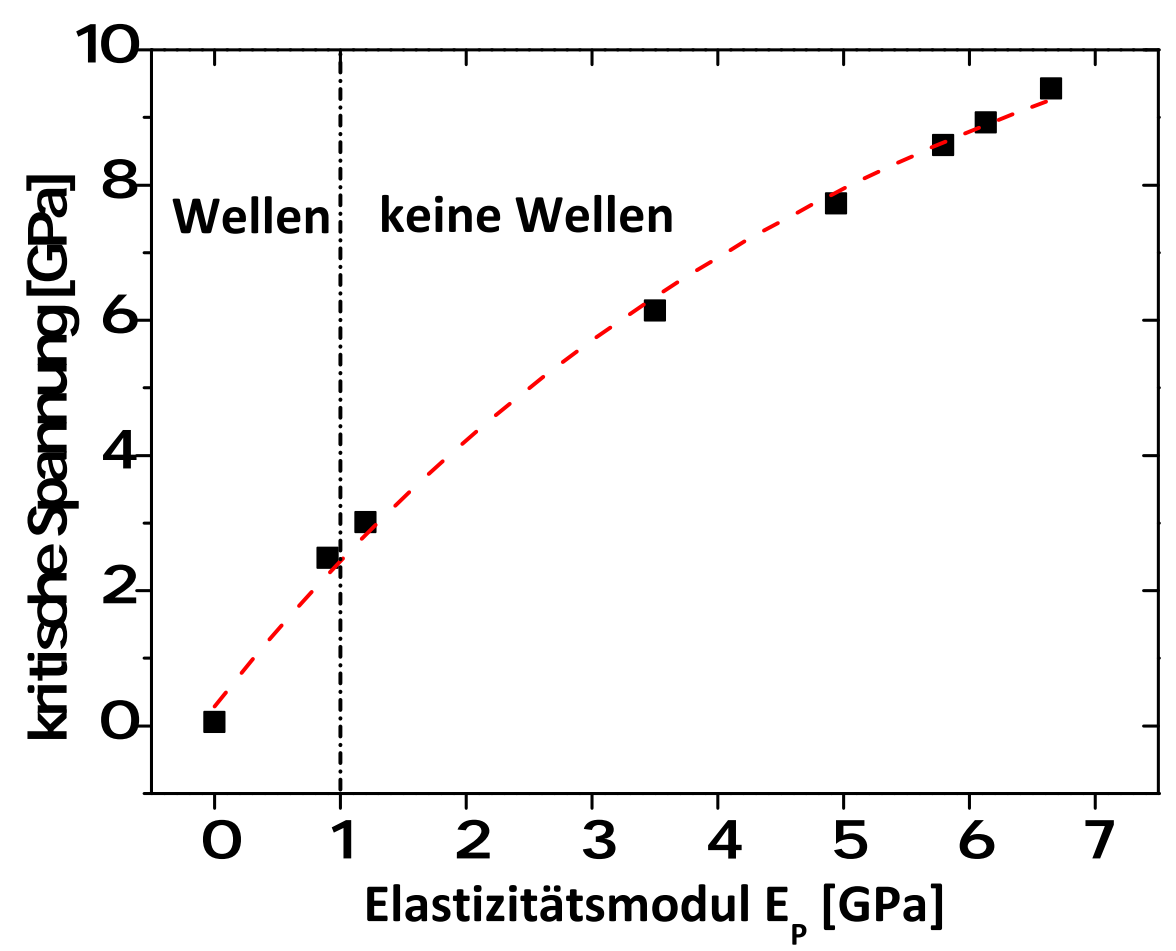

Abbildung 6.13: Kritische Spannung in Abhängigkeit vom Elastizitätsmodul der PC/PMMAMischschichten. Ein Übergang von welligen zu glatten $\mathrm{Cu}$-Schichten ist für Polymerschichten mit einem E-Modul von $1 \mathrm{GPa}$ abgeschätzt worden. Damit lässt sich eine kritische Spannung von ungefähr 2,6 GPa abschätzen.

In Abbildung 6.13 ist die kritische Spannung gegen den E-Modul der PMMA/PCMischschichten $E_{P}$ aufgetragen. Hierbei findet der Übergang von welligen zu glatten $\mathrm{Cu}-$ Schichten, wie obig postuliert, bei einem E-Modul Wert der Polymerschicht von $1 \mathrm{GPa}$ statt. Dies erhärtet den Verdacht, dass Polymerschichten mit einem E-Modul von mindestens $1 \mathrm{GPa}$ den Spannungen in der Cu-Schicht widerstehen können. Dadurch lässt sich weiterhin abschätzen, dass bei einem E-Modul von $1 \mathrm{GPa}$ eine kritische Spannung von 2,6 GPa zwischen beiden Materialien vorliegt. Aus früheren Arbeiten ist bekannt, dass die Spannungen in laserdeponierten Metallschichten um die $12 \mathrm{GPa}$ 
betragen [65]. Hierzu sei nochmals auf Abbildung 5.14 verwiesen in der eine Spannungsmessung an Ag und Permalloy dargestellt ist. Unter normalen Herstellungsbedingungen (bei einem Kammerdruck von $10{ }^{7}$ mbar) betragen die Spannungen in den Schichten 0,3 GPa für Ag und 1,8 GPa für Permalloy. Dies zeigt, dass der berechnete Wert in der richtigen Größenordnung liegt. Die leichte Abweichung nach oben könnte eventuell auf einen vorliegenden Substrateinfluss hinweisen, da die PMMA-Schichtdicke nur $6 \mu \mathrm{m}$ auf einem Si-Substrat betrug.

Insgesamt hat sich gezeigt, dass ein geringer Anteil von $1,4 \% \mathrm{PC}$ in den (PMMA/PC)/Cu-Schichten ausreicht, so dass glatte $\mathrm{Cu}$-Schichten deponiert werden können. Dies entspricht einem E-Modulwert der Polymerschicht von 1 GPa bei welchem der Übergang von welligen zu glatten Cu-Schichten stattfindet. Dadurch ließ sich ein Wert für die kritische Spannung von 2,6 GPa abschätzen.

\subsection{Multischichtsysteme}

In diesem Kapitel werden die verschiedenen in dieser Arbeit hergestellten Multischichtsysteme vorgestellt. Dabei wird zunächst auf die Optimierung des Herstellungsprozesses eingegangen. Neben dem System PMMA/Cu werden die Systeme BisDMA/Cu und PC/Cu näher betrachtet, welche auch ausführlich auf ihre mechanischen Eigenschaften untersucht worden sind.

\subsubsection{Optimierung zur Herstellung periodischer Polymer/Metall-Schichtpakete}

Die gepulste Laserdeposition bietet die Möglichkeit, sowohl Polymerschichten als auch Metallschichten in einem Herstellungsprozess zu erzeugen. Um glatte Schichten zu deponieren, ist besonders bei den Polymerschichten eine sehr geringe Energiedichte von $77 \mathrm{~mJ} / \mathrm{cm}^{2}$ notwendig. Bei den Metallschichten wird hingegen eine vergleichsweise hohe Energiedichte von $3 \mathrm{~J} / \mathrm{cm}^{2}$ benötigt, um eine optimale Herstellungsrate zu erhalten. Bisher konnte die niedrige Energiedichte nur durch ein manuelles Verschieben der Linse in Richtung optischer Achse erreicht werden. Das Verschieben der Linse in dieser Richtung hatte eine Aufweitung des Brennflecks am Target zur Folge. Somit wurde die Energie über eine größere Fläche verteilt, was zur Minimierung der Energiedichte führte. Um ein Multischichtsystem mit mehreren Doppelschichten herzustellen, musste 
also nach jeder Einzelschicht die Linse manuell verschoben werden, was den gesamten Herstellungsprzoss sehr zeitaufwendig machte.

Um den Herstellungsprozess zu Optimieren wurde für diese Arbeit ein neues Linsensystem aufgebaut. Hierbei wurden zwei Linsen mit gleicher Brennweite von $f_{1,2}=$ $70 \mathrm{~cm}$ ausgewählt. Nach dem Linsengesetz addieren sich die reziproken Brennweiten [90].

$$
\frac{1}{f}=\frac{1}{f_{1}}+\frac{1}{f_{2}} \quad \frac{d}{f_{1} f_{2}}
$$

Dabei bezeichnet $d$ den Abstand zwischen den beiden Linsen. Wenn der Abstand zwischen den beiden Linsen sehr viel kleiner ist als deren Brennweite $\left(d<<f_{1}\right.$ und $d<<f_{2}$ ), so kann der letzte Term der Gleichung vernachlässigt werden. Dieser Fall trifft hier zu, da beide Linsen direkt hintereinander im Strahlengang positioniert sind, und es resultiert eine Brennweite von $f=35 \mathrm{~cm}$. Bei dieser Brennweite ist der Laserstrahl auf das Target fokussiert, und es lassen sich, weiterhin hohe Energiedichten für die Metalldeposition einstellen. Desweiteren wurde eine der beiden Linsen auf einem Lineartisch befestigt, welche ein automatisches Verfahren in und aus dem Strahlengang ermöglicht. Somit lässt sich mit nur einer Linse im Strahlengang die Brennweite auf $f=70 \mathrm{~cm}$ einstellen, was eine Defokussierung des Laserstrahls am Target bewirkt, um auch geringere Energiedichten für die Polymerdeposition einzustellen.

Abschließend wurden die Parameter für eine optimale Rate und eine minimale Dropletdichte für beide Materialien neu bestimmt. Durch das neue Linsensystem ist es nun möglich, ein komplettes Schichtpaket in einem Herstellungsprozess ohne Unterbrechung zu deponieren. Dies hat einerseits eine erhebliche Zeitersparnis ermöglicht und andererseits die Qualität der Schichtpakete gesteigert.

\subsubsection{PMMA/Cu-Multischichtsysteme}

Das Schichtsystem PMMA/Cu ist in den vorherigen Kapiteln grundlegend untersucht worden. Hier hat sich gezeigt, dass es zur Wellenbildung kommt und die Wellenlänge über die $\mathrm{Cu}$ - als auch die PMMA-Schichtdicke in ihrer Größe eingestellt werden kann. Jedoch gibt es nur einen sehr kleinen Schichtdickenbereich, indem eine Cu-Schicht glatt auf einer PMMA-Schicht aufwächst. Dieser Bereich befindet sich zwischen $5 \mathrm{~nm}$ und $10 \mathrm{~nm} \mathrm{Cu-Schichtdicke.} \mathrm{Hier} \mathrm{befinden} \mathrm{sich} \mathrm{die} \mathrm{anliegenden} \mathrm{Spannungen} \mathrm{(Zug-}$ und Druckspannung) im Gleichgewicht. Allerdings haben TEM-Aufnahmen gezeigt, 


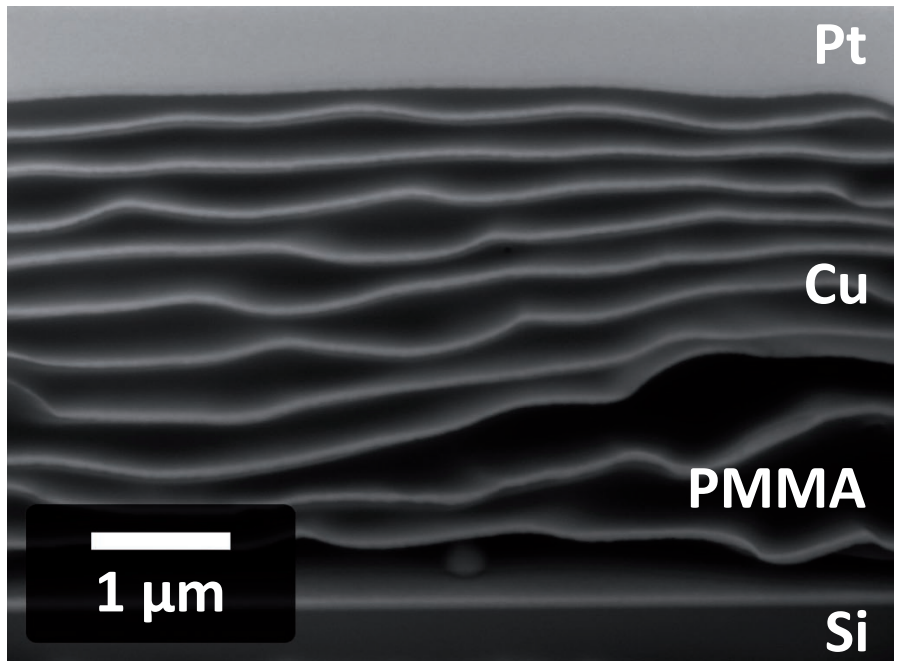

Abbildung 6.14: REM-Querschnittsaufnahme eines PMMA(450 nm)/Cu(50 nm)-Schichtpakets mit 11 Doppelschichten. Die Cu-Schichten zeigen eindeutig Wellenbildung.

dass die $\mathrm{Cu}$-Schichten unterhalb von $10 \mathrm{~nm} \mathrm{Cu}$-Schichtdicke noch nicht komplett geschlossen sind. Dies führt dazu, dass das Schichtdicken-Fenster, in dem es möglich wäre, ein Schichtpaket mit glatten $\mathrm{Cu}$-Schichten zu erzeugen, sehr klein ist, was die experimentelle Umsetzung sehr schwierig macht.

Eine weitere Idee besteht darin, auch die PMMA-Schichtdicke so klein zu wählen, dass ein großer Substrateinfluss vorliegt. Dies führt, wie Kapitel 5.2 gezeigt hat, zu einer „Pseudo-Härtung“ des Polymers. In Abbildung 6.15 (linkes Bild) ist ein Querschnitt eines PMMA/Cu-Schichtpakets mit 20 Doppelschichten und einer Einzelschichtdicke von jeweils $20 \mathrm{~nm}$ dargestellt. Es zeigt sich, dass die $\mathrm{Cu}$-Schichten im Vergleich zu den vorherigen Ergebnissen (s. Abb. 6.14) schon wesentlich glatter sind. Insbesondere die ersten drei Cu-Schichten, vom Substrat ausgehend, sind sehr glatt. Die AFMAufnahme desselben Schichtpakets (s. Abb. 6.15 rechts) zeigt jedoch kleine Wellen auf der Oberfläche. Somit reicht der Substrateinfluss nicht gänzlich aus, um den Spannungen in den $\mathrm{Cu}$-Schichten entgegenzuwirken.

Aufgrund des geringen Elastizitätsmoduls der PMMA-Schichten können also nur bedingt glatte $\mathrm{Cu}$-Schichten deponiert werden. Auch eine Modifikation der PMMASchichten, welche den Elastizitätsmodul der Schichten erhöhen, reicht nicht aus. Hier hat sich gezeigt, dass es prinzipiell möglich ist, durch eine thermische Behandlung der PMMA-Schicht eine glatte $\mathrm{Cu}$-Schicht deponieren zu können. Jedoch ist es nicht möglich, dadurch ein periodisches Schichtpaket mit konstanter Doppelschichtdicke 


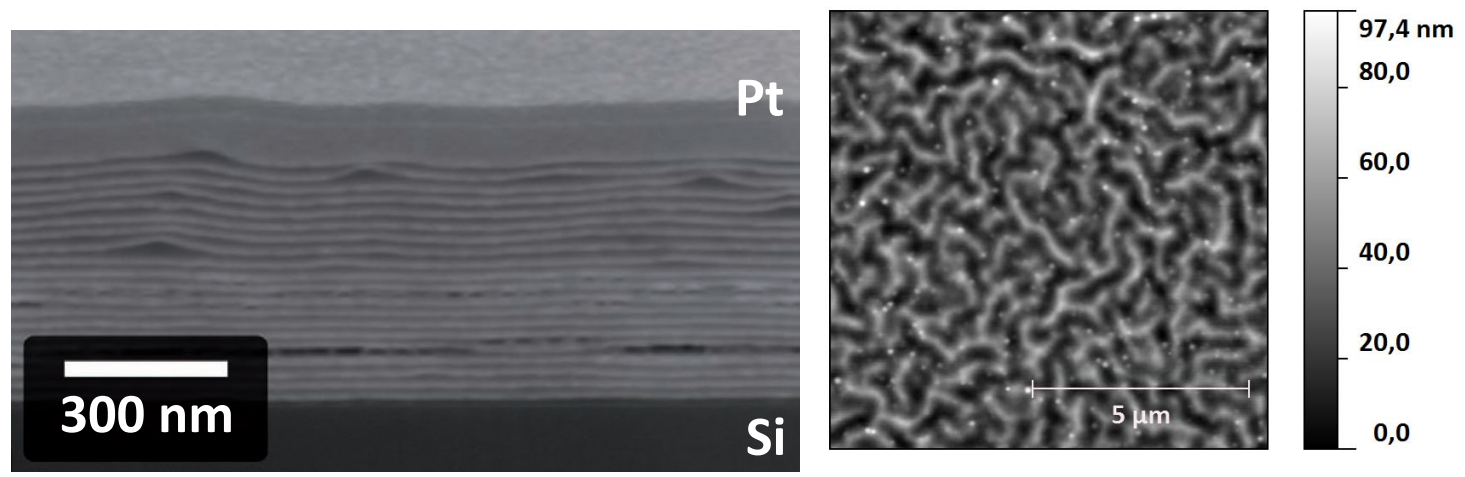

Abbildung 6.15: REM-Querschnittsaufnahme einer $\operatorname{PMMA}(20 \mathrm{~nm}) / \mathrm{Cu}(20 \mathrm{~nm})$-Multischicht mit 20 Doppelschichten (links). Die hellen Schichten bestehen aus $\mathrm{Cu}$, die dunklen aus PMMA. Insgesamt lässt sich erkennen, dass die Cu-Schichten trotz des Substrateinflusses nicht komplett glatt sind. Auch die AFM-Aufnahme der Oberfläche desselben Schichtpakets zeigt kleine Wellen (rechts).

zu erzeugen, da aufgrund der thermischen Behandlung die Schichtdicken nicht mehr kontrolliert werden können.

Insgesamt eignet sich das PMMA also nur bedingt dazu, um periodische Polymer/Metall-Schichtpakete zu erzeugen (siehe Abbildung 6.14). Der Grund dafür liegt einzig und allein in dem geringen Elastizitätsmodul, welcher den Spannungen im $\mathrm{Cu}$ nicht entgegenwirken kann.

\subsubsection{BisDMA/Cu-Multischichtsysteme}

BisDMA-Schichten besitzen, im Vergleich zu den PMMA-Schichten, einen deutlich höheren Elastizitätsmodul von $6 \mathrm{GPa}$. Um zu untersuchen, ob glatte $\mathrm{Cu}$-Schichten darauf deponiert werden können, wurde in einem ersten Experiment ein Schichtpaket aus beiden Komponenten hergestellt. Hierbei wurde die $\mathrm{Cu}$-Schichtdicke variiert $(40 \mathrm{~nm}, 80 \mathrm{~nm}, 120 \mathrm{~nm}$ und $160 \mathrm{~nm})$, um zu überprüfen bis zu welcher Schichtdicke die $\mathrm{Cu}$-Schichten glatt auf den $300 \mathrm{~nm}$ dicken BisDMA-Schichten aufwachsen.

In Abbildung 6.16 ist eine REM-Querschnittsaufnahme von dem Schichtpaket dargestellt. Es zeigt sich, dass sogar die dickste $\mathrm{Cu}$-Schicht, bei der die höchsten Druckspannungen zu erwarten sind, glatt auf der BisDMA-Schicht aufgewachsen ist. Der hohe Elastizitätsmodul der BisDMA-Schichten reicht aus, wie in Abschnitt 6.1.3 mit der Bestimmung einer kritischen Spannung bzw. eines kritischen E-Moduls gezeigt hat, um den Spannungen im Cu zu widerstehen. Dadurch eignet sich BisDMA hervorragend, um glatte, periodische BisDMA/Cu-Schichtpakete zu erzeugen. Dies 


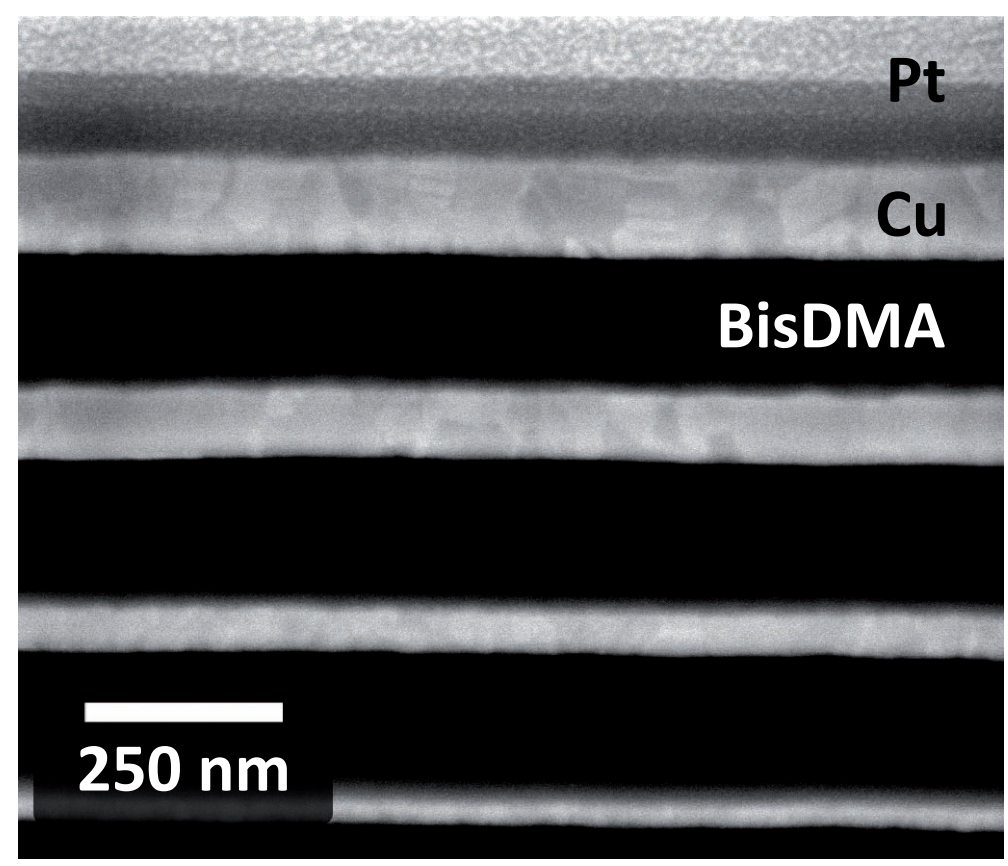

Abbildung 6.16: REM-Querschnittsaufnahme eines $\operatorname{BisDMA}(300 \mathrm{~nm}) / \mathrm{Cu}-\mathrm{Schichtpakets}$, wobei die $\mathrm{Cu}$-Schichtdicke variiert wurde $(40 \mathrm{~nm}, 80 \mathrm{~nm}, 120 \mathrm{~nm}$ und $160 \mathrm{~nm})$. Alle Schichten zeichnen sich durch sehr glatte Grenzflächen aus.

bestätigt auch die TEM-Aufnahme einer $\operatorname{BisDMA}(300 \mathrm{~nm}) / \mathrm{Cu}(30 \mathrm{~nm})-$ Multischicht in Abb. 6.17 in der alle Cu-Schichten glatt sind.

In Zusammenarbeit mit Inga Knorr und Timo Wuttke wurden mehrere BisDMA/CuSchichtpakete mechanisch untersucht [60]. Ziel der Untersuchungen war es, durch mechanische Untersuchungen der Einzelkomponenten als auch der Schichtpakete, Aussagen über die Grenzflächeneigenschaften des Schichtsystems zu gewinnen. Dafür wurden zum einen Berkovich-Indents an den Multischichten vorgenommen (siehe Abbildung 6.18) als auch Mikrodruckversuche an Säulen durchgeführt (siehe Abbildung $6.19)$.

Die mechanischen Untersuchungen der Einzelkomponenten haben ergeben, dass BisDMA mechanisch wesentlich langzeitstabiler ist als eine PMMA-Schicht. Das heißt, sowohl die Härte als auch der E-Modul der BisDMA-Schichten verändern sich nicht über einen langen Zeitraum, so wie es bei PMMA-Schichten beobachtet wurde [23]. In Abbildung 6.18 ist ein BisDMA/Cu-Schichtpaket vor (links) und nach der Verformung (rechts) durch einen Berkovich-Indent dargestellt. An den maximalen Krümmungspunkten der $\mathrm{Cu}$-Schichten entstehen Hohlräume. Hierbei konnte eine Schädigung durch das FIB ausgeschlossen werden. Es ist also eine Folge der Verformung. 


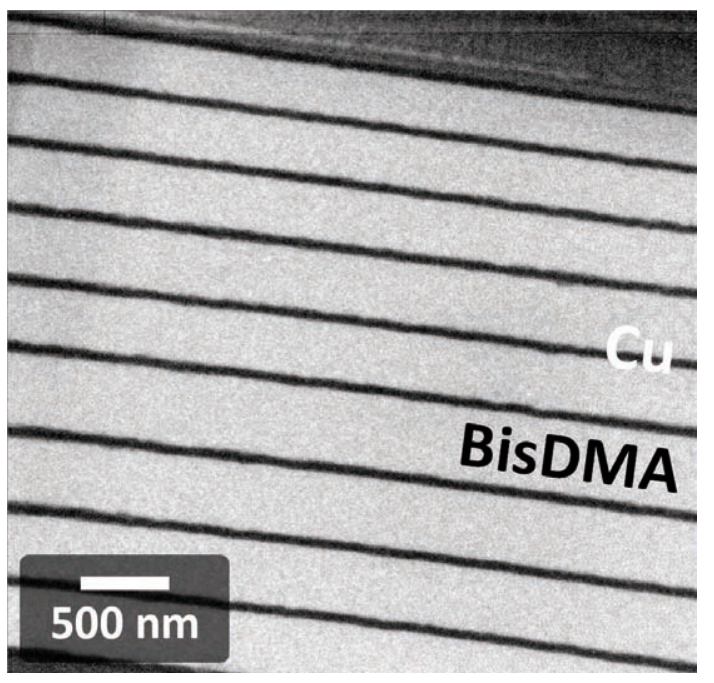

Abbildung 6.17: TEM-Aufnahme einer glatten periodischen BisDMA $(300 \mathrm{~nm}) / \mathrm{Cu}(30 \mathrm{~nm})-\mathrm{Multi}$ schicht. Die dunklen Schichten bestehen aus $\mathrm{Cu}$ und die hellen aus BisDMA. Alle Schichten sind glatt.
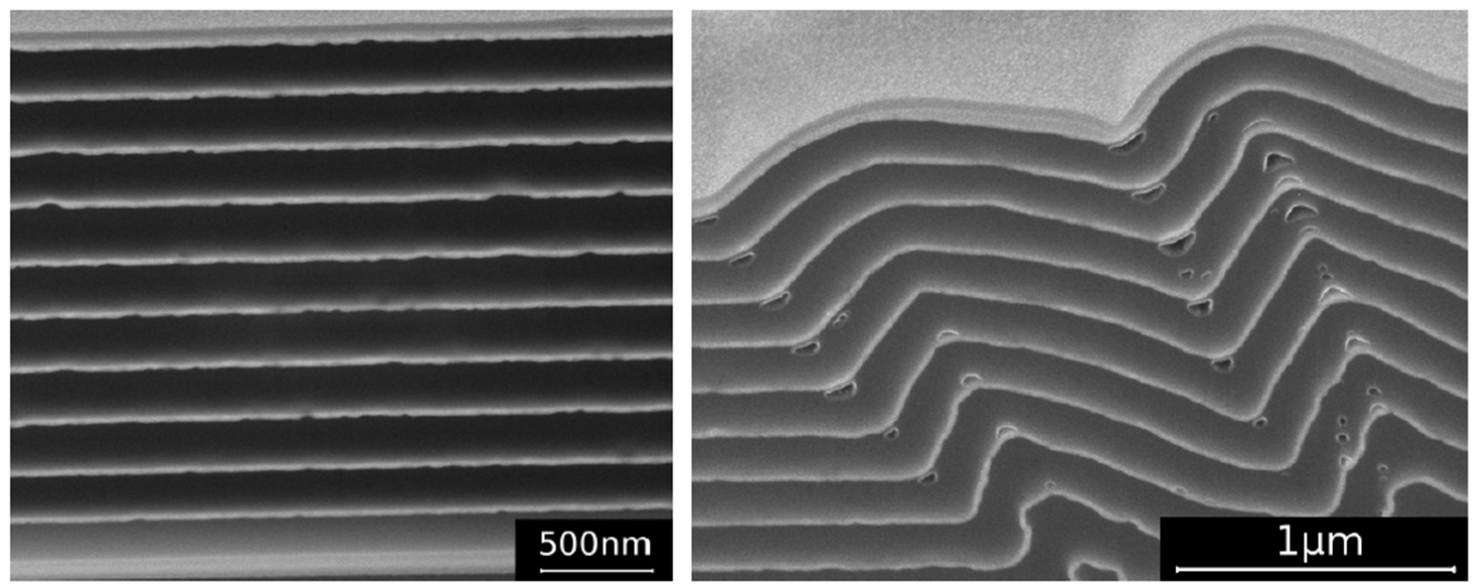

Abbildung 6.18: REM-Querschnittsaufnahmen eines periodischen BisDMA $(300 \mathrm{~nm}) / \mathrm{Cu}(30 \mathrm{~nm})$ Schichtpakets mit 10 Doppelschichten. Die linke Aufnahme zeigt das Schichtpaket nach der Herstellung. Die rechte Aufnahme zeigt das Schichtpaket nach der Verformung durch einen Berkovich-Indent (Bilder aus [60]).

Desweiteren wurden aus dem BisDMA/Cu-Schichtpaket mit Hilfe des FIBs Säulen präpariert, an denen anschließend Mikrodruckversuche stattfanden. In Abbildung 6.19 sind zwei REM-Aufnahmen von einer verformten Säule dargestellt. Es zeigt sich, dass durch die Verformung die Cu-Schichten aus dem Schichtpaket herausragen (siehe linkes Bild). Dies weist auf eine gute Haftung zum Substrat und zwischen den beiden Schichtkomponenten hin. Desweiteren ist in Abbildung 6.19 anhand der rechten REMAufnahme eine Hülle um die Säule zu erkennen. Diese Hülle entsteht durch eine 
Schädigung der BisDMA-Schichten aufgrund der Präparation mit dem FIB. Dieser Effekt wurde auch an Säulen aus reinem BisDMA beobachtet [60].
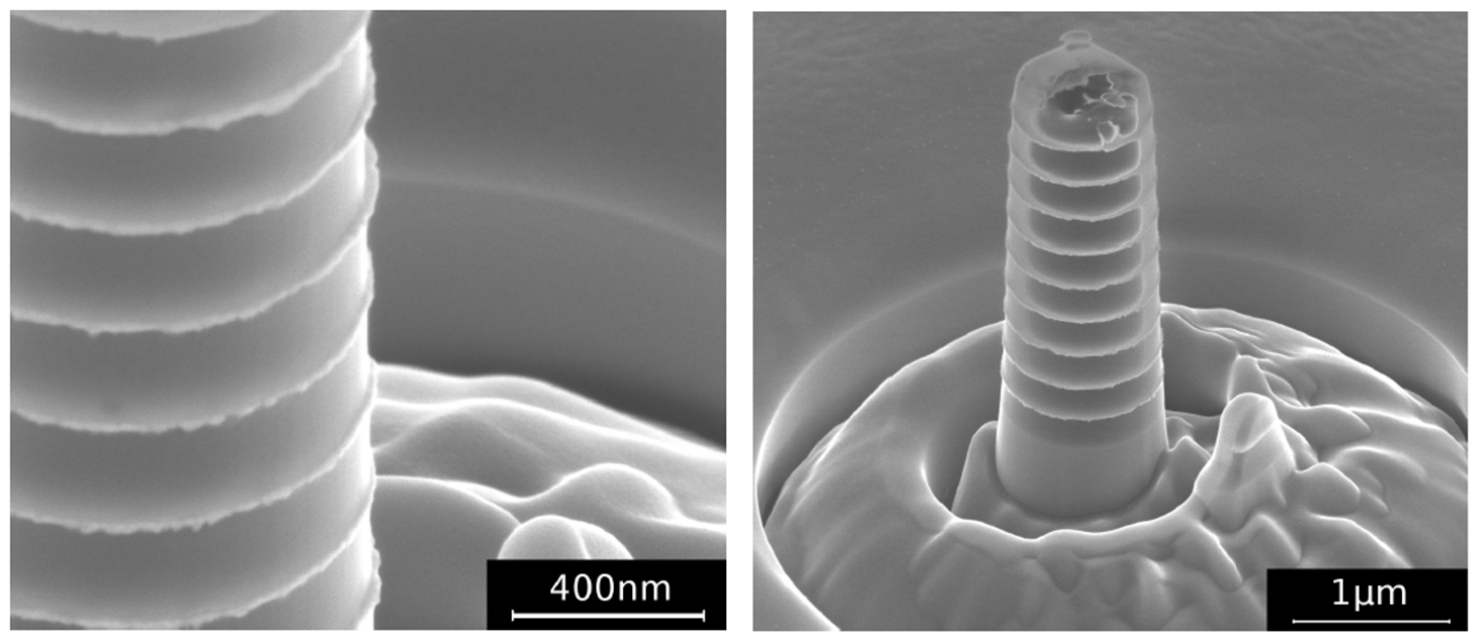

Abbildung 6.19: REM-Aufnahmen zweier Säulen, welche mittels FIB aus dem Schichtpaket präpariert wurden. Die Säulen wurden durch Mikrodruckversuche mechanisch verformt. Gute Haftungseigenschaften der Schichtkomponenten untereinander führen dazu, dass die Cu-Schichten aus dem Schichtpaket herausragen (Bilder aus [60]).

Insgesamt eignet sich das System BisDMA/Cu hervorragend für die Herstellung von glatten, periodischen Schichtpaketen. Bei der mechanischen Untersuchung der Schichtpakete durch Mikrodruckversuche von präparierten Säulen haben sich gute Haftungseigenschaften der beiden Schichtkomponenten untereinander und zum Substrat herausgestellt. Desweiteren ergaben die mechanischen Messungen, dass die BisDMASchichten wesentlich langzeitstabiler in Bezug auf Härte und E-Modul sind, als die PMMA-Schichten.

\subsubsection{PC/Cu-Multischichtsysteme}

Zur Herstellung glatter Polymer/Metall-Schichtsysteme eignet sich auch das PC, welches ebenso wie BisDMA einen E-Modul von $6 \mathrm{GPa}$ besitzt. Auch hier sollte durch die Variation der $\mathrm{Cu}$-Schichtdicke im PC/Cu-Schichtpaket untersucht werden, ob die PC-Schichten den Spannungen in den $\mathrm{Cu}$-Schichten widerstehen können, um glatte, periodische $\mathrm{PC} / \mathrm{Cu}-\mathrm{Multischichtsysteme} \mathrm{herstellen} \mathrm{zu} \mathrm{können.} \mathrm{Dazu} \mathrm{wurden}$ $\mathrm{Cu}$-Schichtdicken von $40 \mathrm{~nm}, 80 \mathrm{~nm}, 120 \mathrm{~nm}$ und $160 \mathrm{~nm}$ auf $300 \mathrm{~nm}$ dicke PC-Schichten deponiert. 


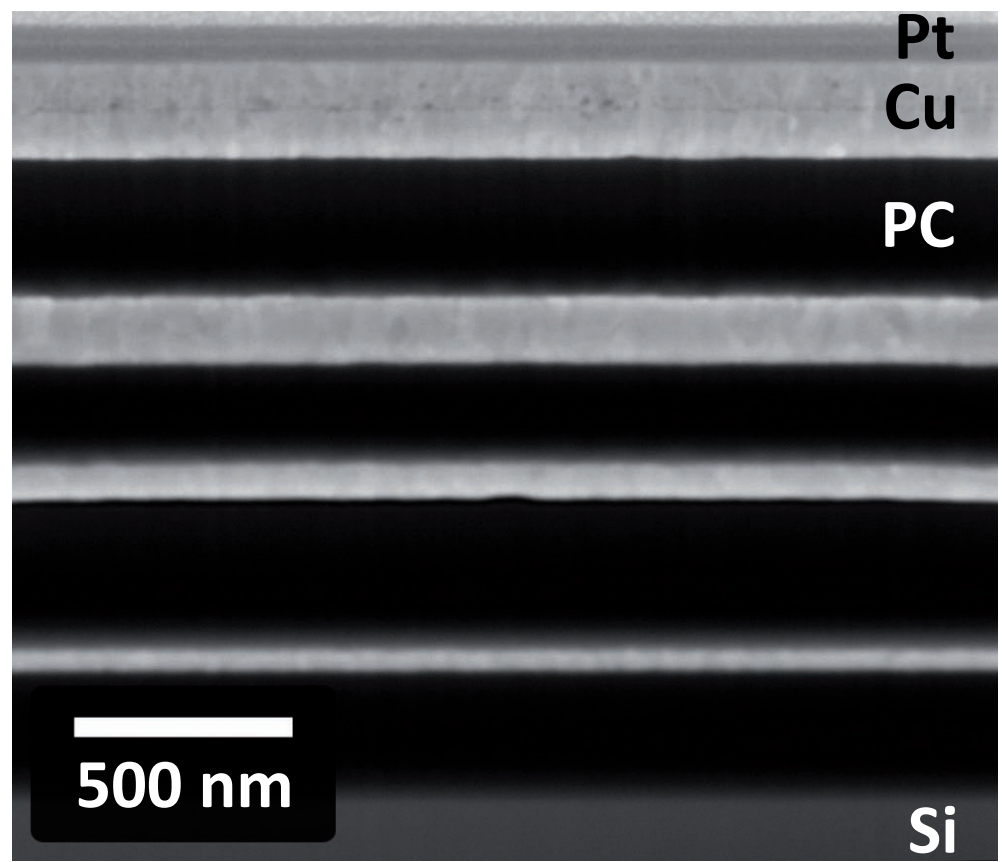

Abbildung 6.20: REM-Querschnittsaufnahme eines $\mathrm{PC}(300 \mathrm{~nm}) / \mathrm{Cu}$-Schichtpakets mit variierender $\mathrm{Cu}$-Schichtdicke von $40 \mathrm{~nm}, 80 \mathrm{~nm}, 120 \mathrm{~nm}$ und $160 \mathrm{~nm}$. Alle Schichten zeichnen sich durch eine sehr glatte Grenzfläche aus.

In Abbildung 6.20 ist das aperiodische Schichtpaket dargestellt. Hierbei zeigt sich, dass auch die dickste $\mathrm{Cu}$-Schicht glatt auf der PC-Schicht aufgewachsen ist. Dies bestätigt, dass der hohe Elastizitätsmodul von PC ausreicht, um den Spannungen in den $\mathrm{Cu}$-Schichten zu widerstehen. In Abbildung 6.21 ist anhand einer TEM-Aufnahme einer periodischen $\mathrm{PC}(25 \mathrm{~nm}) / \mathrm{Cu}(20 \mathrm{~nm})$-Multischicht dargestellt, dass alle Schichten sehr glatte Grenzflächen aufweisen.

Desweiteren hat sich ergeben, dass bei einem $\mathrm{PC} / \mathrm{Cu}$-Schichtpaket die $\mathrm{Cu}$ Schichtdicke immer größer als $10 \mathrm{~nm}$ sein muss, da sonst keine geschlossenen Schichten vorliegen. In Abbildung 6.22 sind zwei TEM-Bilder eines aperiodischen PC/CuSchichtpakets gezeigt. Hierbei ist in der linken TEM-Aufnahme der obere Teil des Schichtpakets mit der Pt-Deckschicht und in der rechten TEM-Aufnahme der untere Teil des Schichtpakets mit dem Substrat dargestellt. Es fällt auf, dass die ersten fünf CuSchichten (dunkle Linien, Abb. 6.22 rechts) noch nicht vollständig geschlossen sind, was sich an den feinen hellen Linien zwischen einigen $\mathrm{Cu}$-Körnern erkennen lässt. Wiederum zeigt die erste $\mathrm{Cu}$-Schicht, welche direkt auf dem Si-Substrat aufgewachsen ist, eine vollständig geschlossene Schicht, was auf den Substrateinfluss bzw. bessere Haftungseigenschaften zwischen $\mathrm{Cu}$ und $\mathrm{Si}$ anstelle von $\mathrm{Cu}$ auf $\mathrm{PC}$ zurückgeführt werden kann. Weiterhin lässt sich erkennen, dass sich die Cu-Körner durch die komplette Schichtdicke 


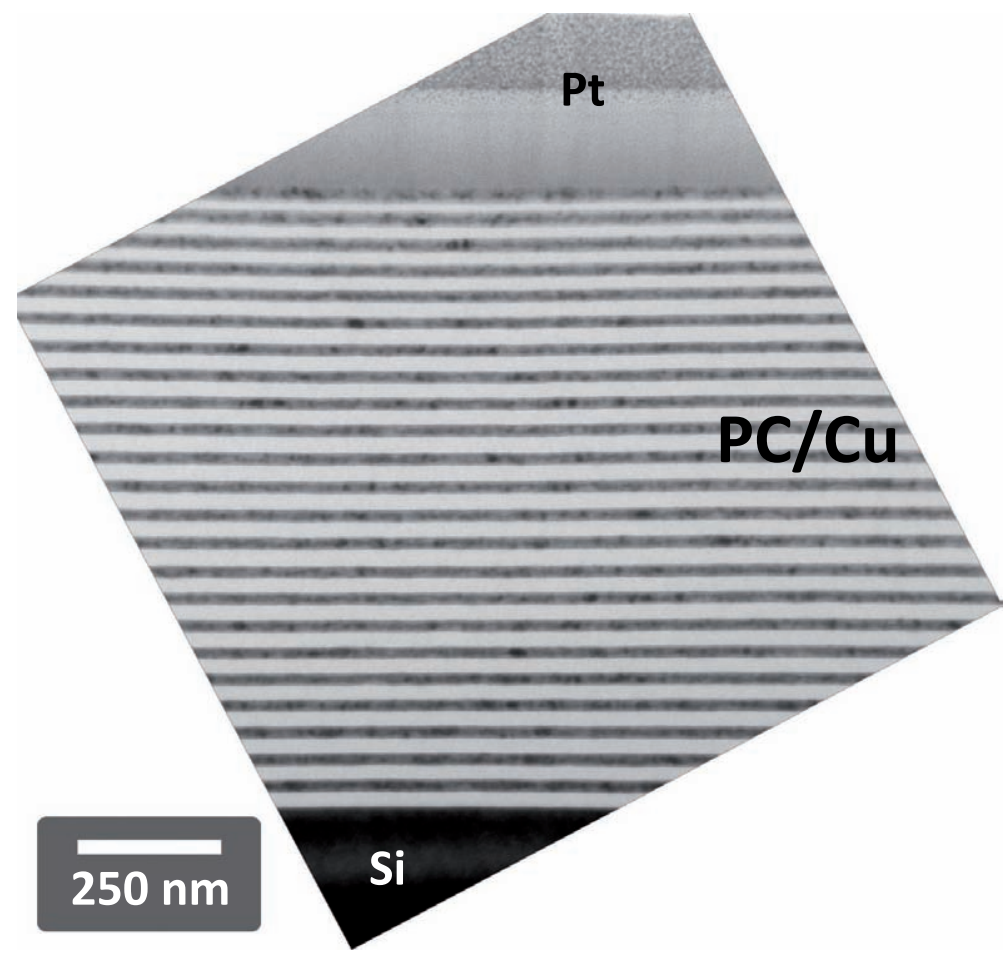

Abbildung 6.21: TEM-Aufnahme einer glatten, periodischen $\mathrm{PC}(25 \mathrm{~nm}) / \mathrm{Cu}(20 \mathrm{~nm})-\mathrm{Multischicht}$. Die dunklen Schichten bestehen aus $\mathrm{Cu}$ und die hellen aus PC.

erstrecken. Die amorphen PC-Schichten sind im Elektronenstrahl nahezu transparent. In der rechten Aufnahme weisen die $\mathrm{Cu}$-Schichten aufgrund eines $\mathrm{Cu}$-Droplets an dieser Stelle eine große Rauigkeit auf. Jedoch ist hier zu erkennen, dass diese Rauigkeiten sich bis zur obersten Deckschicht fast selbstständig wieder ausglätten, was der hohen Ionenenergie bei der gepulsten Laserdeposition geschuldet ist [91]. Insgesamt lässt sich ein sehr scharfer Übergang zwischen den beiden Materialien erkennen.

Eine Vielzahl an glatten, periodischen $\mathrm{PC} / \mathrm{Cu}$-Schichtpaketen mit variablen PC- und $\mathrm{Cu}$-Schichtdicken ist in Zusammenarbeit mit Inga Knorr und Julia Oelker in Bezug auf die mechanischen Eigenschaften der Schichtpakete untersucht worden. Hierbei wurden Berkovich-Indents und Mikrodruckversuchen an den Proben durchgeführt. Insbesondere für die Säulenpräparation im FIB hat sich gezeigt, dass das PC im Vergleich zu den anderen Polymeren (PMMA, BisDMA) am besten handzuhaben ist [74]. In Abbildung 6.23 sind zwei REM-Aufnahmen von einer im FIB präparierten Säule aus einem PC/Cu-Schichtpaket dargestellt. Im linken Bild ist die Säule vor dem Mikrodruckversuch dargestellt und im rechten Bild nach der Verformung. Hierbei zeigt sich, dass sich die Cu-Schichten stärker verformen und aus der Säule herausquillen. Dies 

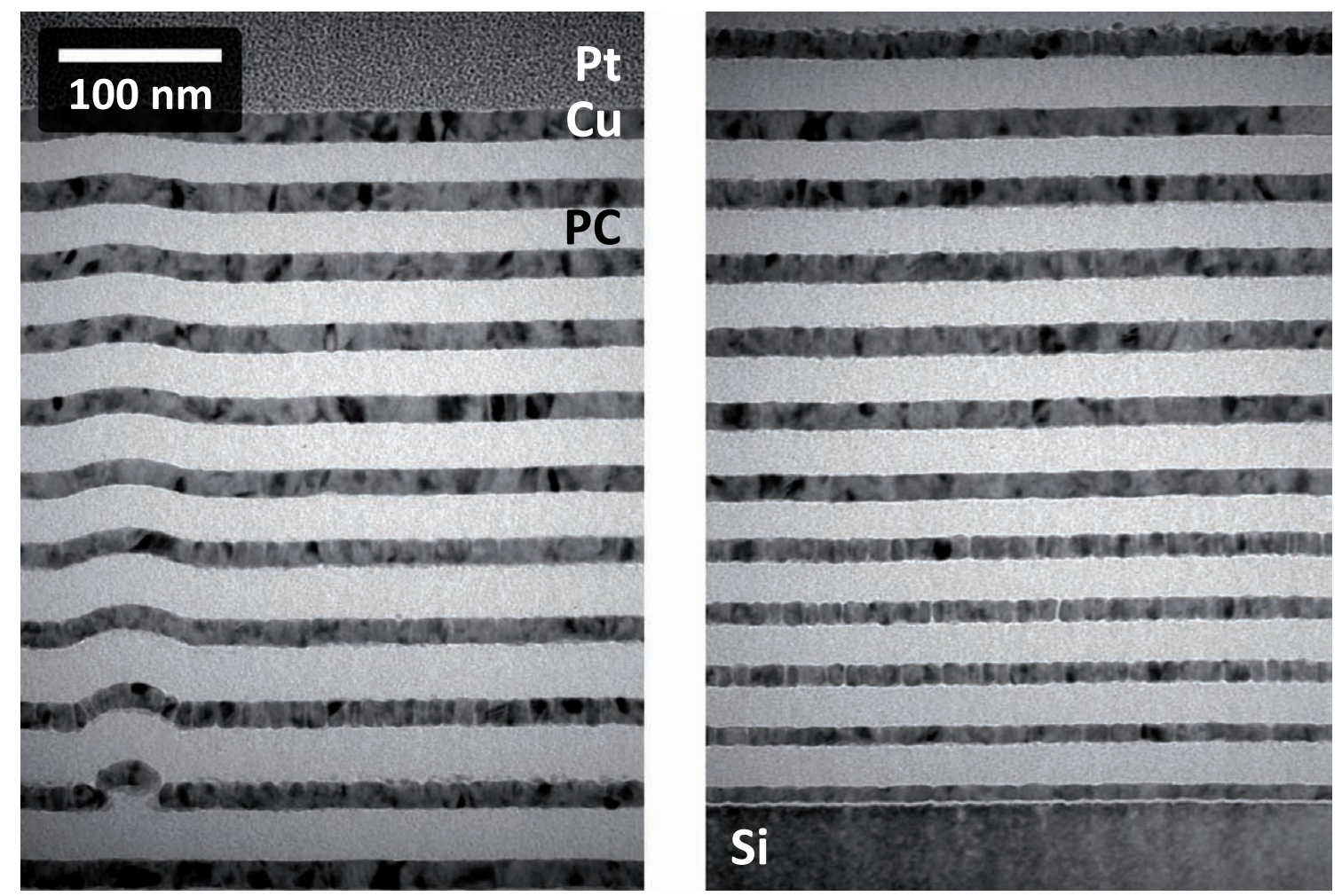

Abbildung 6.22: TEM-Aufnahmen einer PC/Cu-Multischicht. Im linken Bild, welche den oberen Teil des Schichtpakets mit der Pt-Deckschicht zeigt, ist ein $\mathrm{Cu}$-Droplet zu erkennen. Die durch den Droplet verursachten Rauigkeiten glätten sich selbstständig bis zur obersten Deckschicht nahezu vollständig aus. Im rechten Bild, welches den unteren Teil des Schichtpakets mit dem Si-Substat darstellt, sind die ersten fünf $\mathrm{Cu}-\mathrm{Schich}$ ten noch nicht vollständig geschlossen.

weist insgesamt wie bereits im BisDMA/Cu-System beobachtet auf eine gute Haftung zwischen den beiden Komponenten hin.

Desweiteren wurden die Proben auch anhand von Berkovich-Indents untersucht. In Abbildung 6.24 sind mehrere REM-Querschnittsaufnahmen eines solchen verformten $\mathrm{PC}(100 \mathrm{~nm}) / \mathrm{Cu}(50 \mathrm{~nm})$-Schichtpakets gezeigt. In den Bildern (d, f) drang der Berkovich-Indent $200 \mathrm{~nm}$ tief in das Material ein, und in den Bildern (e, g) bis zu $1 \mu \mathrm{m}$. Es zeigt sich, dass es zur Ausbildung von interlayer-Scherbändern kommt. Hierbei ist das PC stark gestaucht worden, so dass das Schichtmaterial nach aussen, weg vom Indent, geschoben wurde. Desweiteren konnte keine Delamination weder an den Grenzflächen noch am Substrat beobachtet werden. Auch dies weist auf eine sehr gute Haftung zwischen den beiden Schichtkomponenten und zum Substrat hin.

Zusammenfassend konnte gezeigt werden, dass sich das $\mathrm{PC} / \mathrm{Cu}$-Schichtsystem sehr gut für die Herstellung glatter, periodischer Multischichten eignet. Hierbei haben die 


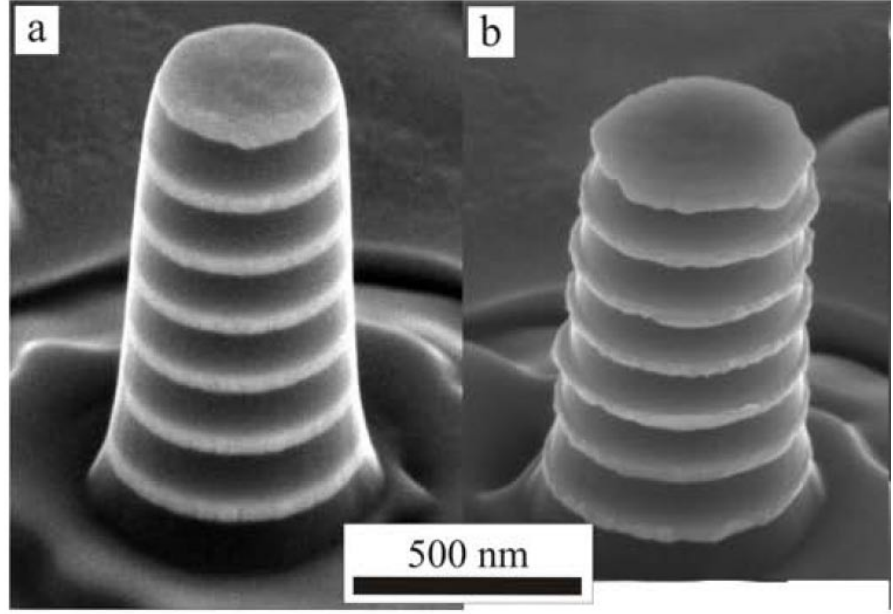

Abbildung 6.23: REM-Aufnahmen von im FIB präparierten Säulen aus einem PC/Cu-Schichtpaket. Im linken Bild ist die Säule vor dem Mikrodruckversuch dargestellt und im rechten Bild nach der Verformung (Daten aus [74]).

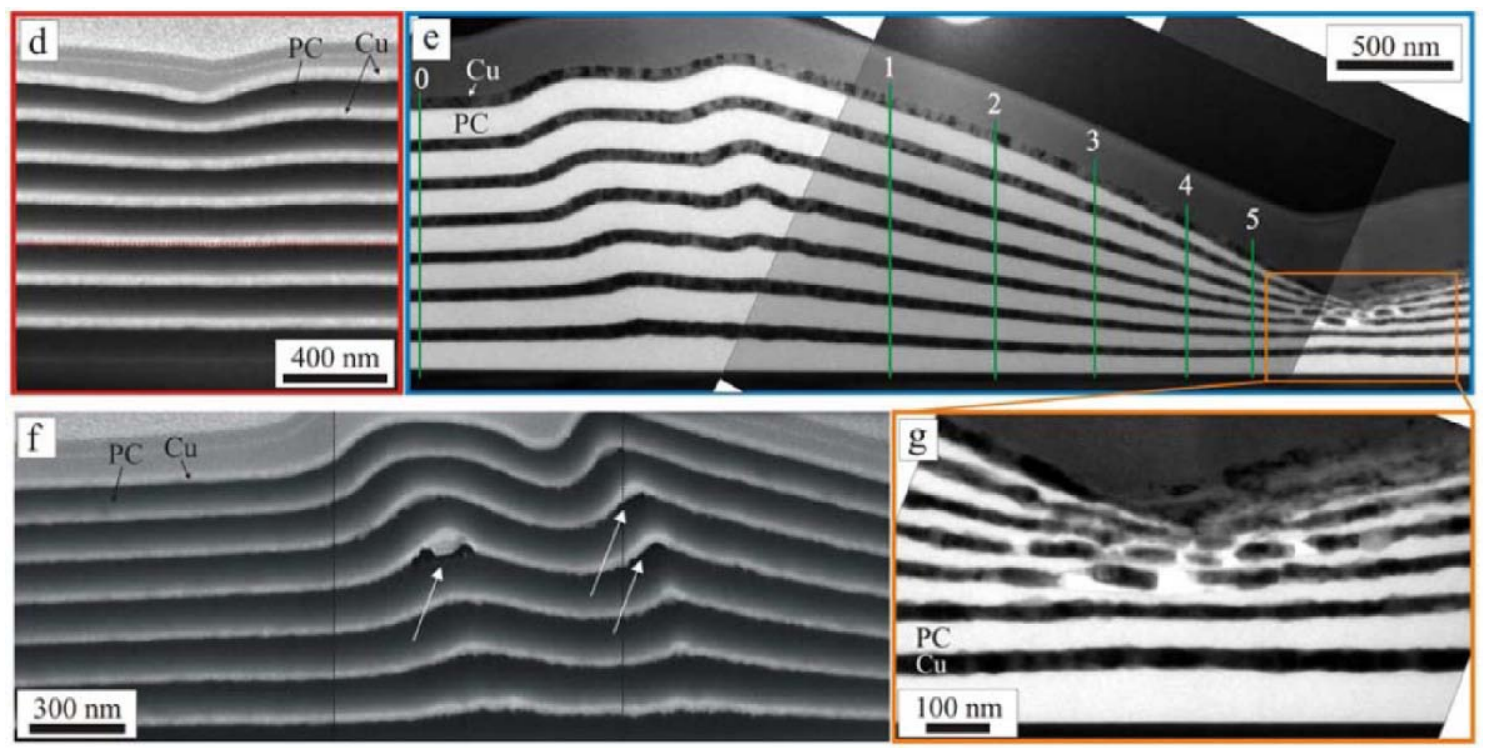

Abbildung 6.24: REM-Querschnittsaufnahmen eines $\mathrm{PC}(100 \mathrm{~nm}) / \mathrm{Cu}(50 \mathrm{~nm})$-Schichtpaketen, welches durch einen Berkovich-Indent verformt wurde. Hierbei ist in Bild (d, f) der Berkovich-Indent $200 \mathrm{~nm}$ tief ins Material und in Bild (e,g) $1 \mu \mathrm{m}$ tief gedrückt worden (Daten aus [74]).

mechanischen Untersuchungen gezeigt, dass eine sehr gute Haftung sowohl innerhalb des Schichtpakets als auch zum Substrat vorliegt. Die Schichtsysteme bestehend aus einer amorphen und einer kristallinen Komponente mit einer scharfen Grenzfläche sind insbesondere für neue Fragestellungen von Interesse. Im Sonderforschungsbereich 1073 soll der Effekt des „phonon blocking“ an diesen Polymer/Metall-Schichtpaketen untersucht werden. 



\section{Zusammenfassung}

In dieser Arbeit wurde die spannungsinduzierte Wellenbildung in laserdeponierten Polymer/Metall-Schichtsystemen untersucht. Hierbei haben sowohl die Polymerschichten als auch die Metallschichten bedingt durch die verwendete Herstellungsmethode besondere Eigenschaften. Aufgrund der Laserdeposition sind die PMMA-Schichten in ihrer Bindungsstruktur modifiziert und weisen eine verkürzte Kettenlänge auf. Daraus resultiert ein sehr geringer Elastizitätsmodul. Desweiteren wachsen die laserdeponierten Metallschichten aufgrund der hohen kinetischen Energien der deponierten Teilchen (ca. $100 \mathrm{eV}$ ) stark verspannt auf. Die aus der Verdichtung des Materials resultierenden Spannungen in den Metallschichten können dabei nur durch die Ausbildung von Wellen an der Oberfläche kompensiert werden, da die weiche PMMA-Unterlage auf dem SiSubstrat sehr gut haftet.

Im ersten Teil der Arbeit konnte gezeigt werden, dass das verwendete PMMA, aufgrund fehlender Absorptionszentren in Form von Doppelbindungen im Targetmaterial, zunächst ein schwacher UV-Absorber ist. Während der Inkubation des Targetmaterials, welche durch NEXAFS-Messungen detailliert untersucht wurde, sind durch eine ca. 2000 Laserpulse andauernde Modifikation $\mathrm{C}=\mathrm{C}$-Doppelbindungen im Target entstanden, die den Absorptionsgrad der UV-Strahlung drastisch erhöhen. Diese Modifikation führt dazu, dass die laserdeponierten PMMA-Schichten um bis zu drei Größenordnungen verkürzte Kettenlängen besitzen. Damit geht auch eine Absenkung der Glasübergangstemperatur als auch eine geringere Viskosität der Schichten einher. Die Viskosität der Schichten konnte auf einen Wert von 180 Pas abgeschätzt werden, welche um zehn Größenordnungen geringer ist als die des Ausgangsmaterials. Desweiteren ist anhand von AFM-Indentermessungen ein Elastizitätsmodul für die PMMA-Schichten im unteren MPa-Bereich abgeschätzt worden. Weiterhin zeigen die PMMA-Schichten ein viskoelastisches Verhalten, wobei sich die Schichten auf kurzen Zeitskalen wie ein elastischer Festkörper und auf langen Zeitskalen (> 1s) wie eine viskose Flüssigkeit verhalten. Neben PMMA wurden auch die Polymere BisDMA und PC untersucht, welche im Targetmaterial eine Vielzahl an $\mathrm{C}=\mathrm{C}$ - 
Doppelbindungen vorweisen und somit eine vergleichsweise höhere UV-Absorption als das PMMA aufweisen. Dieser veränderte Ablationsprozess führt auch dazu, dass die Polymerschichten aus BisDMA und PC im Vergleich zu den PMMA-Schichten einen deutlich höheren Elastizitätsmodul von $6 \mathrm{GPa}$ besitzen. Es ließen sich mit allen Polymeren glatte Schichten herstellen. Die Kombination von PMMA mit BisDMA bzw. PC ermöglichte durch eine Vernetzungsanregung die Kettenlänge und somit auch die mechanischen Eigenschaften (Härte, E-Modul) gezielt zu steigern und einzustellen.

Weiterhin wurde systematisch untersucht, wie sowohl die PMMA- als auch die CuSchichtdicke die Ausbildung der Wellen beeinflusst, und dass es dadurch möglich ist, die Wellenlänge gezielt über mehrere Mikrometer einzustellen. Dabei hat sich gezeigt, dass das Auftreten von Wellen ein guter Indikator ist, um die vorliegenden Spannungen in den $\mathrm{Cu}$-Schichten $\mathrm{zu}$ verfolgen und $\mathrm{zu}$ verstehen. Insbesondere konnte in den einzelnen Wachstumsstadien der $\mathrm{Cu}$-Schichten, beginnend mit isolierten $\mathrm{Cu}-\mathrm{Clustern}$, über Koaleszenz bis hin zu geschlossenen Schichten, ein Wechsel von Druck- zu Zug- und wiederum zu Druck-Spannungen anhand der aus den Wellen resultierenden Oberflächenrauigkeit nachgewiesen werden. Die Mechanismen, die für diesen Spannungswechsel sorgen, sind in den Frühstadien des Wachstums mit isolierten Clustern der Effekt der Gitterparameteraufweitung, welcher ca. $10 \%$ beträgt. Durch eine Abschätzung konnte nachgewiesen werden, dass das Amplituden-Wellenlängenverhältnis, bei dem die resultierenden Wellen des Polymer/Metall-Systems als sinusförmig angenommen wurden, genau diesen $10 \%$ entspricht. Zusätzlich werden Druckspannungen in den $\mathrm{Cu}$-Schichten durch Ionenimplantation hervorgerufen, welche zu einer Verdichtung des Materials („shot peening“) führen. Der Wechsel zu Zugspannungen resultiert aus Koaleszenzprozessen (,island zipping“"). Mit Ausbildung geschlossener Schichten überwiegt wieder der Einfluss des Verdichtens von Schichtmaterial, wodurch der Wechsel zu Druckspannungen erklärt wird. Die verschiedenen Spannungskomponenten haben demnach großen Einfluss auf die Wellenbildung und machen es sogar möglich, die Wellen beliebig in ihrer Größe einzustellen. So sind im Spannungsgleichgewicht von Zug- und Druckspannungen bei einer Cu-Schichtdicke von ca. $5 \mathrm{~nm}$ keine Wellen mehr vorhanden, wohingegen extrem große Wellen mit Wellenlängen von mehreren Mikrometern vor allem im Wachstumsstadium der geschlossenen Schichten auftreten.

Wie sich bei der systematischen Untersuchung der Wellenlänge in Abhängigkeit der PMMA-Schichtdicke gezeigt hat, existiert ein Einfluss des Si-Substrates, der durch Variation der PMMA-Schichtdicke eine gezielte Einstellung der Wellenlänge über mehrere Mikrometer ermöglicht. Eine kritische PMMA-Schichtdicke von $20 \mu \mathrm{m}$ konnte 
bestimmt werden, bei der der Substrateinfluss seinen Einfluss verliert. Diese sehr große kritische Schichtdicke konnte durch den Wechsel zu einem PMMA-Substrat (mit geringerem E-Modul als vergleichsweise eines Si-Substrates) auf $6 \mu \mathrm{m}$ deutlich verringert werden. Für derartig hergestellte Schichten ohne Substrateinfluss konnte erstmalig mit Hilfe der SIEBIMM-Methode der E-Modul der laserdeponierten PMMASchicht von lediglich $3 \mathrm{MPa}$ bestimmt werden. Dieser Wert konnte durch AFMIndentermessungen und durch Vergleiche mit Elastizitätsmodul-Werten für Polymere aus der Literatur bestätigt werden.

Mit der Kenntnis des E-Moduls der PMMA-Schicht war es zudem möglich unter Vermeidung des Substrateinflusses die E-Moduln verschiedenster Materialklassen, wie Metalle, Oxide und Polymere zu bestimmen, da alle Systeme aus PMMA mit der jeweiligen zu testenden Schicht Wellenbildung zeigen. Dadurch zeichnet sich diese Messmethode zur Bestimmung des Elastizitätsmoduls durch ihre Vielfältigkeit aus.

Eine weitere Möglichkeit die Wellenlänge in den Polymer/Metall-Systemen zu beeinflussen wurde durch die Schichtherstellung in einer Ar-Gasatmosphäre realisiert. Hierbei konnten gezielt die einzelnen Spannungszustände in den Metallschichten in Abhängigkeit vom Ar-Partialdruck eingestellt werden. Insbesondere in dem Druckbereich um 0,1 mbar war es dadurch möglich, nahezu spannungsfreie Cu-Schichten herzustellen mit einer sehr geringen Rauigkeit von $29 \mathrm{~nm}$. Allerdings hat sich gezeigt, dass dieser Druckbereich, bei dem Zug- und Druckspannungen im Gleichgewicht sind, sehr klein ist, so dass es sich als experimentell schwierig erweist über diese Möglichkeit glatte Polymer/Metall-Schichtsysteme herzustellen.

Neben einem gezielten Einstellen der Wellenlänge ist überdies der Versuch unternommen worden, die Wellen gezielt anzuordnen. Dazu wurden in dieser Arbeit verschiedenste Möglichkeiten aufgezeigt. Einerseits wurden durch die Deposition der $\mathrm{Cu}$-Schicht unter einem Winkel die Wellen durch einen selbstorganisierten Prozess über eine Reichweite von $1 \mathrm{~cm}$ angeordnet. Andererseits konnte im System PMMA/Pd durch einfaches Ritzen der PMMA-Schicht eine Anordnung über eine Reichweite von $500 \mu \mathrm{m}$ erzielt werden.

Im zweiten Teil der Arbeit wurden Strategien zum Erreichen glatter, periodischer Polymer/Metall-Schichtsysteme untersucht. Hierbei wurde ein Verfahren zur Steigerung des E-Moduls der PMMA-Schicht gefunden. Zum einen konnte der E-Modul der PMMA-Schichten durch eine UV-Bestrahlung der PMMA-Schichten gezielt gesteigert und eingestellt werden. Hierbei wurden die Ketten durch die UV-Strahlung zum 
Quervernetzen angeregt. Dadurch ist es gelungen ein PMMA/Cu-Schichtpaket mit teilweise glatten $\mathrm{Cu}$-Schichten herzustellen. Auch durch eine Temperaturbehandlung der PMMA-Schichten konnte der E-Modul-Wert gezielt gesteigert und eingestellt werden, wodurch die Herstellung glatter $\mathrm{Cu}$-Schichten möglich wurde. Zuletzt konnte gezeigt werden, dass sich der E-Modul der PMMA-Schichten durch das „Dazulegieren“ von PC gezielt steigern und einstellen lässt. Hierbei konnte ein kritischer Wert von 1,4\% für den Anteil an PC gefunden werden, was einem E-Modul von $1 \mathrm{GPa}$ der PMMA/PC-Mischschicht entspricht, welcher ausreicht glatte $\mathrm{Cu}$-Schichten auf den PMMA/PC-Mischschichten zu deponieren. Die dabei zugrundeliegende kritische Spannung zwischen den beiden Komponenten konnte auf einen Wert von 2, 6 GPa für einen E-Modul von $1 \mathrm{GPa}$ bestimmt werden.

Für die Herstellung von glatten, periodischen Polymer/Metall-Schichtpaketen eignet sich das PMMA aufgrund seines geringen E-Moduls (3 MPa) nur bedingt. Im Gegensatz dazu lassen sich mit den Polymeren BisDMA und PC glatte, periodische Schichtpakete mit beliebigen Polymer- und Metallschichtdicken herstellen. Diese Schichtpakete zeichnen sich durch eine sehr gute Haftung sowohl der Grenzflächen als auch zum Substrat aus, wie anhand von Untersuchungen der mechanischen Eigenschaften dieser Schichtpakete gezeigt werden konnte.

Insgesamt konnte in dieser Arbeit ein grundlegendes Verständnis der spannungsinduzierten Wellenbildung in laserdeponierten Polymer/Metall-Schichtsystemen erarbeitet werden. Die Ausbildung der Wellen konnte für die Etablierung einer neuen Messmethode angewendet werden, mit der es erstmals möglich war, den Elastiziätsmodul von laserdeponierten PMMA-Schichten zu bestimmen. Weiterhin wurden Strategien zum Erreichen von glatten, periodischen Polymer/Metall-Schichtsystemen entwickelt und diese auch hergestellt. 


\section{Literaturverzeichnis}

[1] Gebauer V.: Untersuchung zum Einfluss der altersbedingten Veränderungen der Haut auf die Effektivität von Sonnenschutzmitteln. Dissertation Medizinische Fakultät Charite-Universitätsmedizin Berlin (2012)

[2] Genzer J., Groenewold J.: Soft matter with hard skin: From skin wrinkles to templating and material characterization. Soft matter 2 (4) (2006), S. 310-323

[3] Thomas K., Herminghaus S., Porada H., Goehring L.: Formation of Kinneyia via shear-induced instabilities in microbial mats. Phil Trans R Soc A 371 (2013), S. 20120362

[4] Cunningham R.L., Haymann P., Lecomte C., Moore W.J., Trillat J.J.: Etching of surfaces with 8-Kev Argon Ions. Journal of Applied Physics 31 (1960), S. 839-842

[5] Sigmund P.: A mechanism of surface micro-roughening by ion bombardment. Journal of material science 8 (1973), S. 1545-1553

[6] Mayr S. G., Averback R.S.: Surface Smoothing of Rough Amorphous Films by Irradiation-Induced Viscous Flow. Physical Review Letters 87 (2001), S. 19

[7] Bradley R.M., Harper J.M.E.: Theory of ripple topography induced by ion bombardement. Journal of Vacuum Science and Technology A 6 (1988), S. 2390

[8] Habenicht S., Bolse W., Lieb K. P.: Nanometer ripple formation and self-affine roughening of ion-beam-eroded graphite surfaces. Physical Review B 60 (1999), S. 4

[9] Martin G.C., Su T.T., Loh I.H., Balizer E., Kowel S. T., Kornreich P.: The metallization of silicone polymers in the rubbery and the glassy state. Journal of Applied Physics 53 (1982), S. 797

[10] Bowden N., Brittain S., Evans A. G., Hutchinson J. W., Whitesides G. M.: Spontaneous formation of ordered structures in thin films of metals supported on an elastomeric polymer. Nature 393 (1998), S. 146

[11] Bowden N., Huck W. T. S., Paul K. E., Whitesides G. M.: The controlled formation of ordered, sinusoidal structures by plasma oxidation of an elastomeric polymer. Applied physics letters 75 (1999), S. 17 
[12] Huck W.T.S., Bowden N., Onck P., Pardoen T., Hutchinson J. W., Whitesides G. M.: Ordering of spontaneously formed buckles on planar surfaces. Langmuir 16 (2000), S. $3497-3501$

[13] Stafford C. M., Harrison C., Beers K. L., Karim A., Amis E. J., Vanlandigham M. R., Kim H., Volksen W., Miller R. D., Simonyi E. E.: A buckling-based metrology for measuring the elastic moduli of polymeric thin films. nature materials 3 (2004), S. 545

[14] Ohzono T., Shimomura M.: Ordering of microwrinkle patterns by compressive strain. Physical Review B 69 (2004), S. 1332202

[15] Efimenko K., Rackaitis M., Manias E., Vaziri A., Mahadevan L., Genzer J.: Nested self-similar wrinkling patterns in skins. Nature materials 4 (2005), S. 293-297

[16] Chung J. Y., Nolte A. J., Stafford C. M.: Surface Wrinkling: A Versatile Platform for Measuring Thin-Film Properties. Adv. Mater. 23 (2011), S. 349-368

[17] Schweikart A., Fery A.: Controlled wrinkling as a novel method for the fabrication of patterned surfaces. Microchim Acta 165 (2009), S. 249-263

[18] Lu C., Möhwald H., Fery A.: A lithography-free method for directed colloidal crystal assembly based on wrinkling. Soft Matter 3 (2007), S. 1530-1536

[19] Sabbah A., Vandeparre H., Braub F., Dammana P.: Self-Cleaning Surfaces Prepared By Microstructuring System. Physics Procedia 21 (2011), S. 193-197

[20] Vandeparre H., Desbief S., Lazzaroni R., Gay C., Damman P.: Confined wrinkling: impact on pattern morphology and periodicity. Soft Matter 7 (2011), S. 6878

[21] Chrisey D.B., Hubler G. K.: Pulsed Laser Deposition of Thin Films. John Wiley \& Sons, Inc. (1994)

[22] Eason R.: Pulsed Laser Deposition of Thin Films. Wiley, New Jersey (2007)

[23] FuCHS B. L.: Charakterisierung und Modifizierung glatter laserdeponierter Poly(methyl methacrylat)-Schichten. Dissertation Institut für Materialphysik, Universität Göttingen (2010)

[24] FÄHLER S.: Der Einfluss der kinetischen Energie der Ionen auf das Wachstum laserdeponierter metallischer Schichten und Schichtpakete: Untersuchungen mit Flugzeitmessungen und RHEED/THEED. Dissertation Institut für Materialphysik, Universität Göttingen (1998)

[25] Faehler S., Krebs H.-U.: Calculations and experiments of material removal and kinetic energy during pulsed laser ablation of metals. Appl. Surf. Sci. 96 (1996), S. S.61 
[26] Krebs H.U.: Characteristic properties of laser-deposited metallic systems. International Journal of Non-Equilibrium Processing 10 (1997), S. 3-24

[27] Lunney J. G.: Pulsed laser deposition of metal and metal multilayer films. Applied Surface Science 86 (1995), S. 79-85

[28] NiR D.: Stress relief forms of diamond-like carbon thin films under internal compressive stress. Thin solid films 112 (1984), S. 41-49

[29] Iyer S. B., Harshavardhan K. S., Kumar V.: Buckling patterns in diamond-like carbon films. Thin solid films 256 (1995), S. 94-100

[30] Mang H., Hofstetter G.: Festigkeitslehre. Springer Wien, New York (2004)

[31] Schmidt: 6. Balken und Rahmen. Vorlesungsskript URL http://www4.iam.rwthaachen.de/Lehre/SS-09/Mechanik-Prof-Schmidt/Festigkeitslehre-Schmidt-66.pdf (2009)

[32] Landau L. D., Lifschitz E. M.: Band VII Elastizitätstheorie. Akademie Verlag, Berlin (1966)

[33] Allen H. G.: Analysis and Design of Structural Sandwich Panels. Pergamon, New York (1969)

[34] Chan E. P., Page K. A., Im S. H., Patton D. L., Huang R., Stafford C. M.: Viscoelastic properties of confined polymer films measured via thermal wrinkling. Soft matter 5 (2009), S. 4638-4641

[35] Volynskit A. L., Bazhenov S., Lebedeva O. V., Bakeev N. F.: Mechanical buckling instability of thin coatings deposited on soft polymer substrates. Journal of Materials Science 35 (2000), S. 547-554

[36] Biот M. A.: Folding instability of a layered viscoelastic medium under compression. Proc. R. Soc. A 242 (1957), S. 444-454

[37] Biот M. A.: Surface instability of rubber in compression. Applied Scientific Research A 12 (1963), S. 168-182

[38] Faehler S., Kahl S., Weisheit M., Sturm K., Krebs H.-U.: The interface of laser deposited Cu/Ag multilayers: evidence of the „subsurface growth mode“ during pulsed laser deposition. Appl. Surf. Sci. 154-155 (2000), S. S.419

[39] Kelly R., Cuomo J. J., Leary P. A., Rothenberg J. E., Braren B. E., Aliotta C. F.: Laser sputtering Part I. On the existence of rapid laser sputtering at $193 \mathrm{~nm}$. Nuclear Instruments and Methods in Physics Research B 9 (1985), S. 329-340

[40] Singh R. K., NARAyan J.: Pulsed-laser evaporation technique for deposition of thin films: Physics and theoretical model. Physical Review B 41 (1990), S. 8843-8859 
[41] Binnig G., Quate C. F., Gerber Ch.: Atomic Force Microscope. Phys. Rev. Lett. 56 (1986), S. 930

[42] Hafner B.: Scanning Electron Microscopy Primer. Characterization Facility, University of Minnesota-Twin Cities (2007), S. S.1

[43] Hafner B.: Introductory Transmission Electron Microscopy Primer. Characterization Facility, University of Minnesota-Twin Cities (2008), S. S.1

[44] Volkert C. A., Minor A. M.: Focused ion beam microscopy and micromachining. MRS Bulletin 32 (2007), S. 389

[45] Grossmann Peter: Optimierung und Charakterisierung einer XUV-Laborröntgenquelle für die zeitaufgelöste Röntgenabsorptionsspektroskopie. Dissertation Technische Universität Clausthal (2012)

[46] Lazare S., Lopez J., F. Weisbuch: High-aspect-ratio microdrilling in polymeric materials with intense KrF laser radiation. Applied Physics A-Materials Science \& Processing 69 (1999), S. S1

[47] Dyer P. E.: Excimer laser polymer ablation: twenty years on. Applied Physics A 77 (2003), S. 167

[48] Chrisey D.B., Piqu’e A., A. McGill R., Horwitz J. S., Ringeisen B. R., Bubb D. M., Wu P. K.: Laser Deposition of Polymer and Biomaterial Films. Chem. Rev. 103 (2003), S. 553

[49] Lippert T.: Interaction of Photons with Polymers: From Surface Modification to Ablation. Plasma Process. Polym. 2 (2005), S. 525

[50] Lippert T., Dickinson J. T.: Chemical and Spectroscopic Aspects of Polymer Ablation: Special Features and Novel Directions. Chem. Rev. 103 (2003), S. 453

[51] Lippert T., WebB R. L., Langford S. C., Dickinson J. T.: Dopant induced ablation of poly(methyl methacrylate) at 308 nm. J. Appl. Phys. 85 (1999), S. 1838

[52] SüSkE E.: Charakteristische Eigenschaften laserdeponierter Poly(methyl methacrylat)Filme. Dissertation Institut für Materialphysik, Universität Göttingen (2005)

[53] Dhez O., Ade H., Urquhart S. G.: Calibrated NEXAFS spectra of some common polymers. Journal of Electron Spectroscopy and Related Phenomena 128 (2003), S. $85-96$

[54] Schlenkrich Felix: Beeinflussung des Wachstums von Metall auf Polymer durch die gepulste Laserdeposition. Dissertation Institut für Materialphysik, Universität Göttingen (2014) 
[55] Fuchs B., Schlenkrich F., Seyffarth S., Meschede A., Rotzoll R., Vana P., Grossmann P., Mann K., Krebs H.-U.: Hardening of smooth pulsed laser deposited PMMA films by heating. Applied Physics A-Materials Science \& Processing 98 (2010), S. $711-715$

[56] K. O'Driscoll, A. Sanayei R.: Chain-length dependence of the glass transition temperature. Macromolecules 24 (1991), S. 4479-4480

[57] Sperling L.H.: Introduction to physical polymer science. John Wiley and Sons, Inc. second edition (1992)

[58] Krevelen D.W. V.: Properties of Polymers. Elsevier (1990)

[59] Fuch K., Friedrich Chr., Weese J.: Viscoelastic Properties of Narrow-Distribution Poly(methylmethacrylates). Macromolecules 29 (1996), S. 5893-5901

[60] Wuttke T.: Mechanische Eigenschaften von Kupfer-BisDMA-Multischichten. Bachelorarbeit Institut für Materialphysik, Universität Göttingen (2010)

[61] Domke J., Radmacher M.: Measuring the Elastic Properties of Thin Polymer Films with the Atomic Force Microscope. Langmuir 14 (1998), S. 3320-3325

[62] Butzbach G. D., Wendorff J. H.: Polycarbonate-poly(methyl methacrylate) blends: the role of molecular interactions on miscibility and antiplasticization. Polymer 32 (1991), S. 7

[63] Agari Y., Ueda A.: Thermal diffusivity and conductivity of PMMA/PC blends. Polymer 38 (1997), S. 801-807

[64] Dixit M., Shaktawat V., Sharma K., Saxena N. S., Sharma T. P.: Mechanical Characterization of Polymethyl Methacrylate and Polycarbonate Blends. AIP Conference Proceedings 1004 (2008), S. 311

[65] Störmer M.: Struktur, Mikrostruktur und innere Spannungen laserdeponierter metallischer Legierungen. Dissertation Institut für Materialphysik, Universität Göttingen (1998)

[66] Wassermann H. J., Vermaak J. S.: On the determination of the surface stress of copper and platinum. Surface science 32 (1972), S. 168-174

[67] http://www.uni-saarland.de/fak7/hartmann/files/docs/pdf/ teaching/lectures/talks/WS0708/Cluster.pdf (18.07.2012, 12:18h)

[68] Meschede A., Krebs H.U.: Minimization of interface roughness in laser deposited $\mathrm{Fe} / \mathrm{MgO}$ multilayers. Appl. Phys. A 102 (2011), S. 137-140 
[69] Cammarata R.C., Trimble T.M.: Surface stress model for intrinsic stresses in thin films. J. Mater. Res. 15 (2000), S. 11

[70] Cammarata R.C.: Surface and interface stress effects on interfacial and nanostructured materials. Materials Science and Engineering A 237 (1997), S. 180-184

[71] Floro J. A., Hearne S. J., Hunter J. A., Kotula P.: The dynamic competition between stress generation and relaxation mechanisms during coalescence of VolmerWeber thin films. Journal of Applied Physics 89 (2001), S. 9

[72] Seel S. C., Thompson C. V., Hearne S. J., Floro J. A.: Tensile stress evolution during deposition of Volmer-Weber thin films. Journal of Applied Physics 88 (2000), S. 7079-7088

[73] ScharF T.: Mechanische Spannungen und Struktur laserdeponierter metallischer Filme in Abhängigkeit von der Teilchenenergie. Diplomarbeit Institut für Materialphysik, Universität Göttingen (2001)

[74] KNORR I.: Mechanische Eigenschaften kristallin-amorpher Schichtpakete. Dissertation Institut für Materialphysik, Universität Göttingen (2012)

[75] Krebs H.-U.: Charakteristische Eigenschaften laserdeponierter metallischer Systeme. Habilitationsschrift Fachbereich Physik, Universität Göttingen (1995)

[76] Dimitriadis E. K., Horkay F., Maresca J., Bechara K., Chadwick R. S.: Determination of Elastic Moduli of Thin Layers of Soft Material Using the Atomic Force Microscope. Biophysical Journal 82 (2002), S. 2798-2810

[77] Chizhik S. A., Huang Z., Gorbunov V. V., Myshrin N. K., Tsukruk V. V.: Micromechanical Properties of Elastic Polymeric Materials as Probed by Scanning Force Microscopy. Langmuir 14 (1998), S. 2606-2609

[78] Stafford C.M., Guo S., Harrison C., Chiang M.Y.M.: Combinatorial and highthroughput measurements of the modulus of thin polymer films. Review of scientific instruments 76 (2005), S. 062207

[79] Nolte A. J., Rubner M. F., Cohen R. E.: Determining the Young's Modulus of Polyelectrolyte Multilayer Films via Stress-Induced Mechanical Buckling Instabilities. Macromolecules 38 (2005), S. 5367-5370

[80] Sturm K.: Einfluß einer Argon Atmosphäre auf die gepulste Laserdeposition von Metallschichten. Dissertation Institut für Materialphysik, Universität Göttingen (2000) 
[81] Zailer I., Frost J. E. F., Chabasseur-Molyneux V., Ford C. J. B., Pepper M.: Crosslinked PMMA as a high-resolution negative resist for electron beam lithography and applications for physics of low-dimensional structures. Semicond. Sci. Technol. 11 (1996), S. 1235

[82] Westphal A.: Spannungsinduzierte Wellenbildung in laserdeponierten PMMA/PdDoppelschichten. Masterarbeit Institut für Materialphysik, Universität Göttingen (2013)

[83] Chua D. B. H., NG H. T.: Spontaneous formation of complex and ordered structures on oxygen-plasma-treated elastomeric polydimethylsiloxane. Applied physics letters 76 (2000), S. 6

[84] Y. Kim Y., Huang Q., Krishnaswamy S.: Selective growth and ordering of selfassembly on metal/polymer thin-film heterostructures via photothermal modulation. Applied Physics Letters 96 (2010), S. 123116

[85] Chung J. Y., Nolte A. J., Stafford C. M.: Diffusion-Controlled, Self-Organized Growth of Symmetric Wrinkling Patterns. Adv. Mater. 21 (2009), S. 1358-1362

[86] Mei H., Huang R., Chung J. Y., Stafford C. M., Yu H. H.: Buckling modes of elastic thin films on elastic substrates. Applied Physics Letters 90 (2007), S. 151902

[87] Goodberlet J. G.: Patterning 100 nm features using deep-ultraviolet contact photolithography. Appl Phys Lett. 76 (2000), S. 667

[88] Fuchs B., Schlenkrich F., Seyffarth S., Meschede A., Rotzoll R., Vana P., Grossmann P., Mann K., Krebs H.U.: Hardening of smooth pulsed laser deposited PMMA films by heating. Appl Phys A 98 (2010), S. 711-715

[89] Kobayashi H., Takahashi H., Hiki Y.: Viscosity measurement of organic glasses below and above glass transition temperature. Journal of Non-Crystalline Solids 290 (2001), S. $32-40$

[90] Demtroeder Prof. Dr. W.: Experimentalphysik 2, Elektrizität und Optik. Springer Verlag Berlin Heidelberg New York 3. Auflage

[91] RÖDER J.: Analyse der Glättung rauer Oberflächen durch Dünnschichtdeposition. Dissertation Institut für Materialphysik, Universität Göttingen (2009) 



\section{Danksagung}

Hiermit möchte ich mich zum Abschluss ganz herzlich bei all denen bedanken, die für das Gelingen dieser Arbeit beigetragen haben.

Ein ganz besonderer Dank gebührt Prof. Dr. Hans-Ulrich Krebs zum einen für die Aufnahme in seine Arbeitsgruppe und die exzellente Betreuung. Danke für die vielen gemeinsamen wissenschaftlichen Diskussionen, welche immer wieder zu neuen Denkanstößen geführt haben und somit diese Arbeit um ein Vielfaches bereichert haben. Zum anderen bedanke ich mich sehr für die Möglichkeit Wissenschaft und Familie stressfrei vereinen zu können.

Ein weiterer Dank geht an Prof. Dr. Samwer für die Zweitkorrektur dieser Arbeit.

Für die schöne Atmosphäre bedanke ich mich ganz herzlich bei der ganzen PLDArbeitsgruppe: Christian Eberl, Florian Döring, Anna Major, Sinja Pagel und Bea Jaquet. Danke für die vielen Motivationen, schönen Teerunden, gemeinsames Tüffteln am und im Laser und die stetige Unterstützung! Ich wünsche Euch viel Spaß und Erfolg für Eure Arbeiten. Ein großer Dank gilt auch allen Ehemaligen der Arbeitsgruppe: Tobias Liese, Sarah Hoffmann, Benedikt Ernst, Britta Fuchs, Andreas Meschede und Johanna Röder. Insbesondere Tobi danke ich für dieses einzigartige und exzellente Laserprogramm, aber auch für die vielen schönen Stunden im Büro. Für die tolle Zusammenarbeit bedanke ich mich bei Anja Westphal, Stephanie Demuth und Sara Lisa Jäckle.

Für die technische Unterstützung bedanke ich mich bei Conni Mewes, Michael Malchow und Dieter Plischke. Großer Dank geht auch an die Feinmechanikwerstatt und die Elektronik-Werkstatt für die großartige Hilfe und Unterstützung. Für die schöne Atmosphäre bedanke ich mich bei allen Mitarbeitern des Instituts!

Für die gute Zusammenarbeit hinsichtlich der mechanischen Untersuchungen bedanke ich mich ganz herzlich bei Prof. Dr. Volkert, Inga Knorr, Timo Wuttke und Julia Oelker. Bei Klaus Mann und Peter Großmann aus dem LaserLab bedanke ich mich für die NEXAFS-Untersuchungen. Für die SEC-Messungen bedanke ich mich bei Robert 
Rotzoll aus der Physikalischen Chemie. Matthias Büchsenschütz-Göbeler danke ich für die AFM-Indentermessungen.

Für die finanzielle Unterstützung bedanke ich mich bei der Deutschen Forschungsgemeinschaft, insbesondere durch den Sonderforschungsbereich 602 und den Graduiertenkolleg 782 .

Bei all meinen Freunden möchte ich mich für die schöne Zeit und die große Unterstützung bedanken.

Ein ganz großer Dank geht auch an meine Eltern und meinen Bruder. Danke, dass ihr immer für mich da seid und mich so gut unterstützt!

Lieber Felix, du bist mein Fels in der Brandung! Danke für die vielen aufbauenden Worte und das Gefühl immer jemanden an seiner Seite zu wissen. Meinem Sohn Henri danke ich für sein strahlendes Lachen, welches sofort alle Sorgen vergessen lässt. 


\section{Lebenslauf}

Angaben zur Person

Name:

Geburtsdatum:

Geburtsort:

Staatsangehörigkeit:

Familienstand:

\section{Schulbildung}

1990 - 1994

$1994-1996$

$1996-2003$

27.06 .2003

\section{Studium}

$10.2003-10.2005$

17.10.2005

$10.2005-06.2009$

12.12 .2008

01.2009
Susanne Schlenkrich, geb. Seyffarth

12.12.1983

Salzgitter

deutsch

verheiratet, ein Kind (31.08.2012)

Grundschule am Sonnenberg, Salzgitter

Orientierungsstufe am Sonnenberg, Salzgitter

Kranich-Gymnasium, Salzgitter

Allgemeine Hochschulreife

Grundstudium der Physik an der Georg-August-Universität Göttingen

Vordiplom in Physik

Hauptstudium der Physik an der Georg-August-Universität Göttingen

Diplom in Physik,

Thema der Diplomarbeit: „Epitaktisches Wachstum von $\mu \mathrm{m}-$ großen Kupferinseln auf Silizium", angefertigt am Institut für Materialphysik in der Arbeitsgruppe von Prof. Dr. H.-U. Krebs. Beginn der Promotion am Institut für Materialphysik in der Arbeitsgruppe von Prof. Dr. H.-U. Krebs,

wissenschaftliche Mitarbeiterin in den Sonderforschungsbereichen 602 „Komplexe Strukturen in kondensierter Materie“ 